\title{
WestVirginiaUniversity
}

THE RESEARCH REPOSITORY @ WVU

Graduate Theses, Dissertations, and Problem Reports

2020

\section{Weighted Modulo Orientations of Graphs}

Jianbing Liu

West Virginia University, jl0068@mix.wvu.edu

Follow this and additional works at: https://researchrepository.wvu.edu/etd

Part of the Discrete Mathematics and Combinatorics Commons

\section{Recommended Citation}

Liu, Jianbing, "Weighted Modulo Orientations of Graphs" (2020). Graduate Theses, Dissertations, and Problem Reports. 7757.

https://researchrepository.wvu.edu/etd/7757

This Thesis is protected by copyright and/or related rights. It has been brought to you by the The Research Repository @ WVU with permission from the rights-holder(s). You are free to use this Thesis in any way that is permitted by the copyright and related rights legislation that applies to your use. For other uses you must obtain permission from the rights-holder(s) directly, unless additional rights are indicated by a Creative Commons license in the record and/ or on the work itself. This Thesis has been accepted for inclusion in WVU Graduate Theses, Dissertations, and Problem Reports collection by an authorized administrator of The Research Repository @ WVU. For more information, please contact researchrepository@mail.wvu.edu. 


\title{
Weighted Modulo Orientations of Graphs
}

\author{
Jianbing Liu \\ Dissertation submitted to the \\ Eberly College of Arts and Sciences \\ at West Virginia University \\ in partial fulfillment of the requirements \\ for the degree of \\ Doctor of Philosophy \\ in \\ Mathematics

\begin{abstract}
Hong-Jian Lai, Ph.D., Chair
John Goldwasser, Ph.D.

K. Subramani (CSEE), Ph.D.

Rong Luo, Ph.D.

Jerzy Wojciechowski, Ph.D.

Department of Mathematics

West Virginia Univesity

Morgantown, West Virginia
\end{abstract} \\ 2020
}

Keywords: Nowhere-zero Flow, Group Connectivity, Modulo Orientation Graphic Sequence, Weighted Modulo Orientation, Additive Bases Matching Number, Signed Graph

Copyright 2020 Jianbing Liu 


\section{ABSTRACT \\ Weighted Modulo Orientations of Graphs}

\section{Jianbing Liu}

This dissertation focuses on the subject of nowhere-zero flow problems on graphs. Tutte's 5-Flow Conjecture (1954) states that every bridgeless graph admits a nowhere-zero 5-flow, and Tutte's 3-Flow Conjecture (1972) states that every 4-edge-connected graph admits a nowherezero 3-flow. Extending Tutte's flows conjectures, Jaeger's Circular Flow Conjecture (1981) says every $4 k$-edge-connected graph admits a modulo $(2 k+1)$-orientation, that is, an orientation such that the indegree is congruent to outdegree modulo $(2 k+1)$ at every vertex. Note that the $k=1$ case of Circular Flow Conjecture coincides with the 3-Flow Conjecture, and the case of $k=2$ implies the 5 -Flow Conjecture. This work is devoted to providing some partial results on these problems.

In Chapter 2, we study the problem of modulo 5-orientation for given multigraphic degree sequences. We prove that a multigraphic degree sequence $d=\left(d_{1}, \ldots, d_{n}\right)$ has a realization $G$ with a modulo 5-orientation if and only if $d_{i} \neq 1,3$ for each $i$. In addition, we show that every multigraphic sequence $d=\left(d_{1}, \ldots, d_{n}\right)$ with $\min _{1 \leq i \leq n} d_{i} \geq 9$ has a 9-edge-connected realization which admits a modulo 5-orientation for every possible boundary function. Jaeger conjectured that every 9-edge-connected multigraph admits a modulo 5-orientation, whose truth would imply Tutte's 5-Flow Conjecture. Consequently, this supports the conjecture of Jaeger.

In Chapter 3, we show that there are essentially finite many exceptions for graphs with bounded matching numbers not admitting any modulo $(2 k+1)$-orientations for any positive integers $t$. We additionally characterize all infinite many graphs with bounded matching numbers but without a nowhere-zero 3-flow. This partially supports Jaeger's Circular Flow Conjecture and Tutte's 3-Flow Conjecture.

In 2018, Esperet, De Verclos, Le and Thomass introduced the problem of weighted modulo orientations of graphs and indicated that this problem is closely related to modulo orientations of graphs, including Tutte's 3-Flow Conjecture. In Chapter 4 and Chapter 5, utilizing properties of additive bases and contractible configurations, we reduced the Esperet et al's edge-connectivity lower bound for some (signed) graphs families including planar graphs, complete graphs, chordal graphs, series-parallel graphs and bipartite graphs, indicating that much lower edge-connectivity bound still guarantees the existence of such orientations for those graph families.

In Chapter 6, we show that the assertion of Jaeger's Circular Flow Conjecture with $k=2$ holds asymptotically almost surely for random 9-regular graphs. 


\section{Acknowledgements}

First and foremost, I am most indebted to my supervisor, Dr. Hong-Jian Lai, for his continued encouragement and support over these last few years. It is a pleasure to work under his supervision. Without him, this dissertation could not have come about.

I would like to thank my other committee members: Dr. John Goldwasser, Dr. K. Subramani, Dr. Rong Luo and Dr. Jerzy Wojciechowski for their help during my studies. My thanks also goes to all the professors who have given me support and help in my study and in my daily life.

Finally, I would like to thank the Department of Mathematics and Eberly College of Arts and Sciences at West Virginia University for providing me with an excellent study environment and support during my study as a graduate student. 


\section{Contents}

1 Preliminaries $\quad 1$

1.1 Notation and Terminology . . . . . . . . . . . . . . . . . . . 1

1.2 Nowhere-zero Flow Problems . . . . . . . . . . . . . . . . . 2

1.3 Main Results . . . . . . . . . . . . . . . . . . . . 5

2 Modulo 5-Orientations and Degree Sequences $\quad 8$

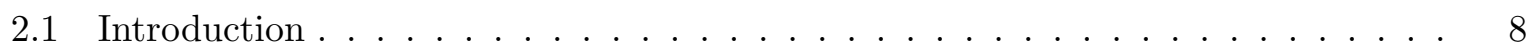

2.2 Proofs of Main Results . . . . . . . . . . . . . . . . . . . 11

2.2.1 Proof of Theorem $1.3 .2 \ldots \ldots \ldots \ldots \ldots \ldots$

2.2 .2 Proof of Theorem $1.3 .1 \ldots \ldots \ldots \ldots \ldots$

2.2 .3 Proof of Theorem $1.3 .3 \ldots \ldots \ldots \ldots \ldots$

3 Modulo Orientations and Matchings in Graphs 22

3.1 Introduction . . . . . . . . . . . . . . . . . . . 22

3.2 Modulo Orientations and Matchings . . . . . . . . . . . . . . . 26

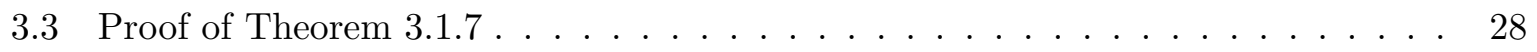

4 Weighted Modulo Orientations of Graphs $\quad 36$

4.1 Introduction . . . . . . . . . . . . . . . . . . 36

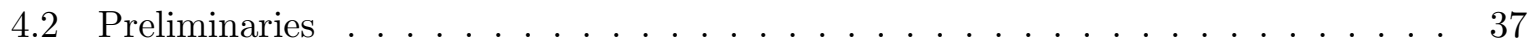

$4.2 .1 \quad$ Additive bases of $Z\left(G, \mathbb{Z}_{p}\right) \ldots \ldots \ldots \ldots \ldots \ldots$

4.2.2 A family of graphs admitting $(f, b ; p)$-orientations . . . . . . . . . 39

4.3 Proof of Theorem 1.3.9 . . . . . . . . . . . . . . . . . . . . 43

5 Weighted Modulo Orientations of Graphs and Signed Graphs 50

5.1 Introduction . . . . . . . . . . . . . . . . . 50

5.2 Weighted Modulo Orientations of Certain Graphs . . . . . . . . . . . . . . 52

5.2 .1 Complete Graphs . . . . . . . . . . . . . . . . . 52

5.2 .2 Chordal Graphs . . . . . . . . . . . . . . . . . 54 
5.2 .3 Series-parallel graphs . . . . . . . . . . . . . . . . . . 55

5.3 Complete Bipartite Graphs and Graphs with Small Matching Number . . . . . . 56

5.4 Signed graphs . . . . . . . . . . . . . . . . . . . . . . 60

6 Almost all 9-Regular Graphs admit a Modulo 5-Orientation $\quad 66$

6.1 Introduction . . . . . . . . . . . . . . . . . . . . 66

6.2 Proof of Theorem $6.1 .1 \ldots \ldots \ldots \ldots \ldots$

$\begin{array}{lll}7 & \text { Final Remarks } & \mathbf{7 3}\end{array}$

7.1 Validity of flow conjectures on Cayley graphs . . . . . . . . . . . . . . 73

7.2 Weighted modulo orientation of graphs . . . . . . . . . . . . . . . 74 


\section{Chapter 1}

\section{Preliminaries}

\subsection{Notation and Terminology}

The graphs in this paper are finite and loopless, and may contain parallel edges. We follow [8] for undefined terms and notations. For a graph $G$, let $\alpha^{\prime}(G), \kappa^{\prime}(G)$ and $\delta(G)$ denote the matching number, the edge-connectivity, and the minimum degree of $G$, respectively. If two vertices $u$ and $v$ are adjacent in $G$, then write $u \sim v$. For a vertex $z \in V(G)$, define $N_{G}(z)=$ $\{v \in V(G): z v \in E(G)\}$ to be the neighborhood of $z$. For vertex subsets $U, W \subseteq V(G)$, let $[U, W]_{G}=\{u w \in E(G) \mid u \in U, w \in W\}$. When $U=\{u\}$ or $W=\{w\}$, we use $[u, W]_{G}$ or $[U, w]_{G}$ for $[U, W]_{G}$, respectively. We also use $\partial_{G}(S)=[S, V(G)-S]_{G}$ to denote an edge-cut of $G$. Let $D=D(G)$ be an orientation of $G$. We denote $(u, w)$ to be an arc oriented from $u$ to $w$, and $A(D)$ to be the set of all $\operatorname{arcs}$ in $D$. Define $[U, W]_{D}=\{(u, w) \in A(D): u \in U, w \in W\}$. For notational convenience, we denote $E_{D}^{+}(v)=[\{v\}, V(D)-\{v\}]_{D}$ and $E_{D}^{-}(v)=[V(D)-\{v\},\{v\}]_{D}$ for a vertex $v \in V(D)$, respectively. When $W=V(G)-U$, we also denote $\delta_{D}^{+}(W)=[W, U]_{D}$, $\partial_{G}(W)=[W, U]_{G}$ and $d_{G}(W)=\left|\partial_{G}(W)\right|$. In addition, $d_{G}(v)=\left|E_{G}(v)\right|, d_{D}^{-}(v)=\left|E_{D}^{-}(v)\right|$ and $d_{D}^{+}(v)=\left|E_{D}^{+}(v)\right|$ are known as the degree, indegree and outdegree of $v$, respectively. The subscript may be omitted if it is understood from the context. For a graph $G$ and integer $k>0$, $k G$ denotes the graph obtained from $G$ by replacing each edge with $k$ parallel edges joining the same pair of vertices. For an edge set $X \subseteq E(G)$, the contraction $G / X$ is the graph obtained from $G$ by identifying the two ends of each edge in $X$, and then deleting the resulting loops. If $H$ is a subgraph of $G$, then we use $G / H$ for $G / E(H)$. For a vertex set $W \subset V(G)$ such that $G[W]$ is connected, we also use $G / W$ for $G / G[W]$. 


\subsection{Nowhere-zero Flow Problems}

The concept of integer flow was introduced by Tutte $[85,86]$ as a generalization of map-coloring problems. Let $\Gamma$ be an Abelian group, let $D$ be an orientation of $G$ and $f: E(G) \rightarrow \Gamma$. The pair $(D, f)$ is $\Gamma$-flow in $G$ if the following condition is satisfied at every vertex $v \in V(G)$ :

$$
\sum_{e \in E_{D}^{+}(v)} f(e)=\sum_{e \in E_{D}^{-}(v)} f(e)
$$

An integer flow $(D, f)$ is called a nowhere-zero $k$-flow (or $k$-NZF) if $1 \leq|f(e)| \leq k-1$, for each edge $e \in E(G)$. A graph is bridgeless if it is 2-edge-connected, and bridgeless is a necessary condition for a graph to admit a nowhere-zero flow. Tutte established the relation between face-coloring problems and integer flows, which motivates the study of the theory of integer flow.

Theorem 1.2.1. (Tutte [86]) Let $G$ be a bridgeless plane graph. Then $G$ is $k$-face-colorable if and only if $G$ admits a nowhere-zero $k$-flow.

Thus, the Four Color Problem of planar graph that every plane graph is 4-face-colorable is essentially equivalent to the statement : every bridgeless planar graph admits a nowhere-zero 4flow. This motivated Tutte $[80,86]$ to propose the most fascinating conjectures in graph theory, which generalize three theorems on planar graphs, the Five-Color Theorem, the Four-Color Theorem (a conjecture at that time) and Grötzsch's Three-Color Theorem.

Conjecture 1.2.1. (Tutte's flow conjectures)

(1) (5-Flow Conjecture, 1954) Every bridgeless graph admits a nowhere-zero 5-flow.

(2) (4-Flow Conjecture, 1966) Every bridgeless graph without Petersen-minor admits a nowherezero 4-flow.

(3) (3-Flow Conjecture, 1972) Every 4-edge-connected graph admits a nowhere-zero 3-flow.

The Four Color Problem was solved by Appel and Haken [1,2], see also [70], known as the Four Color Theorem. Tutte's flow conjectures remain open so far to the best of our knowledge.

As observed by Tutte [86], the existence of nowhere-zero $k$-flows is equivalent to the existence of nowhere-zero $\mathbb{Z}_{k}$-flows. The study of integer flows focuses more on nowhere-zero $\mathbb{Z}_{k}$-flows as it is easier to handle. Motivated by this fact, Jaeger further generalized integer flow theory to introduce the concept of group connectivity and modulo orientation.

A graph is $\mathbb{Z}_{k}$-connected if $G$ has an orientation $D(G)$ such that for every function $b$ : $V(G) \rightarrow \mathbb{Z}_{k}$ with $\sum b(v) \equiv 0(\bmod k)$, there exists a mapping $f: E(G) \mapsto \mathbb{Z}_{k}-\{0\}$ with

$$
\sum_{e \in E_{D}^{+}(v)} f(e)-\sum_{e \in E_{D}^{-}(v)} f(e) \equiv b(v) \quad(\bmod k), \text { for every vertex } v \in V(G) .
$$


As in [8], $d_{D}^{+}(v)=\left|E_{D}^{+}(v)\right|$ and $d_{D}^{-}(v)=\left|E_{D}^{-}(v)\right|$ denote the out-degree and the in-degree of $v$ under the orientation $D$, respectively. If a graph $G$ has an orientation $D$ such that $d_{D}^{+}(v) \equiv d_{D}^{-}(v)(\bmod k)$ for every vertex $v \in V(G)$, then we say that $G$ admits a modulo $k$-orientation. Let $\mathcal{M}_{k}$ denote the family of all graphs with a modulo $k$-orientation. Note that the modulo $k$-orientation differs dramatically for $k$ with different parity. For even $k$, modulo $k$-orientation is obtained for even graphs only, while modulo $k$-orientation for odd $k$ is a NPcomplete problem. The concept of strongly $\mathbb{Z}_{k}$-connectedness was introduced in $[42,44]$, serving as contractible configurations for modulo orientations. A graph $G$ is strongly $\mathbb{Z}_{k}$-connected if for every $b: V(G) \rightarrow \mathbb{Z}_{k}$ with $\sum_{v \in V(G)} b(v) \equiv 0(\bmod k)$, there is an orientation $D$ such that $d_{D}^{+}(v)-d_{D}^{-}(v) \equiv b(v)(\bmod k)$ for every vertex $v \in V(G)$. Let $\left\langle\mathcal{S} \mathbb{Z}_{k}\right\rangle$ denote the family of all strongly $\mathbb{Z}_{k}$-connected graphs. For a nonnegative integer $k, \mathbb{Z}_{k}$-connected graphs and strongly $\mathbb{Z}_{2 k+1}$-connected graphs are contractible configurations for nowhere-zero $k$-flows and modulo $(2 k+1)$-orientations, respectively. Jaeger proposed the following conjectures concerning group connectivity and modulo $(2 k+1)$-orientations.

Conjecture 1.2.2. (Jaeger's Circular Flow Conjecture, [34]) Every 4k-edge-connected graph admits a modulo $(2 k+1)$-orientation.

Conjecture 1.2.3. (1) (Jaeger et al., [36]) Every 3-edge-connected graph is $\mathbb{Z}_{5}$-connected.

(2) (Jaeger et al., [36]) Every 5-edge-connected graph is $\mathbb{Z}_{3}$-connected.

(3) (Lai, [42]) Every $(4 k+1)$-edge-connected graph is strongly $\mathbb{Z}_{2 k+1}$-connected.

Note that Conjecture 1.2.3 (1)-(3) are strengthen of Conjecture 1.2.1 and Conjecture 1.2.2 on group connectivity. For $k=1$, Conjecture 1.2.2 is Conjecture 1.2.1 (3) (3-Flow Conjecture). By Jaeger [39], the case of $k=2$ in Conjecture 1.2.2, if true, would imply Conjecture 1.2.1 (1) (5-Flow Conjecture). However, it was disproved for all $k \geq 3$ in [31]. It was observed by Jaeger [35] that if the graph $3 G$ has a modulo 5-orientation, then $G$ admits a nowhere-zero $\mathbb{Z}_{5}$-flow. This led Jaeger [35] to propose the following stronger conjecture, whose truth implies Tutte's 5-flow conjecture.

Conjecture 1.2.4. (Jaeger [35]) Every 9-edge-connected multigraph admits a modulo 5-orientation.

Conjecture 1.2.4 is further strengthened to the following conjecture in Conjecture 1.2.3 (3) for $k=2$.

Conjecture 1.2.5. (Lai [42]) Every 9-edge-connected multigraph is strongly $\mathbb{Z}_{5}$-connected.

Conjectures 1.2.4 and 1.2.5 are confirmed for 12-edge-connected multigraphs by Lov́asz, Thomassen, Wu and Zhang [60]. We also note that, by a result in [47], the truth of Conjecture 1.2.5 would imply Conjecture 1.2.3 (1).

Some of the best known results are the following. 
Theorem 1.2.2. (8-Flow Theorem, Jaeger [33], Kilpatrick [37]) Every bridgeless graph admits a nowhere-zero 8-flow.

Seymour [76] improved the 8-Flow Theorem to get the 6-Flow Theorem which remains the strongest partial result to the 5-Flow Conjecture.

Theorem 1.2.3. (6-Flow Theorem, Seymour, [76]) Every bridgeless graph admits a nowherezero 6 -flow.

Jaeger [33] also got the following result concerning to 4-Flow Theorem.

Theorem 1.2.4. (Jaeger [33]) Every 4-edge-connected graph admits a nowhere-zero 4-flow.

The best approach to Conjecture 1.2.1 (2) (4-Flow Conjecture) is known as the snark theorem recently proved by Robertson, Sanders, Seymour and Thomas, see [22,71-74]

Theorem 1.2.5. (Snark Theorem, Edwards, Robertson, Sanders, Seymour and Thomas, 2000s ) Every bridgeless cubic graph without Petersen-minor admits a nowhere-zero 4-flow.

Some early partial results on the 3-Flow Conjecture can be found in [59] and [4]. Thomassen proved the following strengthened theorem.

Theorem 1.2.6. (Thomassen, [77]) Every 8-edge-connected graph is $\mathbb{Z}_{3}$-connected.

Thomassen's result was further improved by Lovász, Thomassen, Wu and Zhang. In particular, Theorem 1.2.7 indicates that every 6-edge-connected graph is $\mathbb{Z}_{3}$-connected, and therefore admits a nowhere-zero 3-flow.

Theorem 1.2.7. (Lovász, Thomassen, Wu and Zhang [60]) Let $k>0$ be an integer. Every $6 k$-edge-connected graph $G$ has a b-orientation for every $\mathbb{Z}_{2 k+1}$-boundary $b$ of $G$.

Theorem 1.2.8. (Han, Li, Wu and Zhang [31], Li [48]) Let $k>0$ be an integer.

(i) If $k \geq 3$, then there exists a $4 k$-edge-connected graph admitting no modulo $(2 k+1)$-orientation.

(i) If $k \geq 5$, then there exists a $(4 k+1)$-edge-connected graph admitting no modulo $(2 k+1)$ orientation.

Note that Theorem 1.2.8 disproved Jaeger's Circular Flow Conjecture, which Jaeger [35] conjectured that every $4 k$-edge-connected graph admits a modulo $(2 k+1)$-orientation. Further expository of the problem can be found in the informative monograph by Zhang [87].

Aiming at extending Theorem 1.2.7, Esperet et al in [23] defined a modulo $k f$-weighted $b$-orientation of a graph $G$, for a given mapping $f \in F\left(G, \mathbb{Z}_{k}\right)$ and a $\mathbb{Z}_{k}$-boundary $b$, to be an orientation $D=D(G)$ satisfying $\partial_{D}(f) \equiv b(\bmod k)$ under $D$. Throughout the rest of the thesis, we shall abbreviate a modulo $k f$-weighted $b$-orientation as an $(f, b ; k)$-orientation. Esperet et al indicated in [23] that to investigate $(f, b ; k)$-orientation of graphs, it is necessary to assume that $k$ to be an odd prime number. The following is proved in [23]. 
Theorem 1.2.9. (Esperet, De Verclos, Le and Thomassé, [23]) Let $p \geq 3$ be a prime number and $G$ be a $\left(6 p^{2}-14 p+8\right)$-edge-connected graph. Then for any mapping $f \in F\left(G, \mathbb{Z}_{p}^{*}\right)$ and any $\mathbb{Z}_{p}$-boundary $b$ of $G, G$ has an $(f, b ; p)$-orientation.

\subsection{Main Results}

Motivated by Conjectures 1.2.1-1.2.4 and Theorems 1.2.7-1.2.9, one of our study is to verify some of these conjectures under the specific conditions and the other is to investigate the relationship between the edge-connectivity of a graph and its $(f, b ; p)$-orientability over the finite prime field $\mathbb{Z}_{p}$ for any mapping $f \in F\left(G, \mathbb{Z}_{p}^{*}\right)$ and any $\mathbb{Z}_{p}$-boundary $b$ of $G$.

we study the degree sequences with realizations that are strongly $\mathbb{Z}_{5}$-connected or have modulo 5-orientation properties. Our main results are the following characterizations.

Theorem 1.3.1. ( [32]) For any multigraphic sequence $d=\left(d_{1}, d_{2}, \cdots, d_{n}\right)$, $d$ has a modulo 5 -orientation realization if and only if $d_{i} \notin\{1,3\}$ for every $1 \leq i \leq n$.

Theorem 1.3.2. ( [32]) For any multigraphic sequence $d=\left(d_{1}, d_{2}, \cdots, d_{n}\right)$, d has a strongly $\mathbb{Z}_{5}$-connected realization if and only if $\sum_{i=1}^{n} d_{i} \geq 8 n-8$ and $\min _{i \in[n]} d_{i} \geq 4$.

In addition, we obtain the following theorem, which provides partial evidences for Conjecture 1.2.3 (3) with $p=2$ and Conjecture 1.2.4.

Theorem 1.3.3. ( [32]) For any multigraphic sequence $d=\left(d_{1}, d_{2}, \cdots, d_{n}\right)$ with $\min _{i \in[n]} d_{i} \geq 9$, $d$ has a 9-edge-connected strongly $\mathbb{Z}_{5}$-connected realization.

Theorem 1.3.3 also leads to the following corollary.

Corollary 1.3.4. ( [32]) For any multigraphic sequence $d=\left(d_{1}, d_{2}, \cdots, d_{n}\right)$ with $\min _{i \in[n]} d_{i} \geq 8$, $d$ has a 8-edge-connected modulo 5-orientation realization.

We show that if a family of graphs has bounded matching number, then after certain reduction operation, there are only finitely many $(2 k+2)$-edge-connected graphs without modulo $(2 k+1)$-orientation in this family. Our first main result can be formally restated as follows.

Theorem 1.3.5. ( [55]) For any integer $s>0$, there exists a finite graph family $\mathcal{G}(k, s)$ such that, for every graph $G$ with $\kappa^{\prime}(G) \geq 2 k+2$ and $\alpha^{\prime}(G) \leq s, G$ has a modulo $(2 k+1)$-orientation if and only if its $\left\langle\mathcal{S} \mathbb{Z}_{2 k+1}\right\rangle$-reduction is not in $\mathcal{G}(k, s)$.

Theorem 5.3.4, together with Theorem 1.2.7, immediately implies the following ChvátalErdős type theorem: if $G$ satisfies a Chvátal-Erdős type condition $\kappa^{\prime}(G) \geq \max \left\{\alpha^{\prime}(G), 2 k+2\right\}$, then $G$ admits a modulo $(2 k+1)$-orientation with essentially finitely many exceptions. 
Theorem 1.3.6. ( [55]) For any integer $k>0$, there exists a finite family of non-modulo $(2 k+1)$ orientation admissible graphs $\mathcal{F}_{1}(k)$ such that, for every graph $G$ with $\kappa^{\prime}(G) \geq \max \left\{\alpha^{\prime}(G)\right.$, $2 k+2\}, G$ admits a modulo $(2 k+1)$-orientation if and only if $G$ cannot be contracted to a member in $\mathcal{F}_{1}(k)$.

Theorems 5.3.4 and 3.1.5 are the best possible in the sense that the condition of edge connectivity $2 k+2$ cannot be replaced by $2 k+1$. In fact, there are infinitely many $(2 k+1)$-edgeconnected $\left\langle\mathcal{S} \mathbb{Z}_{2 k+1}\right\rangle$-reduced graphs with fixed matching number admitting no modulo $(2 k+1)$ orientation. Denote $K_{s, t}^{+k}$ to the graph obtained from the complete bipartite graph $K_{s, t}$ by adding $k$ new edges connecting vertices of degree $t$. We write $K_{s, t}^{+}$as an abbreviation of $K_{3, t}^{+1}$ in this paper. For example, let $\mathcal{K}(k)$ be a graph family defined by $\mathcal{K}(k)=\left\{K_{2 k+1, c}^{+}: c \geq 2 k+1\right\}$, where $K_{2 k+1, c}^{+}$denotes the graph obtained from the complete bipartite graph $K_{2 k+1, c}$ by adding a new edge connecting two degree $c$ vertices. Then each member in $\mathcal{K}(k)$ is a $(2 k+1)$-edge-connected $\left\langle\mathcal{S} \mathbb{Z}_{2 k+1}\right\rangle$-reduced graph without a modulo $(2 k+1)$-orientation.

On the other hand, for the modulo 3-orientation case, we improve Theorem 3.1.5 by releasing the edge connectivity condition and characterizing all the $\left\langle\mathcal{S} \mathbb{Z}_{3}\right\rangle$-reduced graph in this family.

Theorem 1.3.7. ( [55]) Let $G$ be a bridgeless graph with $\kappa^{\prime}(G) \geq \alpha^{\prime}(G)$. Then $G$ admits a nowhere-zero 3-flow, unless $G$ belongs to one of the exceptional cases.

In particular, Theorem 3.1.7 verifies Tutte's 3-Flow Conjecture for graphs with $\kappa^{\prime}(G) \geq$ $\alpha^{\prime}(G)$. Kochol [39] showed that the 3-Flow Conjecture is equivalent to a seemly stronger form that every bridgeless graph with at most three 3 -edge-cut admits a nowhere-zero 3 -flow. Theorem 3.1.7 also verifies this stronger statement for graphs with $\kappa^{\prime}(G) \geq \alpha^{\prime}(G)$.

Corollary 1.3.8. ( [55]) Let $G$ be a bridgeless graph $G$ with $\kappa^{\prime}(G) \geq \alpha^{\prime}(G)$. Then $G$ admits a nowhere-zero 3-flow provided that $G$ has at most three 3-edge-cut.

In the following, We investigate the relationship between the edge-connectivity of a graph embedded on a 2-manifold and its $(f, b ; p)$-orientability over the finite field $\mathbb{Z}_{p}$.

Theorem 1.3.9. ( [56]) Let $p>0$ be an odd prime, and let $G$ be a graph with Euler genus $g$ and edge connectivity

$$
\kappa^{\prime}(G) \geq \begin{cases}4 p-6+\lfloor g / 2\rfloor & \text { if } g \leq 2, \\ (p-2)\lfloor\sqrt{6 g+0.25}+2.5\rfloor+1 & \text { if } g \geq 3 \\ p \sqrt{4.98 g} & \text { if } g \text { is sufficiently large. }\end{cases}
$$

Then for any mapping $f \in F\left(G, \mathbb{Z}_{p}^{*}\right)$ and any $\mathbb{Z}_{p}$-boundary b of $G$, the graph $G$ has an $(f, b ; p)$ orientation. 
This result implies every planar graph with edge-connectivity $4 p-6$ admits an $(f, b ; p)$ orientation. Let $\mathcal{O}_{p}$ be a graph family in which admits an an $(f, b ; p)$-orientation. We also reduced Esperet et al's edge-connectivity lower bound of complete graphs, complete bipartite graphs and chordal graphs in $\mathcal{O}_{p}$.

Theorem 1.3.10. ( [57]) Let $K_{n}$ be a complete graph. If $n \geq 2(p-1)(5+3 \log (p-1))$, then $K_{n} \in \mathcal{O}_{p}$.

Theorem 1.3.11. ( [57]) Let $G$ be a connected chordal graph. If $\kappa(G) \geq 2(p-1)(5+3 \log (p-$ 1)) - 1 , then $G \in \mathcal{O}_{p}$.

Theorem 1.3.12. ( [57]) Let $p$ be an odd prime and $G$ be a complete bipartite graph $K_{n_{1}, n_{2}}$ with $n_{1}=\frac{1}{2}(p-1)(p-2)+1, n_{2}=\frac{1}{2} n_{1}\left(n_{1}-1\right)(p-1)$. For any mapping $f \in F\left(G, \mathbb{Z}_{p}^{*}\right)$ and any $\mathbb{Z}_{p}$-boundary $b$ of $G, G$ has an $(f, b ; p)$-orientation.

A signed graph is an ordered pair $(G, \sigma)$ consisting of a graph $G$ with a mapping $\sigma$ : $E(G) \rightarrow\{1,-1\}$. Let $\tau$ be an orientation of $(G, \sigma)$. For each vertex $v \in V(G)$, let $H_{G}(v)$ be the set of half edges incident with $v$. Define $\tau(h)=1$ if the half edge $h \in H_{G}(v)$ is oriented away from $v$, and $\tau(h)=-1$ if the half edge $h \in H_{G}(v)$ is oriented towards $v$. Let $(G, \sigma)$ be a 2-unbalanced signed graph. A mapping $b: V(G) \rightarrow \mathbb{Z}_{k}$ with $\sum_{v \in V(G)} b(v) \in 2 \mathbb{Z}_{k}$ and every $f: E(G) \rightarrow \mathbb{Z}_{k}^{*}$, an orientation $\tau$ of $(G, \sigma)$ is called an $(f, b ; k)$-orientation if for every vertex $v \in V(G)$

$$
\partial f(v)=\sum_{h \in H_{G}(v)} \tau(v) f\left(e_{h}\right)=b(v) .
$$

We showed the following theorem.

Theorem 1.3.13. ( [57]) Let $p$ be an odd prime and let $(G, \sigma)$ be a $(p-1)$-unbalanced signed graph with $\kappa^{\prime}(G) \geq 12 p^{2}-28 p+15$. Then $(G, \sigma)$ admits an $(f, b ; k)$-orientation. 


\section{Chapter 2}

\section{Modulo 5-Orientations and Degree Sequences}

This chapter includes joint work with Han and Lai, appeared in [32].

\subsection{Introduction}

Conjectures 1.2.4 and 1.2.5 are confirmed for 12-edge-connected multigraphs by Lov́asz, Thomassen, $\mathrm{Wu}$ and Zhang [60]. We also note that, by a result in [47], the truth of Conjecture 1.2.5 would imply another conjecture of Jaeger et al. [36] which states that every 3-edge-connected graph is $\mathbb{Z}_{5}$-connected. Denote $\left\langle\mathbb{Z}_{5}\right\rangle$ to be the family of all $\mathbb{Z}_{5}$-connected graphs.

An integral degree sequence $d=\left(d_{1}, d_{2}, \cdots, d_{n}\right)$ is called graphic (multigraphic, resp.) if there is a simple graph (multigraph, resp.) $G$ so that the degree sequence of $G$ equals $d$; such a graph $G$ is called a realization of $d$. Graphic and multigraphic sequences with certain flow and group connectivity properties have been extensively studied [18,47,61,62,83,84]. Specifically, all graphic sequences with nowhere-zero 3-flow or 4-flow realization are characterized by Luo et al. [61] [62], respectively. The problem of characterizing all degree sequences with $\mathbb{Z}_{3}$-connected properties is proposed and studied by Yang et al. [84], and solved by Dai and Ying [18]. In general, the $\mathbb{Z}_{k}$-connected realization problem is characterized for $k=4$ by Wu et al. [83], and it is eventually resolved in [47] for every $k$.

In this chapter, we study the degree sequences with realizations that are strongly $\mathbb{Z}_{5^{-}}$ connected or have modulo 5 -orientation properties. As $K_{1}$ is strongly $\mathbb{Z}_{5}$-connected, for any graph $G$, every vertex lies in a maximal strongly $\mathbb{Z}_{5}$-connected subgraph. Let $H_{1}, H_{2}, \cdots, H_{c}$ denote the collection of all maximal subgraphs in the graph $G$. Then $G^{\prime}=G /\left(\cup_{i=1}^{c} E\left(H_{i}\right)\right)$ is called the $\left\langle\mathcal{S} \mathbb{Z}_{5}\right\rangle$-reduction of $G$. If $G$ is strongly $\mathbb{Z}_{5}$-connected, then its $\left\langle\mathcal{S} \mathbb{Z}_{5}\right\rangle$-reduction is $K_{1}$, a singleton. 
The following lemma is a summary of some basic properties stated in [41], [42] and [44].

Lemma 2.1.1. ( $[41,42,44])$ Each of the following holds.

(i) If $H \in\left\langle\mathbb{Z}_{5}\right\rangle$ and $G / H \in\left\langle\mathbb{Z}_{5}\right\rangle$, then $G \in\left\langle\mathbb{Z}_{5}\right\rangle$.

(ii) A cycle of length $n$ is in $\left\langle\mathbb{Z}_{5}\right\rangle$ if and only if $n \leq 4$.

(iii) Let $m K_{2}$ denote the loopless graph with two vertices and $m$ parallel edges. Then $m K_{2}$ is strongly $\mathbb{Z}_{5}$-connected if and only if $m \geq 4$.

(iv) $G \in \mathcal{M}_{5}$ if and only if its $\left\langle\mathcal{S} \mathbb{Z}_{5}\right\rangle$-reduction $G^{\prime} \in \mathcal{M}_{5}$.

(v) $G \in\left\langle\mathcal{S} \mathbb{Z}_{5}\right\rangle$ if and only if its $\left\langle\mathcal{S} \mathbb{Z}_{5}\right\rangle$-reduction $G^{\prime}=K_{1}$.

The following theorem is a special case of the results stated in [47].

Theorem 2.1.2. [47] Let $G$ be a graph. Then each of the following holds.

(i) $G \in\left\langle\mathbb{Z}_{5}\right\rangle$ if and only if $3 G \in\left\langle\mathcal{S} \mathbb{Z}_{5}\right\rangle$.

(ii) If $G \in\left\langle\mathcal{S} \mathbb{Z}_{5}\right\rangle$, then $G$ contains four edge-disjoint spanning trees, and in particular, $|E(G)| \geq 4|V(G)|-4$.

For a realization $G$ of a multigraphic degree sequence $d=\left(d_{1}, d_{2}, \cdots, d_{n}\right)$, if $G$ is a realization of $d$ with $V(G)=\left\{v_{1}, \ldots, v_{n}\right\}$ such that $d_{G}\left(v_{i}\right)=d_{i}$, then $v_{i}$ is called the $d_{i}$-vertex for each $i \in[n]$. As a rearrangement of a degree sequence does not change its realizations, we will just focus on nonincreasing multigraphic sequence in the rest of the paper for convenience.

Theorem 2.1.3. (Hakimi [30]) Let $d=\left(d_{1}, d_{2}, \cdots, d_{n}\right)$ be a nonincreasing integral sequence with $n \geq 2$ and $d_{n} \geq 0$. Then $d$ is a multigraphic sequence if and only if $\sum_{i=1}^{n} d_{i}$ is even and $d_{1} \leq d_{2}+\cdots+d_{n}$.

Theorem 2.1.4. (Boesch and Harary [10]) Let $d=\left(d_{1}, \cdots, d_{n}\right)$ be a nonincreasing integral sequence with $n \geq 2$ and $d_{n} \geq 0$. Let $j$ be an integer with $2 \leq j \leq n$ such that $d_{j} \geq 1$. Then the sequence $\left(d_{1}, d_{2}, \cdots, d_{n}\right)$ is multigraphic if and only if the sequence $\left(d_{1}-1, d_{2}, \cdots, d_{j-1}, d_{j}-\right.$ $\left.1, d_{j+1}, \cdots, d_{n}\right)$ is multigraphic.

Let $G$ be a graph with $u v \in E(G)$ and let $w$ be a vertex different from $u$ and $v$, where $w$ may or maynot be in $V(G)$. Define $G^{(w, u v)}$ to be the graph containing $w$ obtained from $G-u v$ by adding new edges $w u$ and $w v$. We also say that $G^{(w, u v)}$ is obtained from $G$ by inserting the edge $u v$ to $w$ in this paper. The following observation is straightforward, which indicates the reverse operation of vertex splitting would preserve the edge connectivity.

Lemma 2.1.5. Let $G$ be a connected graph.

(i) Let $w \in V(G) \backslash\{u, v\}$ and $G^{\prime}=G^{(w, u v)}$. Then $\kappa^{\prime}\left(G^{\prime}\right) \geq \kappa^{\prime}(G)$.

(ii) Let $w \notin V(G)$ be a new vertex and $e_{1}, \cdots, e_{t} \in E(G)$. Then the graph $G^{\prime}$ obtained from $G$ by inserting the edges $e_{1}, \cdots, e_{t}$ to $w$ satisfies $\kappa^{\prime}\left(G^{\prime}\right) \geq \min \left\{\kappa^{\prime}(G), 2 t\right\}$. 
Proof. (i) Let $\left[X, X^{c}\right]_{G^{\prime}}$ be an edge cut of $G^{\prime}$. Observe that either $\left|\left[X, X^{c}\right]_{G^{\prime}}\right|=\left|\left[X, X^{c}\right]_{G}\right|$ or $\left|\left[X, X^{c}\right]_{G^{\prime}}\right|=\left|\left[X, X^{c}\right]_{G}\right|+2$ depending on the position of $u, v, w$ in $X$ or $X^{c}$. So $\left|\left[X, X^{c}\right]_{G^{\prime}}\right| \geq$ $\left|\left[X, X^{c}\right]_{G}\right| \geq \kappa^{\prime}(G)$, and thus $\kappa^{\prime}\left(G^{\prime}\right) \geq \kappa^{\prime}(G)$.

(ii) The proof of (ii) is similar to (i).

Let $x_{1} x_{2}, x_{2} x_{3} \in E(G)$. Denote $G_{\left[x_{2}, x_{1} x_{3}\right]}$ to be the graph obtained from $G-\left\{x_{1} x_{2}, x_{2} x_{3}\right\}$ by adding a new edge $x_{1} x_{3}$. The operation to get $G_{\left[x_{2}, x_{1} x_{3}\right]}$ from $G$ is referred as to lift the edges $x_{1} x_{2}, x_{2} x_{3}$ in $G$. The next lemma follows from the definition of strongly $\mathbb{Z}_{5}$-connectedness.

Lemma 2.1.6. Let $x_{1}, x_{2}, x_{3}$ and $G_{\left[x_{2}, x_{1} x_{3}\right]}$ be the same notation as defined above. If $G_{\left[x_{2}, x_{1} x_{3}\right]} \in$ $\left\langle\mathcal{S} \mathbb{Z}_{5}\right\rangle$, then $G \in\left\langle\mathcal{S} \mathbb{Z}_{5}\right\rangle$.
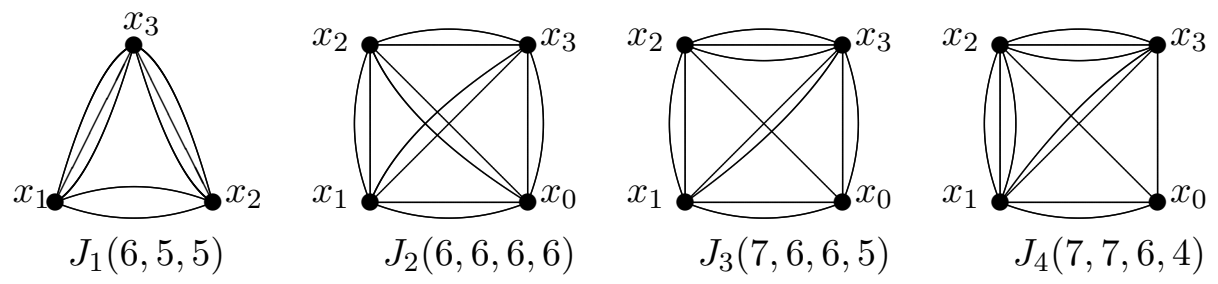

Figure 2.1: The graphs in Lemma 2.1.7.

The next lemma shows that the small graphs depicted in Figure 1 could play a crucial role in the inductive arguments of our proofs.

Lemma 2.1.7. Each of the graphs $J_{1}, J_{2}, J_{3}, J_{4}$ in Figure 1 is strongly $\mathbb{Z}_{5}$-connected.

Proof. (i) Let $b \in Z\left(J_{1}, \mathbb{Z}_{5}\right)$. If $b\left(x_{1}\right) \neq 0$, we lift two edges $x_{3} x_{1}, x_{1} x_{2}$ in $J_{1}$ to obtain the graph $J_{1\left[x_{1}, x_{2} x_{3}\right]}$. Since $\left|\left[x_{1},\left\{x_{2}, x_{3}\right\}\right]_{J_{1\left[x_{1}, x_{2} x_{3}\right]}}\right|=3$ and $b\left(x_{1}\right) \neq 0$, we can modify the boundary $b\left(x_{1}\right)$ with the three edges in $\left[x_{1},\left\{x_{2}, x_{3}\right\}\right]_{J_{\left[x_{1}, x_{2} x_{3}\right]}}$. Specifically, orient $2,0,3,1$ edges towards

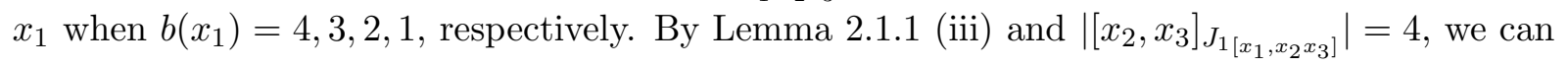
also modify the boundaries $b\left(x_{2}\right), b\left(x_{3}\right)$ with four parallel edges $x_{2} x_{3}$. By symmetry, we assume $b\left(x_{1}\right)=b\left(x_{2}\right)=0$, then $b\left(x_{3}\right)=0$ since $b \in Z\left(J_{1}, \mathbb{Z}_{5}\right)$. Orient all the edges in $\left[x_{1},\left\{x_{2}, x_{3}\right\}\right]_{J_{1}}$ towards $x_{1}$ and orient all the edges in $\left[x_{2},\left\{x_{1}, x_{3}\right\}\right]_{J_{1}}$ from $x_{2}$ to obtain an orientation of $J_{1}$, which agrees with the boundary $b\left(x_{1}\right)=b\left(x_{2}\right)=b\left(x_{3}\right)=0$. Therefore $J_{1}$ is strongly $\mathbb{Z}_{5}$-connected by definition.

(ii) Let $b \in Z\left(J_{2}, \mathbb{Z}_{5}\right)$. If $b\left(x_{0}\right)=0$, we lift three pairs of edges $\left\{x_{2} x_{0}, x_{0} x_{3}\right\},\left\{x_{2} x_{0}, x_{0} x_{1}\right\}$ and $\left\{x_{3} x_{0}, x_{0} x_{1}\right\}$ from $J_{2}$ to obtain the graph $3 K_{3}$. By Lemma 2.1.1 (v) and since $J_{1} \in\left\langle\mathcal{S} \mathbb{Z}_{5}\right\rangle$ is a spanning subgraph of $3 K_{3}$, we have $3 K_{3} \in\left\langle\mathcal{S} \mathbb{Z}_{5}\right\rangle$, which implies that the boundary $b$ at each vertex can be modified in $J_{2}$. If $b\left(x_{0}\right)=2$ or 3 , we lift the edges pair $\left\{x_{2} x_{0}, x_{0} x_{3}\right\}$ twice 
to obtain the graph $G_{1}$ and then orient the parallel edges from $x_{0}$ to $x_{1}$ or from $x_{1}$ to $x_{0}$ in $G_{1}$, respectively. By Lemma 2.1.1(iii), we could modify the boundary $b\left(x_{1}\right)$ by two pairs of parallel edges $x_{1} x_{2}, x_{1} x_{3}$ and then modify the boundaries $b\left(x_{2}\right)$ and $b\left(x_{3}\right)$ by the four parallel edges between $x_{2}$ and $x_{3}$. Thus the obtained orientation agrees with the boundary $b$. So we have $b\left(x_{i}\right) \in\{1,4\}$ for each $i$, and by symmetry, we may assume that $b\left(x_{0}\right)=b\left(x_{2}\right)=1$ and $b\left(x_{1}\right)=b\left(x_{3}\right)=4$. To agree with the boundary $b$ in this case, we orient two pairs of parallel edges $x_{1} x_{0}, x_{3} x_{0}$ toward $x_{0}$, two pairs of parallel edges $x_{1} x_{2}, x_{3} x_{2}$ toward $x_{2}$, two parallel edges $x_{0} x_{2}$ with opposite directions and two parallel edges $x_{1} x_{3}$ with opposite directions. Therefore, all possible boundaries $b$ are examined, and so $J_{2}$ is strongly $\mathbb{Z}_{5}$-connected by definition.

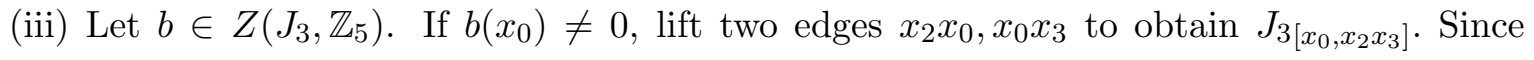
$b\left(x_{0}\right) \neq 0$ and $\left|\left[x_{0},\left\{x_{1}, x_{3}\right\}\right]_{J_{3\left[x_{0}, x_{2} x_{3}\right]}}\right|=3$, we can modify the boundary $b\left(x_{0}\right)$ with the three

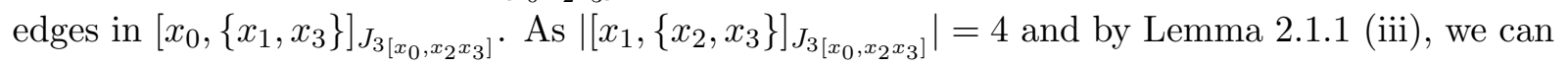
modify the boundary $b\left(x_{1}\right)$. Furthermore, as $\left|\left[x_{2}, x_{3}\right]_{J_{3\left[x_{0}, x_{2} x_{3}\right]}}\right|=4$ and by Lemma 2.1.1 (iii), we can modify the boundaries $b\left(x_{2}\right)$ and $b\left(x_{3}\right)$. Thus we assume $b\left(x_{0}\right)=0$. We lift the two edges $x_{2} x_{1}, x_{1} x_{3}$ to obtain $J_{3\left[x_{1}, x_{2} x_{3}\right]}$. Orient the five edges incident with $x_{0}$ out from $x_{0}$ in $J_{3\left[x_{1}, x_{2} x_{3}\right]}$. If $b\left(x_{1}\right)=0,1,3$ in $J_{3\left[x_{1}, x_{2} x_{3}\right]}$ we orient two edges from $x_{1}$ toward $x_{2}, x_{3}$, two edges from $x_{2}, x_{3}$ toward $x_{1}$, one edge from $x_{1}$ to $x_{2}$ and one edge from $x_{3}$ to $x_{1}$, respectively. If $b\left(x_{1}\right)=4,2$, reverse the above obtained orientation in $J_{3\left[x_{1}, x_{2} x_{3}\right]}$ corresponding to $b\left(x_{0}\right)=1,3$, respectively. Then modify the boundaries $b\left(x_{2}\right)$ and $b\left(x_{3}\right)$, by Lemma 2.1 .1 (iii) and $\left|\left[x_{2}, x_{3}\right]_{J_{3\left[x_{1}, x_{2} x_{3}\right]}}\right|=4$. Thus $J_{3}$ is strongly $\mathbb{Z}_{5}$-connected.

(iv) Since $J_{4}$ contains $J_{1}$ as a subgraph, $J_{4} / J_{1}=4 K_{2}$ and $J_{1} \in\left\langle\mathcal{S} \mathbb{Z}_{5}\right\rangle$, we conclude that $J_{4}$ is strongly $\mathbb{Z}_{5}$-connected by Lemma 2.1 .1 (iii)(v).

\subsection{Proofs of Main Results}

We shall present the proof of Theorem 1.3.2 first, and it would be used to prove Theorem 1.3.1.

\subsubsection{Proof of Theorem 1.3.2}

Define $\mathcal{F}_{n}=\left\{\left(d_{1}, \cdots, d_{n}\right): \sum_{i=1}^{n} d_{i}=8 n-8\right.$ and $\left.\min _{i \in[n]}\left\{d_{i}\right\} \geq 4\right\}$.

Lemma 2.2.1. Let $d=\left(d_{1}, d_{2}, \cdots, d_{n}\right) \in \mathcal{F}_{n}$ be a nonincreasing sequence. Then $d$ is multigraphic. Moreover, each of the following holds.

(i) If $n \geq 4$ and $\left(d_{n-1}, d_{n}\right) \in\{(5,5),(6,5)\}$, then there exist $\left(d_{1}^{\prime}, \cdots, d_{n-2}^{\prime}\right) \in \mathcal{F}_{n-2}$ and nonnegative integer $c_{j}$ such that for each $1 \leq j \leq n-2, d_{j}=d_{j}^{\prime}+c_{j}$ and

$$
\sum_{j=1}^{n-2} c_{j}= \begin{cases}6, & \text { if }\left(d_{n-1}, d_{n}\right)=(5,5) \\ 5, & \text { if }\left(d_{n-1}, d_{n}\right)=(6,5)\end{cases}
$$


(ii) If $n \geq 5$ and $\left(d_{n-2}, d_{n-1}, d_{n}\right) \in\{(7,7,5),(6,6,6),(7,6,6),(7,7,6)\}$, then there exist $\left(d_{1}^{\prime}, \cdots, d_{n-3}^{\prime}\right) \in \mathcal{F}_{n-3}$ and nonnegative integer $c_{j}$ such that for each $1 \leq j \leq n-3, d_{j}=d_{j}^{\prime}+c_{j}$ and

$$
\sum_{j=1}^{n-3} c_{j}= \begin{cases}5, & \text { if }\left(d_{n-2}, d_{n-1}, d_{n}\right)=(7,7,5) \\ 6, & \text { if }\left(d_{n-2}, d_{n-1}, d_{n}\right)=(6,6,6) \\ 5, & \text { if }\left(d_{n-2}, d_{n-1}, d_{n}\right)=(7,6,6) \\ 4, & \text { if }\left(d_{n-2}, d_{n-1}, d_{n}\right)=(7,7,6)\end{cases}
$$

Proof. Since $d_{n} \geq 4$, we have $\sum_{i=2}^{n} d_{i} \geq 4 n-4$. Then $d_{1} \leq \sum_{i=1}^{n} d_{i}-(4 n-4)=4 n-4 \leq \sum_{i=2}^{n} d_{i}$. By Theorem 3.3.3, $d$ is multigraphic.

(i) Denote $k=16-d_{n-1}-d_{n}$. If $n \geq 4$, then by $\sum_{i=1}^{n} d_{i}=8 n-8$, we have

$$
\sum_{i=1}^{n} d_{i}=8 n-8 \geq 4(n-2)+16=4(n-2)+\left(d_{n}+d_{n-1}\right)+k .
$$

Thus there exists a minimal integer $i_{0} \in[n-2]$ such that $\sum_{j=1}^{i_{0}} d_{j} \geq 4 i_{0}+k$. Let $c_{j}=d_{j}-4$ for $1 \leq j \leq i_{0}-1, c_{i_{0}}=k-\sum_{j=1}^{i_{0}-1} d_{j}$ and $c_{j}=0$ if $i_{0}+1 \leq j \leq n-2$. Let $d_{j}^{\prime}=d_{j}-c_{j}$ for each $1 \leq j \leq n-2$. Then the degree sequence $\left(d_{1}^{\prime}, \cdots, d_{n-2}^{\prime}\right) \in \mathcal{F}_{n-2}$ since

$$
\sum_{j=1}^{n-2} d_{j}^{\prime}=\sum_{j=1}^{n-2} d_{j}-\sum_{j=1}^{n-2} c_{j}=\sum_{j=1}^{n-2} d_{j}-k=\sum_{j=1}^{n} d_{j}-16=8(n-2),
$$

and $d_{j}^{\prime} \geq 4$ for each $1 \leq j \leq n-2$. Moreover, Eq. (2.1) is satisfied as well.

(ii) The proof is similar to (i). Denote $t=24-d_{n-2}-d_{n-1}-d_{n}$. If $n \geq 5$, then by $\sum_{i=1}^{n} d_{i}=8 n-8$, we obtain

$$
\sum_{i=1}^{n} d_{i}=8 n-8 \geq 4(n-3)+24=4(n-3)+\left(d_{n}+d_{n-1}+d_{n-2}\right)+t .
$$

Thus there exists a minimal integer $i_{0} \in[n-3]$ such that $\sum_{j=1}^{i_{0}} d_{j} \geq 4 i_{0}+t$. Let $c_{j}=d_{j}-4$ for $1 \leq j \leq i_{0}-1, c_{i_{0}}=t-\sum_{j=1}^{i_{0}-1} d_{j}$ and $c_{j}=0$ if $i_{0}+1 \leq j \leq n-3$. Let $d_{j}^{\prime}=d_{j}-c_{j}$ for $1 \leq j \leq n-3$. Then we obtain that $\left(d_{1}^{\prime}, \cdots, d_{n-3}^{\prime}\right) \in \mathcal{F}_{n-3}$ as

$$
\sum_{j=1}^{n-3} d_{j}^{\prime}=\sum_{j=1}^{n-3} d_{j}-\sum_{j=1}^{n-3} c_{j}=\sum_{j=1}^{n-3} d_{j}-t=\sum_{j=1}^{n} d_{j}-24=8(n-3),
$$

and $d_{j}^{\prime} \geq 4$ for each $1 \leq j \leq n-3$. Furthermore, Eq. (2.2) holds as well.

To prove Theorem 1.3.2, we verify the following key Lemma first. 
Lemma 2.2.2. For any nonincreasing multigraphic sequence $d=\left(d_{1}, d_{2}, \cdots, d_{n}\right)$ with $\sum_{i=1}^{n} d_{i}=$ $8 n-8$ and $d_{n} \geq 4$, $d$ has a strongly $\mathbb{Z}_{5}$-connected realization.

Proof. We apply induction on $n$. If $2 \leq n \leq 3$, then all the degree sequences satisfying the assumption $\sum_{i=1}^{n} d_{i}=8 n-8$ and $d_{n} \geq 4$ are depicted below in Figure 2.2.

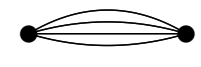

$(4,4)$

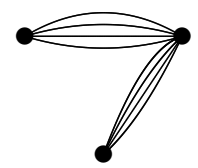

$(8,4,4)$

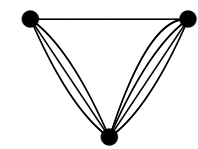

$(7,5,4)$

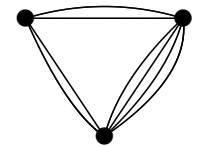

$(6,6,4)$

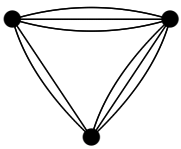

$(6,5,5)$

Figure 2.2: The graphs in Lemma 2.2.2.

It follows from Lemma 2.1.1 (iii)(v) and Lemma 2.1.7 that each graph above is strongly $\mathbb{Z}_{5}$-connected, and so Lemma 2.2.2 holds if $2 \leq n \leq 3$. Thus we assume $n \geq 4$ and Lemma 2.2.2 holds for integers smaller than $n$. Notice that $4 \leq d_{n} \leq 7$, since $\sum_{i=1}^{n} d_{i}=8 n-8$.

Case 1: $d_{n}=4$.

Since $\sum_{i=1}^{n-1} d_{i}=8 n-12 \geq 4(n-1)+4$, similar to the proof of Lemma 2.2.1, there exists a sequence $d^{\prime}=\left(d_{1}^{\prime}, \cdots, d_{n-1}^{\prime}\right)$ and nonnegative integer $c_{i}$ for each $i \in[n-1]$ such that $\sum_{i=1}^{n-1} c_{i}=4, d_{i}=d_{i}^{\prime}+c_{i}$ and $d_{i}^{\prime} \geq 4$. Then $\sum_{i=1}^{n-1} d_{i}^{\prime}=8(n-1)-d_{n}-\sum_{i=1}^{n} c_{i}=8(n-2)$. By Lemma 2.2.1, $d^{\prime}$ is multigraphic and $d^{\prime}$ has a strongly $\mathbb{Z}_{5}$-connected realization $G^{\prime}$ by induction on $n$. Let $G$ be the graph obtained from $G^{\prime}$ by adding one new vertex $v_{n}$ and $c_{i}$ edges joining the vertex $v_{n}$ with $d_{i}^{\prime}$-vertex for each $1 \leq i \leq n-1$. As $G / G^{\prime}=4 K_{2} \in\left\langle\mathcal{S} \mathbb{Z}_{5}\right\rangle$ and $G^{\prime} \in\left\langle\mathcal{S} \mathbb{Z}_{5}\right\rangle$, $G$ is a strongly $\mathbb{Z}_{5}$-connected realization of $d$ by Lemma 2.1 .1 (iii)(v).

Case $2: d_{n}=5$ or $d_{n}=6$.

In this case, we shall divide our discussion according to $\left(d_{n-1}, d_{n}\right)$ or $\left(d_{n-2}, d_{n-1}, d_{n}\right)$.

If $\left(d_{n-1}, d_{n}\right) \in\{(5,5),(6,5)\}$, by Lemma 2.2.1(i), there exists $d^{\prime}=\left(d_{1}^{\prime}, d_{2}^{\prime}, \cdots, d_{n-2}^{\prime}\right) \in$ $\mathcal{F}_{n-2}$ such that $d_{i}=d_{i}^{\prime}+c_{i}$ where $\sum_{i=1}^{n-2} c_{i}=6$ if $\left(d_{n-1}, d_{n}\right)=(5,5)$ and $\sum_{i=1}^{n-2} c_{i}=5$ if $\left(d_{n-1}, d_{n}\right)=(6,5)$. By Lemma 2.2.1, $d^{\prime}$ is multigraphic. By induction on $n, d^{\prime}$ has a strongly $Z_{5}$-connected realization $G^{\prime}$. Construct the graph $G$ from $G^{\prime}$ by adding two new vertices $v_{n-1}, v_{n}$ with $\left\lceil\frac{16-\sum_{i=1}^{n-2} c_{i}}{5}\right\rceil$ parallel edges $v_{n} v_{n-1}$ and for each $i \in[n-2]$, joining $c_{i}$ edges from the $d_{i^{-}}^{\prime}$ vertex to $\left\{v_{n-1}, v_{n}\right\}$ to obtain a new graph $G$ as a $d$-realization. Since $G / G^{\prime}=J_{1}$ (see Figure 2.1), $G^{\prime} \in\left\langle\mathcal{S} \mathbb{Z}_{5}\right\rangle$ and $J_{1} \in\left\langle\mathcal{S} \mathbb{Z}_{5}\right\rangle$ by Lemma 2.1 .7 , we conclude that $G$ is a strongly $\mathbb{Z}_{5}$-connected realization of $d$ by Lemma $2.1 .1(\mathrm{v})$.

If $n \geq 5$ and $\left(d_{n-2}, d_{n-1}, d_{n}\right) \in\{(7,7,5),(6,6,6),(7,6,6),(7,7,6)\}$, by Lemma 2.2 .1 (ii), there exists $d^{\prime}=\left(d_{1}^{\prime}, d_{2}^{\prime}, \cdots, d_{n-3}^{\prime}\right) \in \mathcal{F}_{n-3}$ satisfying $d_{i}=d_{i}^{\prime}+c_{i}$ and Eq. (2.2). Since $\sum_{i=1}^{n-3} d_{i}^{\prime}=$ 
$8(n-4)$ and $\min _{i \in[n-3]} d_{i}^{\prime} \geq 4$ and by Lemma 2.2.1, $d^{\prime}$ is multigraphic. Then $d^{\prime}$ has a strongly $\mathbb{Z}_{5}$-connected realization $G^{\prime}$, by induction on $n$.

If $\left(d_{n-2}, d_{n-1}, d_{n}\right)=(7,7,5)$, let $A=\left\{v \in V\left(G^{\prime}\right): v\right.$ is a $d_{i}^{\prime}$-vertex with $c_{i}>0$ and $i \in$ $[n-3]\}$. We construct a graph $G$ from $G^{\prime}$ by adding three new vertices $v_{n-2}, v_{n-1}, v_{n}$ and 12 edges such that $\left|\left[v_{n}, v_{n-1}\right]_{G}\right|=3,\left|\left[v_{n-2}, v_{n-1}\right]_{G}\right|=4,\left|\left[v_{n}, A\right]_{G}\right|=2,\left|\left[v_{n-2}, A\right]_{G}\right|=3$ to obtain a new graph $G$ so that $G$ is a $d$-realization. By Lemma 2.1.7 and Lemma 2.1.1 (iii)(v), as $G^{\prime} \in\left\langle\mathcal{S} \mathbb{Z}_{5}\right\rangle$ and $G / G^{\prime} /\left[v_{n-1}, v_{n-2}\right]_{G}=J_{1} \in\left\langle\mathcal{S} \mathbb{Z}_{5}\right\rangle$, we have $G \in\left\langle\mathcal{S} \mathbb{Z}_{5}\right\rangle$, which provides a strongly $\mathbb{Z}_{5}$-connected realization of $d$.

If $\left(d_{n-2}, d_{n-1}, d_{n}\right)=(6,6,6)$, let $B=\left\{v \in V\left(G^{\prime}\right): v\right.$ is a $d_{i}^{\prime}$-vertex with $c_{i}>0$ and $i \in$ $[n-3]\}$. We construct a graph $G$ from $G^{\prime}$ by adding three new vertices $v_{n-2}, v_{n-1}, v_{n}$ and 12 edges such that $\left|\left[v_{n}, v_{n-1}\right]_{G}\right|=2,\left|\left[v_{n-2}, v_{n-1}\right]_{G}\right|=2,\left|\left[v_{n-2}, v_{n}\right]_{G}\right|=2,\left|\left[v_{n}, B\right]_{G}\right|=2$, $\left|\left[v_{n-2}, B\right]_{G}\right|=2$ and $\left|\left[v_{n-1}, B\right]_{G}\right|=2$ to obtain a new graph $G$ so that $G$ is a $d$-realization. By Lemma 2.1.7 and Lemma 2.1.1 (v), as $G^{\prime} \in\left\langle\mathcal{S} \mathbb{Z}_{5}\right\rangle$ and $G / G^{\prime}=J_{2} \in\left\langle\mathcal{S} \mathbb{Z}_{5}\right\rangle$, we have $G \in\left\langle\mathcal{S} \mathbb{Z}_{5}\right\rangle$, which provides a strongly $\mathbb{Z}_{5}$-connected realization of $d$.

If $\left(d_{n-2}, d_{n-1}, d_{n}\right)=(7,6,6)$, let $C=\left\{v \in V\left(G^{\prime}\right): v\right.$ is a $d_{i}^{\prime}$-vertex with $c_{i}>0$ and $i \in$ $[n-3]\}$. We construct a graph $G$ from $G^{\prime}$ by adding three new vertices $v_{n-2}, v_{n-1}, v_{n}$ and 12 edges such that $\left|\left[v_{n}, v_{n-1}\right]_{G}\right|=3,\left|\left[v_{n-2}, v_{n-1}\right]_{G}\right|=2,\left|\left[v_{n-2}, v_{n}\right]_{G}\right|=2,\left|\left[v_{n}, C\right]_{G}\right|=2$, $\left|\left[v_{n-1}, C\right]_{G}\right|=1$ and $\left|\left[v_{n-2}, C\right]_{G}\right|=2$ to obtain a new graph $G$ so that $G$ is a $d$-realization. By Lemma 2.1.7 and Lemma 2.1.1 (v), we have $G \in\left\langle\mathcal{S} \mathbb{Z}_{5}\right\rangle$ as $G^{\prime} \in\left\langle\mathcal{S} \mathbb{Z}_{5}\right\rangle$ and $G / G^{\prime}=J_{3} \in\left\langle\mathcal{S} \mathbb{Z}_{5}\right\rangle$.

If $\left(d_{n-2}, d_{n-1}, d_{n}\right)=(7,7,6)$, let $D=\left\{v \in V\left(G^{\prime}\right): v\right.$ is a $d_{i}^{\prime}$-vertex with $c_{i}>0$ and $i \in$ $[n-3]\}$. We construct a graph $G$ from $G^{\prime}$ by adding three new vertices $v_{n-2}, v_{n-1}, v_{n}$ and 12 edges such that $\left|\left[v_{n}, v_{n-1}\right]_{G}\right|=3,\left|\left[v_{n-2}, v_{n-1}\right]_{G}\right|=3,\left|\left[v_{n-2}, v_{n}\right]_{G}\right|=2,\left|\left[v_{n}, D\right]_{G}\right|=1$, $\left|\left[v_{n-2}, D\right]_{G}\right|=2$ and $\left|\left[v_{n-1}, D\right]_{G}\right|=1$ to obtain a new graph $G$ so that $G$ is a $d$-realization. Since $G^{\prime} \in\left\langle\mathcal{S} \mathbb{Z}_{5}\right\rangle$ and $G / G^{\prime}=J_{4} \in\left\langle\mathcal{S} \mathbb{Z}_{5}\right\rangle$, we have $G \in\left\langle\mathcal{S} \mathbb{Z}_{5}\right\rangle$ as $d$-realization by Lemma 2.1 .7 and Lemma 2.1.1(v).

Similarly, if $\left(d_{n-2}, d_{n-1}, d_{n}\right) \in\{(6,6,6),(7,6,6),(7,7,6)\}$, we accordingly construct a graph $G$ such that $G / G^{\prime} \in\left\{J_{2}, J_{3}, J_{4}\right\}$ respectively, and $x_{0} \in V(J)$ with $J \in\left\{J_{2}, J_{3}, J_{4}\right\}$ (see Figure 1 ) is the vertex onto which $G^{\prime}$ is contracted in $G / G^{\prime}$. Thus $d$ has a realization $G$. By Lemma 2.1.1 (v) and Lemma 2.1.7, $G$ is a strongly $\mathbb{Z}_{5}$-connected realization of $d$.

The remaining case is $n=4$ and $\sum_{i=1}^{4} d_{i}=24$, and then $\left(d_{1}, d_{2}, d_{3}, d_{4}\right)=(6,6,6,6)$. By Lemma 2.1.7, the graph $J_{2}$ (see Figure 1) is the desired graph.

Case $3: d_{n}=7$.

If $d_{n}=7$, by $\sum_{i=1}^{n} d_{i}=8 n-8$, then $d_{n}=d_{n-1}=\cdots=d_{n-6}=7$, which implies that $n \geq 7$. Thus

$$
\sum_{i=1}^{n-4} d_{i}=8 n-8-28 \geq 4(n-4)+4 .
$$

By a similar argument as in Lemma 2.2.1, there exists a degree sequence $d^{\prime}=\left(d_{1}^{\prime}, \cdots, d_{n-4}^{\prime}\right)$ and 
nonnegative integer $c_{i}$ such that $d_{i}=d_{i}^{\prime}+c_{i}$ and $d_{i}^{\prime} \geq 4$ for $1 \leq i \leq n-4$, where $\sum_{i=1}^{n-4} c_{i}=4$. Thus

$$
\sum_{i=1}^{n-4} d_{i}^{\prime}=\sum_{i=1}^{n} d_{i}-\sum_{i=n-3}^{n} d_{i}-\sum_{i=1}^{n-4} c_{i}=8(n-1)-28-4=8(n-5) .
$$

By Lemma 2.2.1, $d^{\prime}$ is multigraphic. By induction on $n, d^{\prime}$ has a strongly $\mathbb{Z}_{5}$-connected realization $G^{\prime}$. We construct the graph $G$ from $G^{\prime}$ and $3 C_{4}$ by adding $c_{i}$ edges between $d_{i}^{\prime}$-vertex and vertices of $3 C_{4}$ such that $d_{G}(x)=7$ for any $x \in V\left(3 C_{4}\right)$ so that $G$ is a $d$-realization. By Lemma 2.1.1 (ii) and Theorem 2.1.2 (i), $3 C_{4} \in\left\langle\mathcal{S} \mathbb{Z}_{5}\right\rangle$. By Lemma 2.1.1 (iii) (v) and $\left(G / G^{\prime}\right) / 3 C_{4}=4 K_{2} \in\left\langle\mathcal{S} \mathbb{Z}_{5}\right\rangle$, $G$ is a strongly $\mathbb{Z}_{5}$-connected $d$-realization. This completes the proof.

Now we are ready to prove Theorem 1.3.2.

Theorem 1.3.2 For any nonincreasing multigraphic sequence $d=\left(d_{1}, d_{2}, \cdots, d_{n}\right)$, $d$ has a strongly $\mathbb{Z}_{5}$-connected realization if and only if $\sum_{i=1}^{n} d_{i} \geq 8 n-8$ and $d_{n} \geq 4$.

Proof. To prove the necessarity, by Theorem 2.1.2 (ii) and Lemma 2.1.1 (iii), if $G \in\left\langle\mathcal{S} \mathbb{Z}_{5}\right\rangle$ with degree sequence $\left(d_{1}, d_{2}, \cdots, d_{n}\right)$, then $\sum_{i=1}^{n} d_{i} \geq 8 n-8$ and $d_{n} \geq 4$.

For sufficiency, suppose the contrary that the nonincreasing multigraphic sequence $\left(d_{1}, d_{2}, \cdots, d_{n}\right)$ is a counterexample with $\sum_{i=1}^{n} d_{i}$ minimized. By Lemma 2.2.2, $\sum_{i=1}^{n} d_{i}>8 n-8$ and $d_{n} \geq 4$. If $d_{2}=4$, then by Theorem 3.3.3, we have $\sum_{i=1}^{n} d_{i} \leq 2 \sum_{i=2}^{n} d_{i}=8 n-8$, a contradiction. Thus we assume $d_{2} \geq 5$ and let $\left(d_{1}^{\prime}, d_{2}^{\prime}, d_{3}^{\prime} \cdots, d_{n}^{\prime}\right)=\left(d_{1}-1, d_{2}-1, d_{3}, \cdots, d_{n}\right)$. By Theorem 2.1.4, $\left(d_{1}^{\prime}, \cdots, d_{n}^{\prime}\right)$ is multigraphic, and so by the minimality of $d,\left(d_{1}^{\prime}, \cdots, d_{n}^{\prime}\right)$ has a strongly $\mathbb{Z}_{5}$-connected realization $G^{\prime}$. Then we obtain the graph $G$ as a $d$-realization from $G^{\prime}$ by adding one edge between the $d_{1}^{\prime}$-vertex and the $d_{2}^{\prime}$-vertex. Since $G^{\prime} \in\left\langle\mathcal{S} \mathbb{Z}_{5}\right\rangle$, it follows from Lemma 2.1.1(v) that $G \in\left\langle\mathcal{S} \mathbb{Z}_{5}\right\rangle$, a contradiction.

\subsubsection{Proof of Theorem 1.3.1}

Theorem 1.3.1 For any nonincreasing multigraphic sequence $d=\left(d_{1}, d_{2}, \cdots, d_{n}\right)$, $d$ has a modulo 5-orientation realization if and only if $d_{i} \notin\{1,3\}$ for every $1 \leq i \leq n$.

Proof. To prove the necessarity, let $\left(d_{1}, \cdots, d_{n}\right)$ be any multigraphic sequence, by the definition of modulo 5-orientation, we achieve $d_{i} \notin\{1,3\}$ for every $1 \leq i \leq n$.

For sufficiency, suppose the contrary that the nonincreasing multigraphic sequence $d=$ $\left(d_{1}, \cdots, d_{n}\right)$ is a counterexample with $m=\sum_{i=1}^{n} d_{i}$ minimized. By Theorem $3.3 .3, d_{1} \leq \sum_{i=2}^{n} d_{i}$.

Claim A: $d_{1} \leq \sum_{i=2}^{n} d_{i}-4$.

By contradiction, we assume $d_{1} \in\left\{\sum_{i=2}^{n} d_{i}-2, \sum_{i=2}^{n} d_{i}\right\}$. 
If $d_{1}=\sum_{i=2}^{n} d_{i}$, then $d$ has a unique realization $G$ by setting $v_{1}$ as the center vertex adjacent to the vertices $v_{2}, \cdots, v_{n}$ with $d_{2}, \cdots, d_{n}$ multiple edges, respectively. Now we are to prove that $G$ has a modulo 5-orientation $D$. For each $2 \leq i \leq n-1$, if $d_{i}$ is even, then we orient one half of the edges from $v_{i}$ toward $v_{1}$ and orient rest edges from $v_{1}$ to $v_{i}$. If $d_{i}$ is odd, we assign $\frac{d_{i}+5}{2}$ edges with the orientation from $v_{i}$ into vertex $v_{1}$ and $\frac{d_{i}-5}{2}$ edges with opposite direction. Thus $G$ is a modulo 5-orientation realization of $d$, a contradiction.

Assume that $d_{1}=\sum_{i=2}^{n} d_{i}-2$. From the above oriented graph $G$ with degree sequence $\left(\sum_{i=2}^{n} d_{i}, d_{2}, \cdots, d_{n}\right)$, we pick up one directed edge oriented into the vertex $v_{1}$, denoted by $e_{1}$, and another edge oriented out from $v_{1}$, denoted by $e_{2}$, where $e_{1} \cap e_{2}=\left\{v_{1}\right\}$. Let $G^{\prime}$ be the graph obtained from $G$ by lifting two edges $e_{1}, e_{2}$ to become a new edge. It is obvious to see that $G^{\prime}$ preserves the modulo 5-orientation and moreover $G^{\prime}$ has degree sequence $d=\left(\sum_{i=2}^{n} d_{i}-2, d_{2}, \cdots, d_{n}\right)$. This contradicts to the assumption that $d$ is a counterexample.

Claim B: $d_{n} \notin\{2,4\}$ and $n \geq 4$.

By contradiction, assume that $d_{n}=2 t$ for some $t \in\{1,2\}$. Let $d^{\prime}=\left(d_{1}^{\prime}, d_{2}^{\prime}, \cdots, d_{n-1}^{\prime}\right)=$ $\left(d_{1}, d_{2}, \cdots, d_{n-1}\right)$. Since $d_{1} \leq \sum_{i=2}^{n} d_{i}-4$ by Claim A, we have $d_{1}^{\prime} \leq \sum_{i=2}^{n-1} d_{i}^{\prime}$. By Theorem 3.3.3, $d^{\prime}$ is multigraphic. Since $\sum_{i=1}^{n-1} d_{i}^{\prime}<m$ and by the minimality of $m, d^{\prime}$ has a modulo 5-orientation realization $G^{\prime}$. We pick up $t$ directed edges $e_{1}, \cdots, e_{t}$ in the modulo 5-orientation of $G^{\prime}$. Let $G$ be the graph obtained from $G^{\prime}$ by inserting the edges $e_{1}, \cdots, e_{t}$ to a new vertex $v_{n}$. This would extend the modulo 5-orientation of $G^{\prime}$ to the graph $G$. However, it is clear that $G$ is a $d$-realization, a contradiction.

The case of $n=2$ is obvious. Let $n=3$. Since $d_{3} \geq 5$, we have $d_{1}+d_{2}+d_{3} \geq 15$, and so $d_{1}+d_{2}+d_{3} \geq 8(n-1)=16$ by parity. By Theorem $1.3 .2, d$ has a strongly $Z_{5}$-connected realization, and therefore a modulo 5-orientation realization, a contradiction.

Claim C: $d_{1} \leq \sum_{i=2}^{n} d_{i}-6$ and $d_{n} \neq 6$.

Suppose to the contrary that $d_{1}=\sum_{i=2}^{n} d_{i}-4$ (by Claim A). Similar to the proof of Claim A, let $G$ be a $\left(\sum_{i=2}^{n} d_{i}, d_{2}, \cdots, d_{n}\right)$-realization with center vertex $v_{1}$ adjacent to the vertices $v_{2}, \cdots, v_{n}$ with $d_{2}, \cdots, d_{n}$ multiple edges, respectively. Since $d_{n-1} \geq d_{n} \geq 5$ by Claim B, we lift the edges pair $\left\{v_{1} v_{n-1}, v_{1} v_{n}\right\}$ twice to obtain a graph $G^{\prime}$. Then $G^{\prime}\left[\left\{v_{1}, v_{n-1}, v_{n}\right\}\right]$ contains the graph $J_{1}$ (see Figure 1), and therefore has a modulo 5-orientation by Lemma 2.1.7. Since $\left|\left[v_{1}, v_{i}\right]_{G^{\prime}}\right| \geq 5$ for each $2 \leq i \leq n-2$, we can extend the modulo 5-orientation of $G^{\prime}\left[\left\{v_{1}, v_{n-1}, v_{n}\right\}\right]$ to the entire graph $G^{\prime}$ by Lemma 2.1 .1 (iii). This shows that $G^{\prime}$ is a modulo 5-orientation $d$ realization, a contradiction.

We observe that $d_{n} \neq 6$ follows from a similar argument as in Claim B, since $\left(d_{1}, d_{2}, \cdots, d_{n-1}\right)$ is multigraphic provided that $d_{n}=6$ and $d_{1} \leq \sum_{i=2}^{n} d_{i}-6$. That is, we can insert three edges in $G^{\prime}$ to a new vertex $v_{n}$ to form the desired graph $G$. 
Now, as $d_{n} \geq 5$ and by Theorem 1.3 .2 , we have

$$
\sum_{i=1}^{n} d_{i} \leq 8 n-10
$$

Claim D: $d_{n} \neq 5$.

If $n=4$ and $d_{4}=5$, then by $\sum_{i=1}^{4} d_{i} \leq 22, d=\left(d_{1}, d_{2}, d_{3}, d_{4}\right) \in\{(5,5,5,5),(7,5,5,5),(6,6,5,5)\}$. If $\left(d_{1}, d_{2}, d_{3}, d_{4}\right) \in\{(5,5,5,5),(6,6,5,5)\}$, we obtain the desired graph $G$ from $J_{1}$ in Figure 1 by replacing the vertex $x_{3}$ with 2 or 3 parallel edges, separately. If $\left(d_{1}, d_{2}, d_{3}, d_{4}\right)=(7,5,5,5)$, then we have the graph $G$ from $J_{1}$ by inserting the parallel edges $x_{1} x_{2}$ to a new vertex $x_{4}$ and adding one new edge $x_{3} x_{4}$. In any case, it is easy to check that $G$ is a modulo 5-orientation $d$-realization, a contradiction.

If $n \geq 5$ and $d_{n}=d_{n-1}=5$, then let $d^{\prime}=\left(d_{1}^{\prime}, d_{2}^{\prime}, \cdots, d_{n-2}^{\prime}\right)=\left(d_{1}, d_{2}, \cdots, d_{n-2}\right)$. Since

$$
d_{1}+5(n-1) \leq d_{1}+\sum_{i=2}^{n} d_{i} \leq 8 n-10,
$$

we obtain $d_{1} \leq 3 n-5$. Since $n \geq 5, d_{1}^{\prime} \leq 3 n-5 \leq 5(n-3) \leq \sum_{i=2}^{n-2} d_{i}^{\prime}$. By Theorem 3.3.3, $d^{\prime}$ is multigraphic. By induction, $d^{\prime}$ has a modulo 5-orientation realization $G^{\prime}$. Pick up a directed edge $u v$ in the graph $G^{\prime}$. Construct the graph $G$ from $G^{\prime}$ by adding distinct vertices $v_{n-1}, v_{n}$, deleting oriented edge $u v$ and adding oriented edges $u v_{n-1}, v_{n} v$ and 4 parallel edges $v_{n} v_{n-1}$. Thus $G$ is the desired graph by Lemma 2.1.1 (iii), a contradiction.

Otherwise, since $d_{n}=5$ and $\sum_{i=1}^{n} d_{i}$ is even, there exists an odd $d_{i} \geq 7$ for some $1 \leq$ $i \leq n-1$. Let $d^{\prime}=\left(d_{1}^{\prime}, \cdots, d_{i}^{\prime}, \cdots, d_{n-1}^{\prime}\right)=\left(d_{1}, \cdots, d_{i}-5, \cdots, d_{n-1}\right)$. Since $n \geq 5$, we have $d_{1}^{\prime}=d_{1} \leq 3 n-5 \leq 5(n-3)+2 \leq \sum_{i=2}^{n-1} d_{i}^{\prime}$. By Theorem 3.3.3 and induction, let $G^{\prime}$ be a modulo 5-orientation realization of $d^{\prime}$. Construct the graph $G$ from $G^{\prime}$ by adding a new vertex $v_{n}$ such that $v_{n}$ is adjacent to the $d_{i}^{\prime}$-vertex with 5 parallel edges. By Lemma 2.1.1 (iii), $G$ is a modulo 5-orientation $d$-realization, a contradiction.

Claim E: $d_{n} \neq 7$.

If $d_{n}=7$, then $d_{n}=d_{n-1}=\cdots=d_{n-6}=7$ by Eq. (2.3), which implies that $n \geq 7$. Let $d^{\prime}=\left(d_{1}^{\prime}, \cdots, d_{n-4}^{\prime}\right)=\left(d_{1}, \cdots, d_{n-4}\right)$. Since $d_{1}+7(n-1) \leq \sum_{i=1}^{n} d_{i} \leq 8 n-10$, we obtain $d_{1}^{\prime} \leq n-3 \leq 7(n-5) \leq \sum_{i=2}^{n-4} d_{i}^{\prime}$. By Theorem 3.3.3 and induction, $d^{\prime}$ has a modulo 5-orientation realization $G^{\prime}$. Let $u_{1} v_{1}, u_{2} v_{2}$ be two directed distinct edges in $G^{\prime}$. We construct the graph $G$ from $G^{\prime}$ and $3 C_{4}$ with vertices $v_{j}, n-3 \leq j \leq n$, by deleting $u_{1} v_{1}, u_{2} v_{2}$ and adding oriented edges $u_{1} v_{n-3}, v_{n-2} v_{1}, u_{2} v_{n-1}, v_{n} v_{2}$. By Lemma 2.1.1 (ii) and Theorem 2.1.1 (i), $3 C_{4}$ is strongly $\mathbb{Z}_{5}$-connected. Thus the modulo 5-orientation of $G^{\prime}$ is easily extended to the graph $G$ as a $d$-realization, a contradiction. 
Therefore, it follows from Claims A-E that $d_{n} \geq 8$, and so $\sum_{i=1}^{n} d_{i} \geq 8 n$, a contradiction to Eq. (2.3). The proof is completed.

\subsubsection{Proof of Theorem 1.3.3}

A graph is called cubic if it is 3-regular. For a cubic graph $G$, a $Y-\Delta$ operation on a vertex $v$ is to replace the vertex $v$ with a triangle, where each edge incident with $v$ in $G$ becomes an edge incident to a vertex of the triangle. It is clear that applying $Y-\Delta$ operation on a cubic graph still results a cubic graph, and it follows from Lemma 2.1.1(i)(ii) that any graph obtained from $K_{4}$ by $Y-\Delta$ operation is $\mathbb{Z}_{5}$-connected. We will use this observation (and in fact a stronger property) in the proof of Theorem 1.3.3. Before presenting the proof, we shall handle some special cases first. If a sequence $d$ consists of the terms $d_{1}, \ldots, d_{t}$ having multiplicities $m_{1}, \ldots, m_{t}$, we may write $d=\left(d_{1}^{m_{1}}, \ldots, d_{t}^{m_{t}}\right)$ for convenience.

Lemma 2.2.3. Each of the integral multigraphic sequences $\left(17,9^{3}\right),\left(14,9^{4}\right),\left(16,9^{4}\right),\left(16,9^{6}\right)$ has a 9-edge-connected strongly $\mathbb{Z}_{5}$-connected realization.

Proof. For $d=\left(17,9^{3}\right)$, we construct a graph $G$ as $d$-realization from $J_{1}$ in Figure 2.1 by adding a new vertex $x_{4}$ with 2 parallel edges $x_{1} x_{4}$ and 7 multiple edges $x_{2} x_{4}$, respectively, then adding 3,2 multiple edges $x_{3} x_{2}, x_{1} x_{2}$, respectively. It is routine to check that $G$ is 9-edge-connected, i.e. for any $S \subset V(G)$ with $|S|=1$ or 2 , we have $\left|[S, V(G) \backslash S]_{G}\right| \geq 9$. By Lemma 2.1 .7 and Lemma 2.1 .1 (iii)(v), $G$ is a strongly $\mathbb{Z}_{5}$-connected $d$-realization.

For $d=\left(16,9^{6}\right)$, we construct the graph $G_{0}$ from two disjoint copies of $3 K_{4}$ with labeled vertices $v^{\prime}, v^{\prime \prime}$ respectively, by identifying vertices $v^{\prime}, v^{\prime \prime}$ to a new vertex and lifting the two edges $e_{1}, e_{2}$, where $e_{1}, e_{2}$ are adjacent to $v^{\prime}, v^{\prime \prime}$ in each $3 K_{4}$. It is easy to check that $G_{0}$ is 9 -edgeconnected. Since $G_{0}$ contains $J_{2}$ (see Figure 1) as a subgraph and by Lemma 2.1.7 and Lemma $2.1 .1(\mathrm{v}), G_{0}$ is a strongly $\mathbb{Z}_{5}$-connected $d$-realization.

For $d=\left(16,9^{4}\right)$, we obtain the desired graph $G_{1}$ gained from $J_{1}$ in Figure 2.1 by adding two new vertices $x_{4}, x_{5}$ with edges $x_{1} x_{4}, x_{2} x_{4}$ and $3,3,3,7$ parallel edges $x_{3} x_{5}, x_{1} x_{5}, x_{2} x_{5}, x_{4} x_{5}$, respectively. For any $S \subset V\left(G_{1}\right)$, it is easy to check that $\left|\left[S, V\left(G_{1}\right) \backslash S\right]\right| \geq 9$. Thus $G_{1}$ is a 9-edge-connected strongly $\mathbb{Z}_{5}$-connected $d$-realization by Lemma 2.1.7 and Lemma 2.1.1 (iii)(v).

For $d=\left(14,9^{4}\right)$, we have the desired graph $G_{2}$ obtained from above $G_{1}$ by lifting the two edges $x_{3} x_{5}$ and $x_{4} x_{5}$. Let $S \subset V\left(G_{2}\right)$. It is routine to verify that $\left|\left[S, V\left(G_{2}\right) \backslash S\right]_{G_{2}}\right| \geq 9$ for any $S \subset V\left(G_{2}\right)$. Therefore $G_{2}$ is a 9 -edge-connected strongly $\mathbb{Z}_{5}$-connected $d$-realization by Lemma 2.1.7 and Lemma 2.1.1 (iii)(v).

Theorem 1.3.3 For any nonincreasing multigraphic sequence $d=\left(d_{1}, d_{2}, \cdots, d_{n}\right)$ with $\min _{i \in[n]} d_{i} \geq$ 9, d has a 9-edge-connected strongly $\mathbb{Z}_{5}$-connected realization. 
Proof. Let $d=\left(d_{1}, d_{2}, \cdots, d_{n}\right)$ be a nonincreasing multigraphic sequence with $d_{n} \geq 9$. By Theorem 3.3.3, we have $d_{1} \leq \sum_{i=2}^{n} d_{i}$. If $n=2$, then $d_{1}=d_{2}$ and it is obvious to verify this statement by Lemma 2.1.1(iii). We argue by induction on $m=\sum_{i=1}^{n} d_{i}$ and assume that $n \geq 3$ and that Theorem 1.3.3 holds for smaller value of $m$. We are to construct a 9-edge-connected strongly $\mathbb{Z}_{5}$-connected $d$-realization.

Case 1: $d_{1}=9$.

Since $d_{n} \geq 9$, we have $\left(d_{1}, d_{2}, \cdots, d_{n}\right)=(9,9, \cdots, 9)$. Since $\sum_{i=1}^{n} d_{i}$ is even and $n \geq 3$, this implies that $n$ is even and $n \geq 4$. We obtain a graph $G^{\prime}$ by applying $Y-\Delta$ operation on the complete graph $K_{4}$ several times until the cubic graph processes $n$ vertices. By Lemma 2.1.1 (i)(ii), $G^{\prime} \in\left\langle\mathbb{Z}_{5}\right\rangle$. Let $G=3 G^{\prime}$. Then $G \in\left\langle\mathcal{S} \mathbb{Z}_{5}\right\rangle$ by Theorem 2.1 .2 (i). Since $G^{\prime}$ is 3-edgeconnected, $G$ is a 9 -edge-connected strongly $\mathbb{Z}_{5}$-connected $d$-realization.

Case $2: d_{2} \geq 10$.

Thus $d_{1} \geq d_{2} \geq 10$, and we let $d^{\prime}=\left(d_{1}-1, d_{2}-1, d_{3}, \cdots, d_{n}\right)$. By Theorem 2.1.4, $d^{\prime}$ is multigraphic. By induction on $m, d^{\prime}$ has a 9-edge-connected strongly $\mathbb{Z}_{5}$-connected realization $G^{\prime}$. Construct the graph $G$ from $G^{\prime}$ by adding one edge joining $\left(d_{1}-1\right)$-vertex and $\left(d_{2}-1\right)$-vertex in graph $G^{\prime}$. By Lemma 2.1.1 (v), $G$ is also a 9-edge-connected strongly $\mathbb{Z}_{5}$-connected realization of $d$.

Now, we consider the remaining case.

Case $3: d_{1} \geq 10$ and $d_{2}=\cdots=d_{n}=9$.

If $d_{1} \geq 18$, we let $d^{\prime}=\left(d_{1}-9, d_{2}, \cdots, d_{n-1}\right)$. Then $d^{\prime}$ is multigraphic as $d_{1}-9 \leq \sum_{i=2}^{n-1} d_{i}$ and by Theorem 3.3.3. By induction on $m$, there exists a 9-edge-connected strongly $\mathbb{Z}_{5}$-connected graph $G^{\prime}$ as $d^{\prime}$-realization. Construct the graph $G$ by adding one new vertex $v_{n}$ and 9 parallel edges joining $v_{n}$ and $\left(d_{1}-9\right)$-vertex in $G^{\prime}$. By Lemma 2.1.1 (iii)(v), $G$ is the desired graph. Combining Case 1, we assume $10 \leq d_{1} \leq 17$ below.

Case 3.1: $d_{1}$ is odd.

Since $\sum_{i=1}^{n} d_{i}$ is even, $n$ is even and $n \geq 4$. If $n=4$ and $11 \leq d_{1} \leq 15$, we let $d_{1}-9=2 q$, where $1 \leq q \leq 3$. Let $v$ be an arbitrary vertex in $3 K_{4}$ and let $e_{1}, \cdots, e_{q}$ be non-parallel edges not adjacent to $v$ in $3 K_{4}$. We obtain the graph $G$ as $d$-realization from $3 K_{4}$ by inserting the edges $e_{1}, \cdots, e_{q}$ to the vertex $v$. By Lemma 2.1.5 (i), $G$ is 9-edge connected. Since $G$ contains $J_{2}$ as a spanning subgraph, by Lemma 2.1.7 and Lemma 2.1.1 (v), $G \in\left\langle\mathcal{S Z}_{5}\right\rangle$. Otherwise, $\left(d_{1}, d_{2}, d_{3}, d_{4}\right)=(17,9,9,9)$, which has already been handled in Lemma 2.2.3.

If $n \geq 6$, we obtain a graph $G^{\prime}$ by applying $Y-\Delta$ operation on $K_{4}$ repeatedly until the cubic graph processes $n$ vertices. Denote the last obtained vertex by $v_{1}$ in $G^{\prime}$, which is in the last 
generated triangle. Let $d_{1}-9=2 q$, where $1 \leq q \leq 4$. We select $q$ edges $e_{1}, \cdots, e_{q}$ that are coming from the edges of the basic graph $K_{4}$, which are not adjacent to $v_{1}$ in the graph $G^{\prime}$. Obtain the graph $G$ from $3 G^{\prime}$ by inserting the edges $e_{1}, \cdots, e_{q}$ to $v_{1}$. By Lemma 2.1.5 (i), $G$ is 9 -edge-connected. To verify that $G$ is strongly $\mathbb{Z}_{5}$-connected, we first observe that the graph induced by the vertices of the last generated triangle is strongly $\mathbb{Z}_{5}$-connected as it contains $J_{1}$ as a spanning subgraph. Then we can contract the last generated triangle and consecutively contract all the generated triangles, the remaining graph is strongly $\mathbb{Z}_{5}$-connected as it contains a $J_{2}$ as a spanning subgraph. By Lemma 2.1.1 (v), $G$ is a strongly $\mathbb{Z}_{5}$-connected $d$-realization.

Case 3.2: $d_{1}$ is even.

Since $\sum_{i=1}^{n} d_{i}$ is even, $n$ is odd and $n \geq 3$. When $n=3$, we have $d=\left(d_{1}, d_{2}, d_{3}\right)=\left(d_{1}, 9^{2}\right)$ and it is straightforward to obtain a 9-edge connected $d$-realization $G$ containing the graph $J_{1}$. If $n=5$ and $d_{1}=14$ or $d_{1}=16$ or $n=7$ and $d_{1}=16$, then the multigraphic sequences are $\left(14,9^{4}\right),\left(16,9^{4}\right),\left(16,9^{6}\right)$, which are all verified by Lemma 2.2.3.

The remaining cases are as follows: $n \geq 9$, or $n=7$ and $10 \leq d_{1} \leq 14$, or $n=5$ and $10 \leq d_{1} \leq 12$. We construct a graph $G^{\prime}$ by applying $Y-\Delta$ operation on $K_{4}$ repeatedly until the cubic graph processes $n-1$ vertices. Let $E^{\prime} \subset E\left(G^{\prime}\right)$ consist the edges of the base graph $K_{4}$ and one edge in each generated triangle in $G^{\prime}$. Thus $\left|E^{\prime}\right| \geq 8$ if $n \geq 9 ;\left|E^{\prime}\right|=7$ if $n=7 ;\left|E^{\prime}\right|=6$ if $n=5$. Let $d_{1}=2 q$. Note that $\left|E^{\prime}\right| \geq q$. We select the edges $e_{1}, \cdots, e_{q}$ in $E^{\prime}$ and obtain the graph $G$ from $3 G^{\prime}$ by inserting the edges $e_{1}, \cdots, e_{q} \in E^{\prime}$ to a new vertex $v_{1}$. By Lemma 2.1.5 (ii), $G$ is 9 -edge connected. Clearly, $G$ is a $d$-realization. To see that $G$ is strongly $\mathbb{Z}_{5}$-connected, we first recall that $J_{1}$ and $J_{2}$ are strongly $\mathbb{Z}_{5}$-connected by Lemma 2.1.7. By contracting $J_{1}$ and $3 K_{3}$ in the generated triangles of $G$ consecutively, the resulting graph consists of 5 vertices, namely $v_{1}$ and the remaining 4 vertices induced a graph containing $J_{2}$. We then contract $J_{2}$ and the resulting $2 q$ parallel edges to obtain $K_{1}$. This shows that $G$ is a strongly $\mathbb{Z}_{5}$-connected by Lemma 2.1.1 (v). The proof is completed.

Proof of Corollary 1.3.4: We assume that $d=\left(d_{1}, \cdots, d_{n}\right)$ is a nonincreasing multigraphic sequence with $d_{n} \geq 8$. By Theorem 3.3.3, $d_{1} \leq \sum_{i=2}^{n} d_{i}$. The case of $n=2$ is trivial. Assume $n \geq 3$. Suppose the contrary that $\left(d_{1}, \cdots, d_{n}\right)$ is a counterexample with $m=\sum_{i=1}^{n} d_{i}$ minimized.

If $d_{1} \geq 10$, let $d^{\prime}=\left(d_{1}^{\prime}, d_{2}^{\prime}, \cdots, d_{n}^{\prime}\right)=\left(d_{1}-2, d_{2}, \cdots, d_{n}\right)$. If $d_{1}-2=d_{1}^{\prime} \geq d_{2}^{\prime}=d_{2}$, then $d_{1}^{\prime} \leq d_{1} \leq \sum_{i=2}^{n} d_{i}=\sum_{i=2}^{n} d_{i}^{\prime}$. Otherwise, $d_{1}^{\prime}=d_{1}-2<d_{2}^{\prime}$, then $\max _{i \in[n]}\left\{d_{i}^{\prime}\right\}=d_{2} \leq d_{1} \leq$ $d_{1}-2+\sum_{i=3}^{n} d_{i}=d_{1}^{\prime}+\sum_{i=3}^{n} d_{i}^{\prime}$, since $n \geq 3$. Hence $d^{\prime}$ is multigraphic in any case by Theorem 3.3.3. Let $G^{\prime}$ be a 8-edge-connected modulo 5-orientation $d^{\prime}$-realization by the minimality. We obtain the desired graph $G$ from $G^{\prime}$ by inserting one edge to the $\left(d_{1}-2\right)$-vertex in $G^{\prime}$. By Lemma 2.1.5 (i), $G$ is also a 8-edge-connected modulo 5-orientation $d$-realization, a contradiction. 
If $d_{1}=8$, then $d_{1}=\cdots=d_{n}=8$. Hence $G=4 C_{n}$ is a 8-edge-connected modulo 5orientation $d$-realization, a contradiction. Assume $d_{1}=9$ in the following. As $\sum_{i=1}^{n} d_{i}$ is even, we have $d_{2}=9$. If $d_{n}=8$, we let $d^{\prime}=\left(d_{1}^{\prime}, d_{2}^{\prime}, \cdots, d_{n-1}^{\prime}\right)=\left(d_{1}, d_{2}, \cdots, d_{n-1}\right)$. Then $d_{1}^{\prime} \leq d_{2}^{\prime} \leq \sum_{i=2}^{n} d_{i}^{\prime}$, and so $d^{\prime}$ is multigraphic by Theorem 3.3.3. Let $G^{\prime}$ be a 8-edge-connected modulo 5 -orientation $d^{\prime}$-realization by the minimality. Let $e_{i} \in E\left(G^{\prime}\right), 1 \leq i \leq 4$. We obtain the desired graph $G$ from $G^{\prime}$ by inserting the edges $e_{1}, \cdots, e_{4}$ to one new vertex $v_{n}$. By Lemma 2.1.5 (ii), $G$ is a 8-edge-connected modulo 5-orientation realization of $d$, a contradiction. Therefore, we have $d_{n} \geq 9$, and it follows from Theorem 1.3.3 that there exists a 9-edge-connected strongly $\mathbb{Z}_{5}$-connected graph $G$ as a $d$-realization, which admits a modulo 5 -orientation as well. This contradiction completes the proof of Corollary 1.3.4. 


\section{Chapter 3}

\section{Modulo Orientations and Matchings in Graphs}

This chapter includes joint work with Han, Lai and Li, see [55].

\subsection{Introduction}

Let $t$ be a positive integer and let $\left\langle\mathcal{S} \mathbb{Z}_{2 t+1}\right\rangle$ be the graph family consisting of all strongly $\mathbb{Z}_{2 t+1^{-}}$ connected graphs.

Tutte [86] showed that a planar graph $H$ has a proper vertex 3-coloring if only if its planar dual $H^{*}$ admits a 3 -NZF (or equivalently $H^{*} \in \mathcal{M}_{3}$ ). The 3 -vertex-coloring planar graph problem is NP-complete, and thus the modulo 3-orientation problem is NP-complete by duality. In [64], MacGillivray and Siggers further proved that the homomorphism problem to odd cycle $C_{2 t+1}$ on planar graph is NP-complete. By the duality of circular flow and circular coloring, this also gives the NP-completeness of modulo $(2 t+1)$-orientation problem for fix $t>0$.

On the other hand, every triangle-free planar graph is vertex 3-colorable from the classical Grötzsch's 3-coloring theorem, which equivalently provides a 3-NZF for every 4-edge-connected planar graph by duality. Bill Tutte in 1970s suggested that the later statement maybe hold for nonplanar graphs as well. Tutte's Flow Conjectures were further extended by Jaeger [34] who conjectured that every $4 t$-edge-connected graph admits a modulo $(2 t+1)$-orientation, and by Lai [42] who conjectured that every $(4 t+1)$-edge-connected graph is strongly $\mathbb{Z}_{2 t+1}$-connected. In 2012, Thomassen [77] proved the weak versions of these conjectures for high edge-connectivity $2(2 t+1)^{2}+2 t+1$. It was further improved to $6 t$-edge-connected graphs by Lovász et al. [60].

Theorem 3.1.1. (Lovász, Thomassen, Wu and Zhang [60]) Every 6t-edge-connected graph is strongly $\mathbb{Z}_{2 t+1}$-connected, and therefore admits a modulo $(2 t+1)$-orientation. 
However, both conjectures of Jaeger [34] and Lai [42] were disproved in [31] recently, for larger values of $t$.

Theorem 3.1.2. ( [31])

(1) For every integer $t \geq 3$, there exist infinitely many 4t-edge-connected graphs without a modulo $(2 t+1)$-orientation.

(2) For every integer $t \geq 5$, there exist infinitely many $(4 t+1)$-edge-connected graphs without a modulo $(2 t+1)$-orientation.

Pushing further on the edge connectivity condition to warrant modulo $(2 t+1)$-orientation seems to be very challenge in either direction. It remains widely open to seek other types of nice sufficient condition for modulo orientations.

In this paper, we prove a relatively positive result that if a graph family has bounded matching number, then after certain reduction operations, there are only finitely many $(2 t+2)$ edge-connected graphs without modulo $(2 t+1)$-orientation in this family. To state our theorem formally, we shall first introduce the concept of $\left\langle\mathcal{S} \mathbb{Z}_{2 t+1}\right\rangle$-reduction below.

Fixed a graph $\Gamma$, each vertex contains in a maximal strongly $\mathbb{Z}_{2 t+1}$-connected subgraph, since the singleton $K_{1} \in\left\langle\mathcal{S} \mathbb{Z}_{2 t+1}\right\rangle$. Select all the maximal strongly $\mathbb{Z}_{2 t+1}$-connected subgraph of $\Gamma$, denoted by $G_{1}, G_{2}, \cdots, G_{c}$. Define $\Gamma^{\prime}=\Gamma /\left(\cup_{i=1}^{c} E\left(G_{i}\right)\right)$ as the $\left\langle\mathcal{S} \mathbb{Z}_{2 t+1}\right\rangle$-reduction of $\Gamma$, or saying that $\Gamma$ is $\left\langle\mathcal{S} \mathbb{Z}_{2 t+1}\right\rangle$-reduced to $\Gamma^{\prime}$. A graph $\Gamma$ is called $\left\langle\mathcal{S} \mathbb{Z}_{2 t+1}\right\rangle$-reduced if $\Gamma=\Gamma^{\prime}$ (i.e. it contains no strongly $\mathbb{Z}_{2 t+1}$-connected subgraph $G$ with $|V(G)|>1$ ). The construction in [31] in fact indicates that for every $t \geq 5$, there are infinitely many $\left\langle\mathcal{S} \mathbb{Z}_{2 t+1}\right\rangle$-reduced graphs without a modulo $(2 t+1)$-orientation in the family of all $(4 t+1)$-edge-connected graphs. For $G \in\left\langle\mathcal{S} \mathbb{Z}_{2 t+1}\right\rangle$, it is proved in [42] that, for every supergraph $\Gamma$ of $G, \Gamma \in \mathcal{M}_{2 t+1}$ if and only if $G / H$ does. Therefore, seeking modulo $(2 t+1)$-orientations of a graph $\Gamma$ is equivalent to seeking modulo $(2 t+1)$-orientations of the $\left\langle\mathcal{S} \mathbb{Z}_{2 t+1}\right\rangle$-reduction of $\Gamma$.

Our first main result is formally stated as follows.

Theorem 3.1.3. For fixed integer $s>0$, there exists a graph family $\mathcal{G}(t, s)$ of finite cardinality such that every graph $\Gamma$ with $\kappa^{\prime}(\Gamma) \geq 2 t+2$ and $\alpha^{\prime}(\Gamma) \leq s$ has a modulo $(2 t+1)$-orientation if and only if its $\left\langle\mathcal{S} \mathbb{Z}_{2 t+1}\right\rangle$-reduction $\Gamma^{\prime} \notin \mathcal{G}(t, s)$.

As the $\left\langle\mathcal{S} \mathbb{Z}_{2 t+1}\right\rangle$-reduction operation is a special type of contraction, Theorem 3.1.3 is still valid with contraction replacing $\left\langle\mathcal{S} \mathbb{Z}_{2 t+1}\right\rangle$-reduction. That is, for every graph $\Gamma$ with $\kappa^{\prime}(\Gamma) \geq$ $2 t+2$ and $\alpha^{\prime}(\Gamma) \leq s, \Gamma \in \mathcal{M}_{2 t+1}$ if and only if $\Gamma$ is not contractible to a graph in the finite graph family $\mathcal{G}(p, s)$.

The contractibility problem, asking to decide whether a graph is contractible to $G$, is NPcomplete for any triangle-free graph $G$ other than star [12], and it is polynomial-time solvable 
when $G$ is a star [12], a clique [52,69], and some other graphs. It is proved by Kamiński, Paulusma and Thilikos [40] that for any graph $G$, there is a polynomial-time algorithm to decide whether a planar graph is contractible to $G$. Since there are constant many graphs in the family $\mathcal{G}(t, s)$ in Theorem 5.3.4, we can check whether a planar $\Gamma$ is contractible to a member of $\mathcal{G}(t, s)$ in polynomial-time. Furthermore, each member of $\mathcal{G}(t, s)$ has size bounded by some constant $N(t, s)$ (see Section 2), which is constant-time determined. Therefore, we obtain a polynomialtime algorithm from Theorem 5.3.4 to decide whether a planar graph $\Gamma$ with $\kappa^{\prime}(\Gamma) \geq 2 t+2$ and $\alpha^{\prime}(\Gamma) \leq s$ has a modulo $(2 t+1)$-orientation for fixed $s$ and $t$. In contrast, the modulo orientation problem remains NP-complete for planar graph $G$ with $\kappa^{\prime}(G) \geq 2 t+2$ when $t \geq 2$ as shown by Esperet, Montassier, Ochem, Pinlou [24].

Corollary 3.1.4. For fixed integers $s>0$ and $t>0$, there exists a polynomial-time algorithm to decide whether a planar graph $\Gamma$ with $\kappa^{\prime}(\Gamma) \geq 2 t+2$ and $\alpha^{\prime}(\Gamma) \leq s$ has a modulo $(2 t+1)$ orientation.

Note that the polynomial-time algorithm of Corollary 3.1.4 extends to graphs embeddable on surfaces as well, since Kamiński et al. [40] also provides a polynomial-time algorithm for contractibility of graphs embeddable on a given surface.

Theorem 5.3.4, together with Theorem 3.1.1, immediately implies a Chvátal-Erdős type result: if $G$ satisfies a Chvátal-Erdős type condition $\kappa^{\prime}(G) \geq \max \left\{\alpha^{\prime}(G), 2 t+2\right\}$, then $G$ admits a modulo $(2 t+1)$-orientation with essentially finitely many exceptions.

Theorem 3.1.5. For fixed integer $t>0$, there is a finite family of non-modulo $(2 t+1)$ orientation-admissible graphs $\mathcal{F}_{1}(t)$ with the following property: A graph $\Gamma$ with $\kappa^{\prime}(\Gamma) \geq \max \left\{\alpha^{\prime}(G)\right.$, $2 t+2\}$ has a modulo $(2 t+1)$-orientation if and only if it is not contracted to a graph in $\mathcal{F}_{1}(t)$.

Theorems 5.3.4 and 3.1.5 are best possible results in a sense that the edge connectivity $2 t+2$ cannot be replaced by $2 t+1$. In fact, there are infinitely many $(2 t+1)$-edge-connected $\left\langle\mathcal{S} \mathbb{Z}_{2 t+1}\right\rangle$-reduced graphs with fixed matching number admitting no modulo $(2 t+1)$-orientation. Let $n \geq s \geq 3$ be integers. The graph $K_{s, n}^{+k}$ is constructed from complete bipartite graph $K_{s, n}$ by adding $k$ extra edges connecting vertices of degree $n$. We write $K_{s, n}^{+}$as an abbreviation of $K_{s, n}^{+1}$ in this paper. It is easy to observe that $K_{3, n}^{+}$admits no modulo 3 -orientation for any $n \geq 3$. This example can be generalized to modulo $(2 t+1)$-orientations for any $t$. For example, let $\mathcal{K}(t)$ be a graph family defined by $\mathcal{K}(t)=\left\{K_{2 t+1, c}^{+}: c \geq 2 t+1\right\}$. Then each member in $\mathcal{K}(t)$ is a $(2 t+1)$-edge-connected $\left\langle\mathcal{S} \mathbb{Z}_{2 t+1}\right\rangle$-reduced graph without a modulo $(2 t+1)$-orientation, providing infinitely many examples of connectivity $2 t+1$ respected to Theorems 5.3.4 and 3.1.5.

The 3-Flow Conjecture of Tutte has been intensively studied. For example, Grünbaum, and Steinburg and Younger proved the following, respectively. 
Theorem 3.1.6. (1) (Grünbaum [27]) Every bridgeless planar graph with at most three 3edge-cuts has a 3 -NZF.

(2) (Steinburg and Younger [75]) Every bridgeless projective planar graph with at most one 3-edge-cut has a 3-NZF.

While Theorems 5.3.4 and 3.1.5 with $p=1$ address the 3-flow problem, the next result of this research is an improvement to Theorem 3.1 .5 by characterizing all the (infinitely many) $\left\langle\mathcal{S} \mathbb{Z}_{3}\right\rangle$-reduced graphs $G$ with $\kappa^{\prime}(G) \geq \alpha^{\prime}(G)$. Denote $\mathcal{G}_{1}$ to be a class of graphs in Figure 3.1.
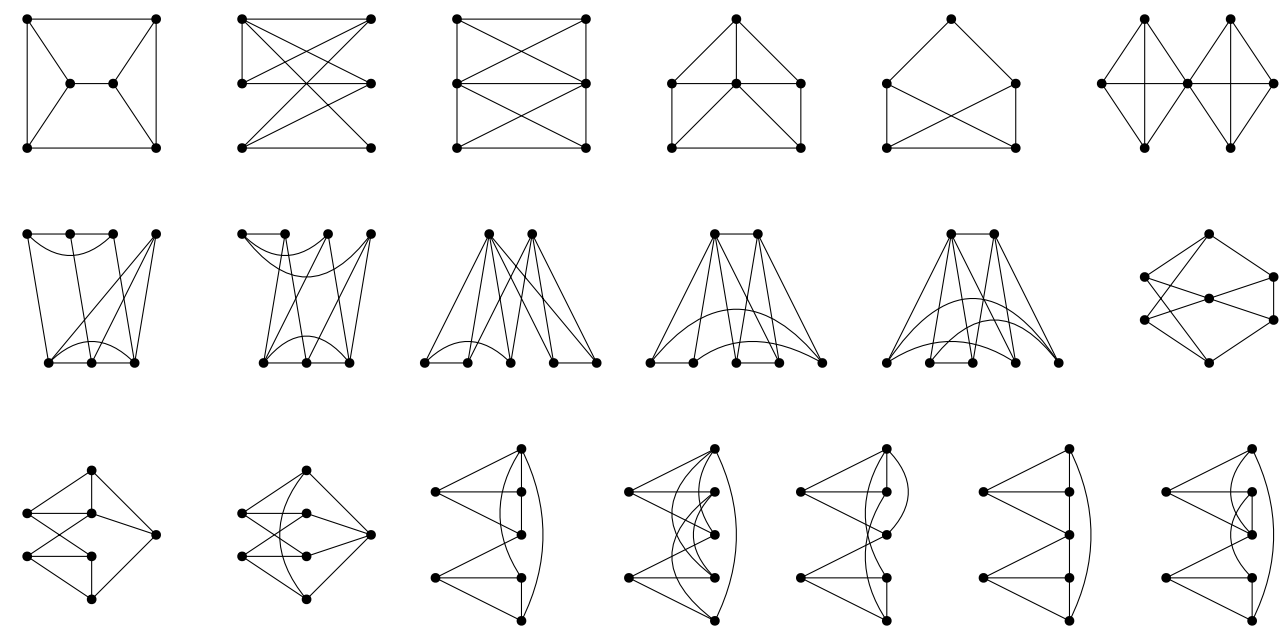

Figure 3.1: The graph family $\mathcal{G}_{1}$ in Theorem 3.1.7.

Theorem 3.1.7. Every bridgeless graph $G$ with $\kappa^{\prime}(G) \geq \alpha^{\prime}(G)$ has a $3-N Z F$, unless $G$ belongs to one of the following exceptional cases.

(1) $G$ can be contracted (or $\left\langle\mathcal{S} \mathbb{Z}_{3}\right\rangle$-reduced) to a graph in $\mathcal{G}_{1}$.

(2) $G$ is the graph $K_{4}$ or $K_{3, t}^{+}$for a positive integer $t \geq 3$.

In 2002, Kochol [39] showed that Tutte's 3-Flow Conjecture is equivalent to a seemly stronger form that every bridgeless graph with at most three 3 -edge-cuts admits a 3 -NZF. The corollary below is an immediate application of Theorem 3.1.7.

Corollary 3.1.8. Every bridgeless graph $G$ with $\kappa^{\prime}(G) \geq \alpha^{\prime}(G)$ admits a 3 -NZF provided that $G$ has at most three 3 -edge-cuts.

In the next two sections, we shall give the proofs of our main results, Theorems 5.3 .4 and 3.1.7. 


\subsection{Modulo Orientations and Matchings}

We start with some needed lemmas. Let $m H$ be the graph constructed from $H$ by replacing each edge of $H$ with $m$ parallel edges. We summarize some fundamental properties of modulo

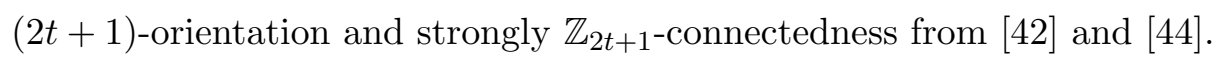

Lemma 3.2.1. ( $[42,44])$ For fixed integers $m, t>0$ and fixed graph $H$, we have properties below:

(1) For $H \in\left\langle\mathcal{S} \mathbb{Z}_{2 t+1}\right\rangle$ and $e \in E(H), H / e \in\left\langle\mathcal{S} \mathbb{Z}_{2 t+1}\right\rangle$.

(2) For $H \subseteq \Gamma$, if both $\Gamma / H \in\left\langle\mathcal{S} \mathbb{Z}_{2 t+1}\right\rangle$ and $H \in\left\langle\mathcal{S} \mathbb{Z}_{2 t+1}\right\rangle$, then $\Gamma \in\left\langle\mathcal{S} \mathbb{Z}_{2 t+1}\right\rangle$.

(3) The graph $m K_{2} \in\left\langle\mathcal{S} \mathbb{Z}_{2 t+1}\right\rangle$ if and only if $m \geq 2 t$.

(4) A nontrivial complete graph $K_{m} \in\left\langle\mathcal{S} \mathbb{Z}_{2 t+1}\right\rangle$ if and only if $m \geq 4 t+1$.

(5) The graph $H \in \mathcal{M}_{2 t+1}$ if and only if its $\left\langle\mathcal{S Z}_{2 t+1}\right\rangle$-reduction $H^{\prime} \in \mathcal{M}_{2 t+1}$.

(6) The graph $H \in\left\langle\mathcal{S} \mathbb{Z}_{2 t+1}\right\rangle$ if and only if its $\left\langle\mathcal{S} \mathbb{Z}_{2 t+1}\right\rangle$-reduction $H^{\prime}=K_{1}$.

The lifting lemma below can be easily obtained from the definition of strongly $\mathbb{Z}_{2 t+1^{-}}$ connected graphs.

Lemma 3.2.2 (Lifting). For a graph $H$ with $v_{1} v_{2}, v_{1} v_{3} \in E(H)$, construct a graph $H_{\left[v_{1}, v_{2} v_{3}\right]}$ from $H$ by removing $v_{1} v_{2}, v_{1} v_{3}$ and adding a new edge $v_{2} v_{3}$. If $H_{\left[v_{1}, v_{2} v_{3}\right]} \in\left\langle\mathcal{S} \mathbb{Z}_{2 t+1}\right\rangle$, then $H \in\left\langle\mathcal{S} \mathbb{Z}_{2 t+1}\right\rangle$.

For notational convenience, we always use $U=\left\{u_{1}, \ldots, u_{m}\right\}$ and $V=\left\{v_{1}, \ldots, v_{n}\right\}$ to denote the two parts of a complete bipartite graph $K_{m, n}$. For any subset $\left\{t_{1}, t_{2}, \ldots, t_{\ell}\right\}$ of $\mathbb{Z}_{n}$, form a graph $K\left(t_{1}, t_{2}, \ldots, t_{\ell}\right)$ from $K_{m, n}$ by identifying $v_{1}, \ldots, v_{t_{1}}$, identifying $v_{t_{i}+1}, \ldots, v_{t_{i+1}}$ for each $1 \leq i \leq \ell-1$ and identifying $v_{t_{\ell}+1}, \ldots, v_{n}$, respectively. Define a family of graphs $\mathcal{B}^{*}(m, n)$ to be

$$
\mathcal{B}^{*}(m, n)=\left\{K\left(t_{1}, t_{2}, \ldots, t_{\ell}\right):\left\{t_{1}, t_{2}, \ldots, t_{\ell}\right\} \subseteq \mathbb{Z}_{n}\right\}
$$

Lemma 3.2.3. Every member of $\mathcal{B}^{*}\left(2 p+2,4 p^{2}+2 p\right)$ is strongly $\mathbb{Z}_{2 p+1}$-connected.

Proof. We first apply the lifting lemma to show the complete bipartite graph $K_{2 p+2,4 p^{2}+2 p}$ is strongly $\mathbb{Z}_{2 p+1}$-connected. Recall that $U=\left\{u_{1}, u_{2} \ldots, u_{2 p+2}\right\}$ and $V=\left\{v_{1}, v_{2}, \ldots, v_{4 p^{2}+2 p}\right\}$ are the set of all degree $4 p^{2}+2 p$ vertices and all degree $2 p+2$ vertices in $K_{2 p+2,4 p^{2}+2 p}$, respectively. Obtain a graph $K^{\prime}$ from $K_{2 p+2,4 p^{2}+2 p}$ by lifting $v_{2 p i+1} u_{i+1}, v_{2 p i+1} u_{i+2}$, lifting $v_{2 p i+2} u_{i+1}, v_{2 p i+2} u_{i+2}, \ldots$, and lifting $v_{2 p i+2 p} u_{i+1}, v_{2 p i+2 p} u_{i+2}$ to obtain $2 p$ parallel edges between $u_{i+1}$ and $u_{i+2}$, for each $0 \leq i \leq 2 p$. Then $K^{\prime}[U] \in\left\langle\mathcal{S} \mathbb{Z}_{2 p+1}\right\rangle$ by Lemma 5.1.3(3). Notice 
that $\left|\left[v_{j}, U\right]_{K^{\prime}}\right|=2 p$ for each $1 \leq j \leq 4 p^{2}+2 p$. By Lemma 5.1.3(2)(3), we have $K^{\prime} \in\left\langle\mathcal{S} \mathbb{Z}_{2 p+1}\right\rangle$. Therefore, $K_{2 p+2,4 p^{2}+2 p} \in\left\langle\mathcal{S} \mathbb{Z}_{2 t+1}\right\rangle$ by Lemma 5.3.1. Since the strongly $\mathbb{Z}_{2 p+1}$-connectedness is preserved under identifying vertices and every member in $\mathcal{B}^{*}\left(2 p+2,4 p^{2}+2 p\right)$ is obtained from identifying vertices of $K_{2 p+2,4 p^{2}+2 p}$, we conclude that every member of $\mathcal{B}^{*}\left(2 p+2,4 p^{2}+2 p\right)$ is strongly $\mathbb{Z}_{2 p+1 \text {-connected. }}$

The following elementary counting lemma is also needed in our proof.

Lemma 3.2.4. For fixed positive integers $\ell, n$, there are exactly $\left(\begin{array}{c}n+\ell-1 \\ \ell-1\end{array}\right)$ non-negative integral solutions $\left.l x_{1}, x_{2}, \ldots, x_{\ell}\right\rangle$ for the equation $x_{1}+x_{2}+\cdots+x_{\ell}=n$.

Define $N(t, s)=\left(4 t^{2}+2 t\right)\left(\begin{array}{c}2 s+2 t+1 \\ 2 s-1\end{array}\right)+2 s$. Let $\mathcal{F}(t, s)$ be the family of all $(2 t+2)$-edgeconnected $\left\langle\mathcal{S} \mathbb{Z}_{2 t+1}\right\rangle$-reduced graphs of order between 2 and $N(t, s)$ with matching number at most $s$. Then the edge multiplicity of each graph in $\mathcal{F}(t, s)$ is at most $2 t-1$ by Lemma 5.1.3(3). So there are indeed finitely many graphs in $\mathcal{F}(t, s)$. Define $\mathcal{G}(t, s)$ as the family of graphs in $\mathcal{F}(t, s)$ which are not in $\mathcal{M}_{2 t+1}$. We will prove a stronger theorem below, which implies Theorem 5.3.4 by Lemma $5.1 .3(5)(6)$.

Theorem 3.2.5. For any $(2 t+2)$-edge-connected graph $\Gamma$ with $\alpha^{\prime}(\Gamma) \leq s$, we have $\Gamma \in\left\langle\mathcal{S} \mathbb{Z}_{2 t+1}\right\rangle$ if and only if $\Gamma$ cannot be $\left\langle\mathcal{S} \mathbb{Z}_{2 t+1}\right\rangle$-reduced to a member in $\mathcal{F}(t, s)$.

Proof. If $\Gamma \in\left\langle\mathcal{S} \mathbb{Z}_{2 t+1}\right\rangle$, then $\Gamma$ is $\left\langle\mathcal{S} \mathbb{Z}_{2 t+1}\right\rangle$-reduced to $K_{1} \notin \mathcal{F}(t, s)$ by Lemma 5.1.3(6). We shall show the converse that if $\Gamma$ cannot be $\left\langle\mathcal{S} \mathbb{Z}_{2 t+1}\right\rangle$-reduced to a member in $\mathcal{F}(t, s)$, then $\Gamma \in\left\langle\mathcal{S} \mathbb{Z}_{2 t+1}\right\rangle$.

Let $\Gamma$ be a counterexample and let $\Gamma^{\prime}$ be its $\left\langle\mathcal{S} \mathbb{Z}_{2 t+1}\right\rangle$-reduction. Then $\Gamma^{\prime} \notin \mathcal{F}(t, s)$ and this leads to

$$
\left|V\left(\Gamma^{\prime}\right)\right|>N(t, s)=\left(4 t^{2}+2 t\right)\left(\begin{array}{c}
2 s+2 t+1 \\
2 s-1
\end{array}\right)+2 s .
$$

Since $\Gamma^{\prime}$ is the $\left\langle\mathcal{S} \mathbb{Z}_{2 t+1}\right\rangle$-reduction of $\Gamma$, we have $\alpha^{\prime}\left(\Gamma^{\prime}\right) \leq \alpha^{\prime}(\Gamma) \leq s$. Let $M=\left\{w_{1} w_{2}, w_{3} w_{4}, \ldots\right.$, $\left.w_{2 d-1} w_{2 d}\right\}$ be a maximum matching of $\Gamma^{\prime}$, where $d \leq s$. Denote $W=\left\{w_{1}, \ldots, w_{2 d}\right\}$. Then $Z=V\left(\Gamma^{\prime}\right)-W$ is an independent set of $\Gamma^{\prime}$ by the maximality of $M$. Since $\kappa^{\prime}\left(\Gamma^{\prime}\right) \geq 2 t+2$, we have $\left|[z, W]_{\Gamma^{\prime}}\right| \geq 2 t+2$ for any $z \in Z$. Pick arbitrary $2 t+2$ edges from $[z, W]_{\Gamma^{\prime}}$, denoted by $H(z)$, for each $z \in Z$. Let $\Gamma_{1}^{\prime}=\cup_{z \in Z} H(z)$ be an edge subset as well as the edge-induced subgraph of $\Gamma^{\prime}$.

We claim that there exists a member of $\mathcal{B}^{*}\left(2 t+2,4 t^{2}+2 t\right)$ contained in $\Gamma_{1}^{\prime}$, therefore in $\Gamma^{\prime}$. This will contradict to the assumption that $\Gamma^{\prime}$ is a $\left\langle\mathcal{S} \mathbb{Z}_{2 t+1}\right\rangle$-reduced graph by Lemma 5.3.3.

For any $w \in W$ and $z \in Z$, we use $x(w, z)=\left|[w, z]_{\Gamma_{1}^{\prime}}\right|$ to denote the number of edges in $H(z)$ between $w$ and $z$. We also define $x(w, z)=0$ if $w$ is not in the graph $H(z)$. Since $H(z)$ consists of $2 t+2$ edges, we have, for each $z \in Z$,

$$
x\left(w_{1}, z\right)+x\left(w_{2}, z\right)+\cdots+x\left(w_{2 d}, z\right)=2 t+2 .
$$


Since $d \leq s$ and by (5.10), we have

$$
|Z|=\left|V\left(\Gamma^{\prime}\right)\right|-2 d>N(t, s)-2 s \geq\left(4 t^{2}+2 t\right)\left(\begin{array}{c}
2 s+2 t+1 \\
2 s-1
\end{array}\right) .
$$

It follows from Lemma 5.3.5 and Pigeon-Hole Principle that there exists a subset $Z_{1} \subset Z$ of size $4 t^{2}+2 t$ such that, for any $a, b \in Z_{1}$,

$$
\left.\left.l x\left(w_{1}, a\right), x\left(w_{2}, a\right), \ldots, x\left(w_{2 d}, a\right)\right\rangle=l x\left(w_{1}, b\right), x\left(w_{2}, b\right), \ldots, x\left(w_{2 d}, b\right)\right\rangle .
$$

Denote $x_{1}, \ldots, x_{\ell+1}$ to be all the nonzero coordinates in $\left.l x\left(w_{1}, a\right), x\left(w_{2}, a\right), \ldots, x\left(w_{2 d}, a\right)\right\rangle$. Then the graph $\left[S_{1}, Y\right]_{\Gamma_{1}^{\prime}} \cong K\left(t_{1}, t_{2}, \ldots, t_{\ell}\right)$ is a member of $\mathcal{B}^{*}\left(2 t+2,4 t^{2}+2 t\right)$, where $t_{1}=x_{1}$, $t_{\ell+1}=(2 t+2)-t_{\ell}$ and $t_{i}-t_{i-1}=x_{i}$ for $2 \leq i \leq \ell$. This proves the claim as well as the theorem.

In the following section, we will focus on the special important case of $t=1$, which is Theorem 3.1.7 concerning modulo 3-orientation. However, Theorem 3.1.7 seems not possible to extend to general modulo $(2 t+1)$-orientations. One may obverse that for $k \leq t$ and $c \geq 2 t+2$,

the graph $K_{2 t+1, c}^{+k}$ and some graphs obtained by identifying some large degree vertices of $K_{2 t+1, c}^{+k}$ are still $(2 t+1)$-edge-connected $\left\langle\mathcal{S} \mathbb{Z}_{2 t+1}\right\rangle$-reduced graphs without modulo $(2 t+1)$-orientations. Also, applying 2-sum operations on some of those graphs results more $\left\langle\mathcal{S} \mathbb{Z}_{2 t+1}\right\rangle$-reduced graphs without modulo $(2 t+1)$-orientations and with small matching number. Thus the structures of all such exceptional graphs are much more complicated, which seems far from being characterized. As we can see from the proof of Theorem 3.1.7 below, the arguments require to characterize all $\left.l \mathcal{S} \mathbb{Z}_{3}\right\rangle$-reduced graphs of small order. However, it seems hopeless to characterize all $\left.l \mathcal{S} \mathbb{Z}_{2 t+1}\right\rangle$ reduced graphs without modulo $(2 t+1)$-orientations on at most $4 t+3$ vertices by hand for general $t$.

\subsection{Proof of Theorem 3.1.7}

Note that a graph $G$ admits a modulo 3-orientation if and only if $G$ admits a 3-NZF; and the concept of strongly $\mathbb{Z}_{3}$-connected is the same as the so-called " $\mathbb{Z}_{3}$-connected" in some literature $[19,51]$. In this section we characterize all the exceptions of Theorem 5.3.4 when $t=1$. First, we display some more needed lemmas.

Lemma 3.3.1. ( [51]) If $G$ is $\left.l \mathcal{S} \mathbb{Z}_{3}\right\rangle$-reduced and $|V(G)|=7$, then $|E(G)| \leq 13$.

Lemma 3.3.2. ( $[51])$ If $\kappa^{\prime}(G) \geq 4$ and $|V(G)| \leq 13$, then $G$ admits a $3-N Z F$.

Lemma 3.3.3. (Hakimi [29]) Given a graph $G$, let $\ell: V(G) \rightarrow \mathbb{Z}$ be a function with $\sum_{u \in V(G)}=0$ and $\ell(u) \equiv d_{G}(u)(\bmod 2)$ for any $u \in V(G)$. Then $G$ admits an orientation $D$ with $\ell(u)=$ 
$d_{D}^{+}(u)-d_{D}^{-}(u)$ for any $u \in V(G)$ if and only if

$$
\left|\sum_{u \in A} \ell(u)\right| \leq\left|\partial_{G}(A)\right| \text { for any vertex subset } A \subseteq V(G) .
$$

The $k$-wheel $W_{k}$ is a graph constructed by adding a new center vertex connecting to each vertex of the $k$-cycle.

Lemma 3.3.4. ( [19]) For any integer $s \geq 1$, the even wheel $W_{2 s}$ is strongly $\mathbb{Z}_{3}$-connected.

Lemma 3.3.5. ( [51]) Assume that an odd wheel $W_{2 s+1}$ is a proper subgraph of a graph $\Gamma$. Let $A, B$ be a bipartition of the vertex set $V\left(W_{2 s+1}\right)$. Form a graph $\Gamma_{[A, B]}$ from $\Gamma$ by removing all edges of $E\left(W_{2 k+1}\right)$, contracting the sets $A$ and $B$ into two single vertices $u$ and $v$, respectively, and then connecting a new edge uv.

(i) If $\Gamma_{[A, B]}$ has a $3-N Z F$, then so does $\Gamma$.

(ii) If $\Gamma_{[A, B]} \in\left\langle\mathcal{S} \mathbb{Z}_{3}\right\rangle$, then $\Gamma \in\left\langle\mathcal{S} \mathbb{Z}_{3}\right\rangle$.

Lemma 3.3.6. Let $k \geq 0$ and $n \geq 3$ be integers. Then $K_{3, n}^{+k}$ admits a $3-N Z F$ if and only if $k \neq 1$.

Proof. First, assume $k \neq 1$ and we shall show $K_{3, n}^{+k}$ admits a 3 -NZF. If $k=0$, then $K_{3, n}^{+k}=K_{3, n}$ is a complete bipartite graph which obviously has a 3 -NZF. Now assume $k>0$. Let $V\left(K_{3, n}^{+k}\right)=$ $\left\{v_{1}, \ldots, v_{n+3}\right\}$, and denote the three vertices with degree more than 3 to be $v_{1}, v_{2}, v_{3}$. Since $\left.2 K_{2} \in l \mathcal{S} \mathbb{Z}_{3}\right\rangle$ by Lemma $5.1 .3(3)$, if $K_{3, n}^{+k}$ has parallel edges, then $\left.K_{3, n}^{+k} \in l \mathcal{S} \mathbb{Z}_{3}\right\rangle$ after contracting all 2-cycles by Lemma 5.1.3(2). Hence $K_{3, t}^{+k}$ admits a 3-NZF if $k \geq 4$. Now it just needs to show the cases of $k=2,3$, where the new added edges are non-parallel. If $k=3$, then edges of $K_{3, n}^{+3}$ can be partitioned to $K_{3, n}$ and $K_{3}$, which both have a 3 -NZF and so does $K_{3, n}^{+3}$. Thus assume $k=2$ in the following. Define a function $\ell: V\left(K_{3, n}\right) \rightarrow \mathbb{Z}$ by $\ell\left(v_{i}\right)$ are 3 and -3 alternately for $i \in\{4, \ldots, n+3\}, \ell\left(v_{2}\right)=-3, \ell\left(v_{1}\right)=\ell\left(v_{3}\right)=0$ when $n$ is odd and $\ell\left(v_{2}\right)=0, \ell\left(v_{1}\right)=3$, $\ell\left(v_{3}\right)=-3$ when $n$ is even. Then we can verify statement (3.2) of Lemma 3.3.3 for $K_{3, n}^{+k}$, and so $K_{3, t}^{+k}$ admits a modulo 3 -orientation $D$, or equivalently it admits 3 -NZF. Conversely, if $k=1$, then it is routine to check that $K_{3, n}^{+}$does not admit a $3-\mathrm{NZF}$.

Fan and Zhou [25] in 2008 characterized 3-NZF of simple graphs under Ore-condition.

Theorem 3.3.7. (Fan and Zhou [25]) Given a simple graph $G$ with $|V(G)| \geq 3$, if $G$ satisfies the Ore-condition that $d(s)+d(t) \geq|V(G)|$ for any pair of non-adjacent vertices $s, t$, then $G$ admits a 3-NZF if and only if $G$ is not isomorphic to a graph in Figure 3.2.

For a matching $M$ of a graph $G$, a path $P$ is called an $M$-augmenting path if both end vertices of $P$ are not in $V(M)$, and the edges of $P$ are alternately in $E(G)-M$ and in $M$. It is well-known that a matching $M$ is maximum if and only if there is no $M$-augmenting path. 


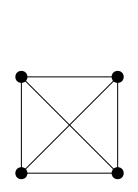

(a)

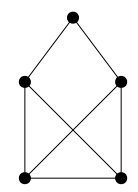

(b)

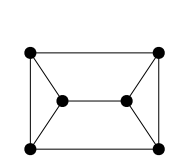

(c)

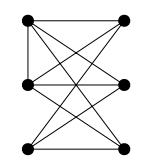

(d)

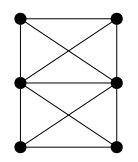

(e)

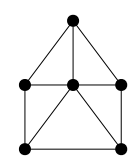

(f)

Figure 3.2: Graphs in Theorem 3.3.7.

Lemma 3.3.8. If a simple graph $G$ satisfies $|V(G)| \geq 3 k$ and $\kappa^{\prime}(G) \geq \alpha^{\prime}(G)=k$, then $G$ contains $K_{k, k}$ as a subgraph.

Proof. Let $V(G)=\left\{v_{1}, \ldots, v_{n}\right\}$. Since $\alpha^{\prime}(G)=k$, we may assume that $M=\left\{v_{2 i-1} v_{2 i}: 1 \leq\right.$ $i \leq k\}$ is a maximum matching of $G$. Hence there is no $M$-augmenting path in $G$, and moreover, $\left\{v_{2 k+1}, \ldots, v_{n}\right\}$ is an independent vertex-set. Assume that $v_{2 k+1}$ is adjacent to both end vertices of an edge of $M$, say $v_{2 k+1} \sim v_{1}$ and $v_{2 k+1} \sim v_{2}$ without loss of any generality. Then each of $v_{2 k+2}, \ldots, v_{n}$ is adjacent to neither $v_{1}$ nor $v_{2}$. Otherwise, it would cause an $M$-augmenting path, a contradiction. Since $\kappa^{\prime}\left(G^{\prime}\right) \geq k$, we know degree of $v_{2 k+1}$ is at least $k$, and so $v_{2 k+1}$ is also adjacent to both end vertices of another edge of $M$, say $v_{2 k+2} \sim v_{3}$ and $v_{2 k+2} \sim v_{4}$ without loss of any generality. Then each of $v_{2 k+3}, \ldots, v_{n}$ is adjacent to neither $v_{3}$ nor $v_{4}$ for the same reason. Repeat this argument again and again, we would have that $v_{2 k+i}$ is not adjacent to $v_{1}, v_{2}, \ldots, v_{2 i-1}, v_{2 i}$ for any $1 \leq i \leq k$. But this implies $v_{3 k}$ is adjacent to none of the vertices in $V(M)$. Since $\left\{v_{2 k+1}, \ldots, v_{n}\right\}$ is an independent vertex set, this shows that $v_{3 k}$ is an isolated vertex, contradicting to $\kappa^{\prime}\left(G^{\prime}\right) \geq k$.

Now we assume instead that $v_{2 k+1}$ is adjacent to precisely one end vertex of each edge of $M$, say $v_{2 k+1} \sim v_{2 i-1}$ for each $1 \leq i \leq k$. As there is no $M$-augmenting path, for any $j \geq 2, v_{2 k+j}$ is adjacent to none of $\left\{v_{2}, v_{4}, \ldots, v_{2 k}\right\}$. Since $\kappa^{\prime}\left(G^{\prime}\right) \geq k, v_{2 k+j}$ must be adjacent to $v_{2 i-1}$ for any $1 \leq i, j \leq k$. Therefore, the set $\left\{v_{1}, v_{3}, \ldots, v_{2 k-1}, v_{2 k+1}, \ldots, v_{2 k+k}\right\}$ induces a complete bipartite graph $K_{k, k}$ as required.

Now we shall prove Theorem 3.1.7, restated as the following equivalent version.

Theorem 3.3.9. Let $G$ be a bridgeless graph with $\kappa^{\prime}(G) \geq \alpha^{\prime}(G)$. Then either $G$ has a 3 -NZF, or $G$ can be $\left\langle\mathcal{S} \mathbb{Z}_{3}\right\rangle$-reduced to a graph in $\mathcal{G}_{1} \cup K_{4} \cup\left\{K_{3, t}^{+}: t \geq 3\right\}$.

Proof. When $\alpha^{\prime}(G)=1$, the simplification of $G$ is spanned by a $K_{1, n-1}$. As $G$ is bridgeless, it implies that $G$ consists of a branch of parallel edges joining to the center vertex. Hence by Lemma 5.1.3 $G$ has a 3-NZF. If $\alpha^{\prime}(G) \geq 6$, then by Theorem 3.1.1 $G$ admits a 3-NZF. It remains to show the cases of $2 \leq \alpha^{\prime}(G) \leq 5$. We use $G^{\prime}$ to represent the $\left\langle\mathcal{S} \mathbb{Z}_{3}\right\rangle$-reduction of $G$ as above. Then we have

$$
5 \geq \kappa^{\prime}\left(G^{\prime}\right) \geq \kappa^{\prime}(G) \geq \alpha^{\prime}(G) \geq \alpha^{\prime}\left(G^{\prime}\right) \geq 2 .
$$


Claim 3.3.1. If $G^{\prime} \notin \mathcal{M}_{3}$ and $\alpha^{\prime}\left(G^{\prime}\right)=2$, then $G^{\prime}$ is $K_{4}$ or (a) in Figure 3.3.

Proof of Claim 3.3.1. When $\left|V\left(G^{\prime}\right)\right|=4$, we have that $G^{\prime}$ is either $C_{4}$ or $C_{4}$ adding chords. They all have a 3-NZF except $K_{4}$. If $\left|V\left(G^{\prime}\right)\right|=5$, then $G^{\prime}$ is one of $K_{2,3}, C_{5}$ and $C_{5}$ adding chords. It is easy to verify they all have a 3 -NZF except the graph (a) in Figure 3.3. Next assume that $\left|V\left(G^{\prime}\right)\right|=n \geq 6$. Since $\alpha\left(G^{\prime}\right)=2$ and $\kappa^{\prime}\left(G^{\prime}\right) \geq 2$, we get that $G^{\prime}$ contains $K_{2,2}$ as a subgraph by Lemma 3.3.8. Assume a maximal matching of $G$ is $M=\left\{v_{1} v_{2}, v_{3} v_{4}\right\}$. Then $v_{5}, \ldots, v_{n}$ induce an independent set. Using a similar argument, one can justify that $G^{\prime} \cong K_{2, n-2}$ when $v_{1} \nsim v_{3}$ and $G^{\prime} \cong K_{2, n-2}^{+}$when $v_{1} \sim v_{3}$. In either case, $G^{\prime}$ has a 3 -NZF.

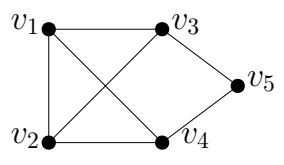

(a)

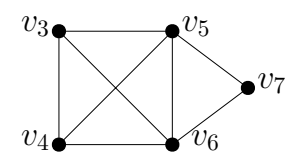

(b)

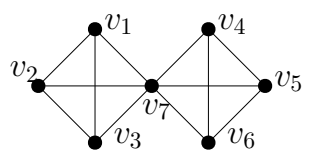

(c)

Figure 3.3: Graphs for Claims 3.3.1 and 3.3.5.

Claim 3.3.2. If $\alpha^{\prime}\left(G^{\prime}\right)=4,5$, then $G$ admits a $3-N Z F$.

Proof of Claim 3.3.2. Since $\kappa^{\prime}\left(G^{\prime}\right) \geq \alpha^{\prime}\left(G^{\prime}\right) \geq 4$, one has $\left|V\left(G^{\prime}\right)\right|=n \geq 14$ by Lemma 3.3.2. By Lemma 3.3.8, $G^{\prime}$ contains a subgraph which is isomorphic to $K_{4,4}$. Since $K_{4,4}$ is $\mathbb{Z}_{3}$-connected, this is a contradiction to $G^{\prime}$ is $\left\langle\mathcal{S} \mathbb{Z}_{3}\right\rangle$-reduced.

Claim 3.3.3. If $G^{\prime} \notin \mathcal{M}_{3}$ and $\left|V\left(G^{\prime}\right)\right| \geq 8$, then $G^{\prime} \cong K_{3, t}^{+}$for some integer $t \geq 5$.

Proof of Claim 3.3.3. From the above claims, one has $\alpha^{\prime}\left(G^{\prime}\right)=3$. Assume that $v_{6} \sim v_{5}, v_{4} \sim v_{3}$ and $v_{2} \sim v_{1}$, and the rest vertices form an independent set. Suppose that $v_{7}$ is adjacent to both $v_{2}$ and $v_{1}$. As $\kappa\left(G^{\prime}\right) \geq 3$, the degree of $v_{7}$ is at least 3 , and so, with out loss of generality, assume that $v_{7} \sim v_{3}$. Then $v_{8}$ is not adjacent to $v_{1}, v_{2}, v_{4}$ as $\alpha^{\prime}\left(G^{\prime}\right)=3$. Hence $v_{8}$ is adjacent to $v_{3}, v_{5}, v_{6}$. Since $v_{4}$ has degree at least 3 and cannot be adjacent to $v_{9}, \ldots, v_{n}$, we have that $v_{4}$ is adjacent to at least two of $v_{1}, v_{2}, v_{5}, v_{6}$. But in each case it results that $\alpha^{\prime}\left(G^{\prime}\right)>3$. So, for each $k \in\{7, \ldots, n\}, v_{k}$ is adjacent to each of $v_{5}, v_{3}, v_{1}$ and is adjacent to none of $v_{6}, v_{4}, v_{2}$. Thus $G^{\prime}$ must be one of the graphs $K_{3, n-3}, K_{3, n-3}^{+}, K_{3, n-3}^{+2}$ or $K_{3, n-3}^{+3}$. Among them, only the graph $K_{3, n-3}^{+}$does not have a 3 -NZF by Lemma 3.3.6.

Claim 3.3.4. If $G^{\prime} \notin \mathcal{M}_{3}$ and $\left|V\left(G^{\prime}\right)\right|=6$, then $G^{\prime}$ is one of (c), (d), (e) and (f) in Figure 3.2.

Proof of Claim 3.3.4. Since $\left|V\left(G^{\prime}\right)\right|=6$ and $\delta\left(G^{\prime}\right) \geq \kappa^{\prime}\left(G^{\prime}\right) \geq 3$, it satisfies the Ore-condition as in Theorem 3.3.7, and so $G^{\prime}$ is one of (c), (d), (e) and (f) in Figure 3.2.

A vertex is called a $k$-vertex if it is a vertex of degree $k$.

Claim 3.3.5. If $G^{\prime} \notin \mathcal{M}_{3}$ and $\left|V\left(G^{\prime}\right)\right|=7$, then $G^{\prime}$ is one of the following graphs: (c) in Figure 3.3; (e), (f) in Figure 3.4; (a), (c), (e) in Figure 3.5; (a), (b), (d)-(j) in Figure 3.6; or $K_{3,4}^{+}$. 
Proof of Claim 3.3.5. Note that $\alpha^{\prime}\left(G^{\prime}\right)=3$ by $\left|V\left(G^{\prime}\right)\right|=7$. First, assume that $G^{\prime}$ contains a 6 -vertex, say $v_{7}$. Let $H=G\left[\left\{v_{1}, \ldots, v_{6}\right\}\right]$ be an induced subgraph of $G$. As $\kappa^{\prime}\left(G^{\prime}\right) \geq \alpha^{\prime}\left(G^{\prime}\right)=3$, one has that the degree of each vertex of $H$ is at least 2. Thus $H$ contains a cycle. If it has an even length circle, then $G^{\prime}$ has a subgraph which is an even wheel graph. However, the even wheel graph is strongly $\mathbb{Z}_{3}$-connected by Lemma 3.3.4, which contradicts to $G^{\prime}$ is $\left\langle S \mathbb{Z}_{3}\right\rangle$-reduced. So $H$ has two circles of length 3 or one circle of length 5 . In the latter case, one can find an even length cycle in $H$ as well. Thus $H$ must have two circles of length 3 . We may assume that $v_{1}, v_{2}, v_{3}, v_{7}$ induce a $K_{4}$, say $H_{1}$; and $v_{4}, v_{5}, v_{6}, v_{7}$ induce the other $K_{4}$, say $H_{2}$. If there exists no edge between $H_{1}$ and $H_{2}$, then $G^{\prime}$ is exactly (c) in Figure 3.3. Clearly, this graph does not have a 3-NZF. If there exist edges between $H_{1}$ and $H_{2}$, then apply Lemma 3.3.5 to contract these two $K_{4}$ 's into a $K_{2}$, and the resulting graph is bridgeless with 3 vertices, which admits a 3-NZF. This implies $G$ has a 3-NZF by Lemma 3.3.5.

Now assume instead, $G^{\prime}$ does not have any 6 -vertex in the following. By Ore-condition, there exists a pair of nonadjacent 3-vertices; otherwise $G^{\prime}$ admits a 3-NZF by Theorem 3.3.7. We have 5 cases depending on the number of 3 -vertices.

(1) There are exactly two 3 -vertices. Assume $G^{\prime}$ has a 5 -vertex, then it has even number of 5 -vertices, and so $\left|E\left(G^{\prime}\right)\right| \geq(2 \cdot 5+2 \cdot 3+3 \cdot 4) / 2=14$. It follows by Lemma 3.3 .1 that $G^{\prime}$ is not $\left\langle S \mathbb{Z}_{3}\right\rangle$-reduced, a contradiction. Hence $G^{\prime}$ has no 5-vertex, namely, $G^{\prime}$ has five 4 -vertices. Next we apply lifting operations on those 4 -vertices. First split each 4 -vertex into two 2-vertices, and then shrink the corresponding 2-vertices. After splitting all the 4 -vertices, $G^{\prime}$ becomes a $3 K_{2}$, which has a 3 -NZF. Thus $G^{\prime}$ admits a $3-\mathrm{NZF}$.

(2) There are exactly three 3 -vertices. Thus there are odd number of 5 -vertices. If $G^{\prime}$ has more than one 5 -vertices, then we also have $\left|E\left(G^{\prime}\right)\right| \geq(3 \cdot 3+3 \cdot 5+4) / 2=14$. Thus $G^{\prime}$ is not a $\left\langle S \mathbb{Z}_{3}\right\rangle$-reduced graph by lemma 3.3.1, a contradiction. Then $G^{\prime}$ has exactly one 5 -vertex and three 4 -vertices. After splitting all 4-vertices as before, one can get a graph of order 4 with parallel edges, which has a $3-\mathrm{NZF}$. So $G^{\prime}$ also admits a $3-\mathrm{NZF}$.

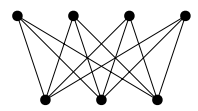

(a)

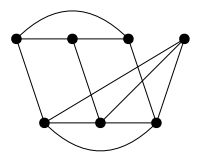

(e)

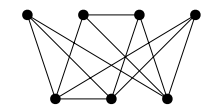

(b)

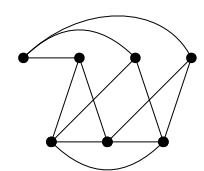

(f)

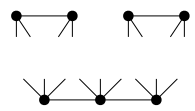

(c)

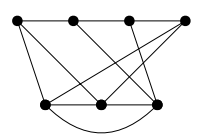

(g)

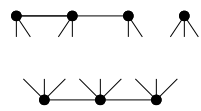

(d)

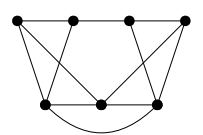

(h)

Figure 3.4: Graphs for Claim 3.3.5, part I.

(3) There are exactly four 3-vertices. 
(3.1) Assume there are exactly three 4 -vertices, say $v_{3}, v_{2}, v_{1}$. Let $H=G\left[\left\{v_{3}, v_{2}, v_{1}\right\}\right]$ be the graph induced by $v_{3}, v_{2}, v_{1}$.

(3.1.1) Assume $H$ has no edges. Then $G$ has just one realization $K_{3,4}$, see graph (a) in Figure 3.4, which has a $3-\mathrm{NZF}$.

(3.1.2) Assume $H$ has exactly one edge. Then $G$ has just one realization (b) shown in Figure 3.4, which has a 3-NZF as well.

(3.1.3) Assume $H$ has exactly two edges. Consider the graph $K$ induced by other vertices $v_{4}, v_{5}, v_{6}, v_{7}$. Then $K$ is either two 2-paths or one 3 -path together with an isolate vertex, see (c) and (d) in Figure 3.4. Define a function $\ell: V(G) \rightarrow \mathbb{Z}$ with $\ell\left(v_{4}\right)=\ell\left(v_{6}\right)=3, \ell\left(v_{7}\right)=\ell\left(v_{5}\right)=-3, \ell\left(v_{1}\right)=\ell\left(v_{2}\right)=\ell\left(v_{3}\right)=0$. It is routine to justify that $\left|\partial_{G}(A)\right| \geq\left|\sum_{u \in A} \ell(u)\right|, \forall A \subset V(G)$. By Lemma 3.3.3, G admits an orientation $D$ with $\ell(s)=d_{G}^{+}(s)-d_{G}^{-}(s)$, for any $s \in V(G)$. So this gives a modulo 3-orientation of $G$, thus a 3 -NZF in each case.

(3.1.4) Assume $H$ has exactly 3 edges. Then $G$ has 4 realizations (e), (f) (g) and (h) as in Figure 3.4. Thus we easily get that each of the graphs (g) and (h) has a 3-NZF, while the graphs (e) and (f) not.

(3.2) Assume there are exactly two 5-vertices and one 4-vertex. Suppose that there are two adjacent 3 -vertices, and say that $v_{2} \sim v_{1}$ by symmetry. Since $\left|E\left(G^{\prime}\right)\right|=(2 \cdot 5+4 \cdot 3+$ $4) / 2=13$, one has that $v_{7}, v_{6}, v_{5}, v_{4}, v_{3}$ induce a graph with 8 edges, which is (b) in Figure 3.3 or $W_{4}$. But $W_{4} \in\left\langle S \mathbb{Z}_{3}\right\rangle$ by Lemma 3.3.4, then we have $G^{\prime}$ does not contain $W_{4}$ as it is $\left\langle S \mathbb{Z}_{3}\right\rangle$-reduced. Thus we obtain that $v_{3}, v_{4}, v_{5}, v_{6}, v_{7}$ precisely induce the graph (b) in Figure 3.3. Notice also that $v_{3}, v_{4}, v_{5}, v_{6}$ induce a $K_{4}$, which is also an odd wheel $W_{3}$. Denote two partitions of $\left\{v_{6}, v_{5}, v_{4}, v_{3}\right\}$ by $\mathcal{P}_{1}=\left\{v_{5}, v_{3}\right\} \cup\left\{v_{6}, v_{4}\right\}$ and $\mathcal{P}_{2}=\left\{v_{4}, v_{5}\right\} \cup\left\{v_{3}, v_{6}\right\}$, respectively. By Lemma 3.3.5, with a careful analysis we can choose an appropriate partition from $\mathcal{P}_{i}$ for some $i \in\{1,2\}$, say $\mathcal{P}_{i}=A \cup B$, such that $G_{[A, B]}^{\prime}$ admits a $3-\mathrm{NZF}$. Hence $G^{\prime}$ has a $3-\mathrm{NZF}$, a contradiction. This shows that there is no adjacent 3 -vertices. Hence $G^{\prime} \cong K_{3,4}^{+}$.

(4) There are five 3 -vertices. Then there is a 5 -vertex, say $v_{1}$, and a 4 -vertex, say $v_{2}$.

(4.1) Assume $v_{1}$ is not adjacent to $v_{2}$. Then $v_{2}$ and $v_{1}$ have 4 common neighbor vertices. Hence $G^{\prime}$ is isomorphic to the graph (a) in Figure 3.5, which does not have a 3-NZF.

(4.2) Assume $v_{1}$ is adjacent to $v_{2}$, and that $v_{2}$ and $v_{1}$ have exactly 3 common neighbor vertices. Then the graph must be (b) in Figure 3.5. It is straightforward to check that it has a $3-\mathrm{NZF}$.

(4.3) Assume $v_{1}$ is adjacent to $v_{2}$, and that $v_{2}$ and $v_{1}$ have exactly 2 common neighbor 


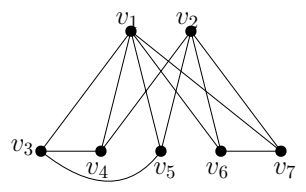

(a)

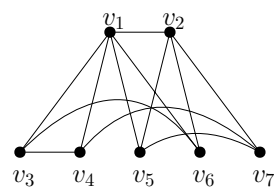

(d)

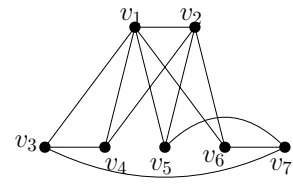

(b)

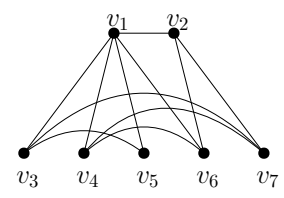

(e)

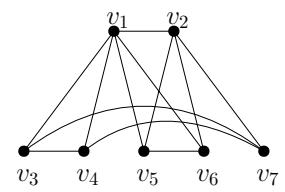

(c)

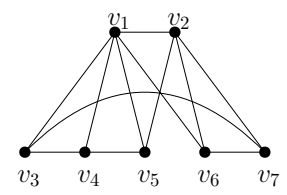

(f)

Figure 3.5: Graphs for Claim 3.3.5, part II.

vertices. Then there are four such graphs, (c), (d), (e) or (f) in Figure 3.5. We can check one by one that the graphs (c) and (e) do not have 3-NZF.

(5) There are six 3-vertices. Then $G^{\prime}$ has exactly one 4 -vertex. By Ore condition, there exist two 3 -vertices, say $v_{2}$ and $v_{1}$, such that $v_{2} \nsim v_{1}$. We have 3 subcases dividing by the number of common neighbor vertices of $v_{1}$ and $v_{2}$.

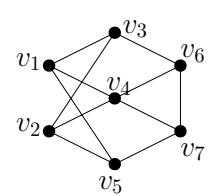

(a)

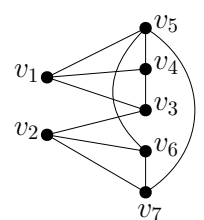

(f)

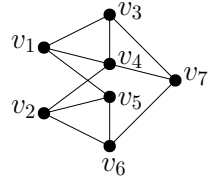

(b)

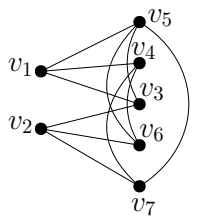

(g)

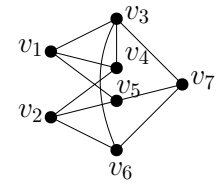

(c)

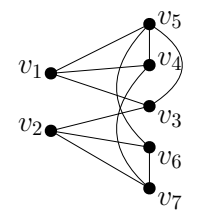

(h)

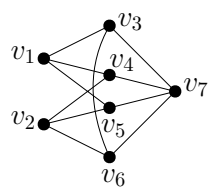

(d)

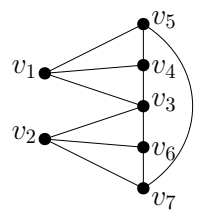

(i)

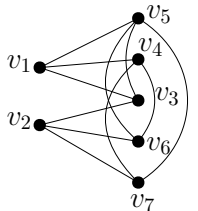

(e)

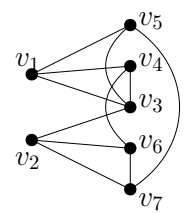

(j)

Figure 3.6: Graphs for Claim 3.3.5, part III.

(5.1) Assume $v_{1}$ and $v_{2}$ have 3 common neighbor vertices, say $v_{5}, v_{4}, v_{3}$. If $v_{7}$ or $v_{6}$ is a 4 -vertex, then such a graph does not exist. So assume instead that one of $v_{5}, v_{4}, v_{3}$ is a 4 -vertex, say $v_{3}$. If $v_{3} \sim v_{4}$, then there is no graph satisfied above condition. Hence, $G^{\prime}$ must be the graph (a) in Figure 3.6, which does not have a 3-NZF.

(5.2) Assume $v_{1}$ and $v_{2}$ have exactly 2 common neighbor vertices, say $v_{3}, v_{4}$. Assume the other neighbor vertex of $v_{1}$ and of $v_{2}$ is $v_{5}$ and $v_{6}$, respectively. If $v_{4}$ or $v_{5}$ is a 4 -vertex, then $G^{\prime}$ is (b) in Figure 3.6, which does not admit a $3-\mathrm{NZF}$. If $v_{3}$ or $v_{6}$ is a 4 -vertex, 
then $G^{\prime}$ is (c) in Figure 3.6, which admits a 3-NZF. If $v_{7}$ is the 4 -vertex, then $G^{\prime}$ is (d) in Figure 3.6, which does not admit a $3-\mathrm{NZF}$.

(5.3) Assume $v_{1}$ and $v_{2}$ have exactly one common neighbor vertex, say $v_{3}$. Assume the extra two neighbor vertices of $v_{1}$ and $v_{2}$ are $\left\{v_{4}, v_{5}\right\}$ and $\left\{v_{6}, v_{7}\right\}$, respectively. If one of $v_{7}, v_{6}, v_{5}, v_{4}$ is a 4-vertex, without loss of any generality, say $v_{5}$, then $G^{\prime}$ is one of the graphs (e), (f), (g) or (h) in Figure 3.6. Hence they all do not have a 3-NZF with an easy one by one verification. Now assume $v_{3}$ is the 4 -vertex. Then $G^{\prime}$ is one of the graphs (i) and (j) in Figure 3.6, which does not have a 3-NZF in each case.

By Claims 3.3.1-3.3.5, we conclude that if $G^{\prime} \notin \mathcal{M}_{3}$, then $G^{\prime} \in \mathcal{G}_{1} \cup K_{4} \cup\left\{K_{3, t}^{+}: t \geq 3\right\}$ as desired. 


\section{Chapter 4}

\section{Weighted Modulo Orientations of Graphs}

This chapter includes joint work with Ping Li, Jiaao Li and Hong-Jian Lai, appeared in [56].

\subsection{Introduction}

The current study is motivated by Theorems 1.2.7, 1.2.8 and 1.2.9. We are going to investigate the relationship between the edge-connectivity of a graph embedded on a 2-manifold and its $(f, b ; p)$-orientability over the finite field $\mathbb{Z}_{p}$. We follow $[28]$ to define a 2-cell (or cellular) embedding of a graph $G$ into a closed surface $S$ to be a continuous one-to-one function $i: G \rightarrow S$ if every component of $S-i(G)$ is homeomorphic to an open disk. In this paper, all embeddings of graphs are assumed to be 2-cell. We use $g$ to denote the Euler genus of $G$, which is the minimal integer $k$ such that the graph can be embedding into an orientable surface of genus $k / 2$ or into a nonorientable surface of genus $k$. Our main result is the following.

Theorem 4.1.1. Let $p>0$ be an odd prime, and let $G$ be a graph with Euler genus $g$ and edge connectivity

$$
\kappa^{\prime}(G) \geq \begin{cases}4 p-6+\lfloor g / 2\rfloor & \text { if } g \leq 2, \\ (p-2)\lfloor\sqrt{6 g+0.25}+2.5\rfloor+1 & \text { if } g \geq 3 \\ p \sqrt{4.98 g} & \text { if } g \text { is sufficiently large. }\end{cases}
$$

Then for any mapping $f \in F\left(G, \mathbb{Z}_{p}^{*}\right)$ and any $\mathbb{Z}_{p}$-boundary $b$ of $G$, the graph $G$ has an $(f, b ; p)$ orientation.

The next section will be focused on developing the needed mechanisms to derive our main result, utilizing additive bases in the linear space of the boundaries of a given graph, and 
contractible configurations of the related properties. The proof of the main result will be in the last section.

\subsection{Preliminaries}

Throughout this section, $\mathcal{F}, n$ and $p$ denote a field, a positive integer and an odd prime, respectively. We use $\mathcal{F}^{n}$ to denote the $n$-dimensional vector space over $\mathcal{F}$. For a graph $G$ on $n>0$ vertices, let $Z\left(G, \mathbb{Z}_{k}\right)$ denote the collection of all $\mathbb{Z}_{k}$-boundaries of $G$. By definition, $Z\left(G, \mathbb{Z}_{p}\right)$ is isomorphic to $\mathbb{Z}_{p}^{n-1}$.

\subsubsection{Additive bases of $Z\left(G, \mathbb{Z}_{p}\right)$}

Given a subset $S \subseteq \mathbb{Z}_{p}$, an $S$-additive basis of $\mathbb{Z}_{p}^{n}$ is a multiset $\left\{x_{1}, x_{2}, \cdots, x_{m}\right\} \subseteq \mathbb{Z}_{p}^{n}$ such that for any $x \in \mathbb{Z}_{p}^{n}$, there exist scalars $c_{i} \in S$ such that $x=\sum_{i=1}^{m} c_{i} x_{i}$, which is called an $S$-linear-combination of $x$. An additive basis is a $\{0,1\}$-additive basis. As indicated in [36], the $\bmod p$-orientation problem of graphs is closely related to the existence of additive bases of vector spaces over $\mathbb{Z}_{p}$, the field on $p$ elements.

Let $B_{1}, \ldots, B_{t}$ be a collection of bases of $\mathcal{F}^{n}$. Define $\uplus_{i=1}^{t} B_{i}$ to be the (multiset) union with repetitions of $B_{1}, \ldots, B_{t}$. Let $c(n, \mathcal{F})$ be the smallest positive integer $t$ such that for any $t$ bases $B_{1}, \ldots, B_{t}$ of $\mathcal{F}^{n}$, the multiset $\uplus_{i=1}^{t} B_{i}$ is an additive basis of $\mathcal{F}^{n}$. Define $c(n, p)=c\left(n, \mathbb{Z}_{p}\right)$. In the following, Theorem 4.2.1 (i) can be derived from Cauchy-Davenport Theorem in [17] (see Theorem 4.2.4), and Theorem 4.2.1 (ii) verified a former conjecture by H. B. Mann and J. E. Olson.

Theorem 4.2.1. Each of the following holds.

(i) (Davenport [17], see also [5]) If $p \geq 3$ is a prime, then $c(1, p)=p-1$.

(ii) (Mann and Wou [63]) If $p \geq 3$ is a prime, then $c(2, p)=p-1$.

We develop some more lemmas for our arguments deployed in this research.

Lemma 4.2.2. Let $x, y \in \mathcal{F}$ distinct elements. Then each of the following holds.

(i) If $A=\left\{a_{1}, \ldots, a_{m}\right\}$ is an $\{x, y\}$-additive basis of $\mathcal{F}^{n}$, then $(y-x) A=\left\{(y-x) a_{1}, \ldots,(y-\right.$ $\left.x) a_{m}\right\}$ is an additive basis of $\mathcal{F}^{n}$.

(ii) If $A=\left\{a_{1}, \ldots, a_{m}\right\}$ is an additive basis of $\mathcal{F}^{n}$, then $(y-x)^{-1} A=\left\{(y-x)^{-1} a_{1}, \ldots,(y-\right.$ $\left.x)^{-1} a_{m}\right\}$ is an $\{x, y\}$-additive basis of $\mathcal{F}^{n}$.

Proof. Let $\beta$ be an arbitrary vector in $\mathcal{F}^{n}$.

(i) Then $\beta+\sum_{i=1}^{m} x a_{i} \in \mathcal{F}^{n}$. As $\left\{a_{1}, \ldots, a_{m}\right\}$ is an $\{x, y\}$-additive basis of $\mathcal{F}^{n}$, there exist scalars $c_{1}, \ldots, c_{m} \in\{x, y\}$ such that $\beta+\sum_{i=1}^{m} x a_{i}=\sum_{i=1}^{m} c_{i} a_{i}$. For each $i \in\{1,2, \ldots, m\}$, let $d_{i}=(y-x)^{-1}\left(c_{i}-x\right)$. Thus if $c_{i}=x$ then $d_{i}=0$, and if $c_{i}=y$ then $d_{i}=1$. It follows that 
$\beta=(y-x)(y-x)^{-1} \sum_{i=1}^{m}\left(c_{i}-x\right) a_{i}=\sum_{i=1}^{m} d_{i}(y-x) a_{i}$ with $d_{i} \in\{0,1\}$, and so $(y-x) A$ is an additive basis of $\mathcal{F}^{n}$.

(ii) Then $\beta-(y-x)^{-1} \sum_{i=1}^{m} x a_{i} \in \mathcal{F}^{n}$. Since $\left\{a_{1}, \ldots, a_{m}\right\}$ is an additive basis of $\mathcal{F}^{n}$, there exist $c_{1}, \ldots, c_{m} \in\{0,1\}$ such that $\beta-(y-x)^{-1} \sum_{i=1}^{m} x a_{i}=\sum_{i=1}^{m} c_{i} a_{i}$. For each $i \in\{1,2, \ldots, m\}$, let $d_{i}=(y-x) c_{i}+x$. As $c_{i} \in\{0,1\}$, we have $d_{i} \in\{x, y\}$. It follows that $\beta=\sum_{i=1}^{m}\left((y-x) c_{i}+\right.$ $x)(y-x)^{-1} a_{i}=\sum_{i=1}^{m} d_{i}(y-x)^{-1} a_{i}$, and so $(y-x)^{-1} A$ is a $\{x, y\}$-additive basis of $\mathcal{F}^{n}$.

Let $G$ be a connected graph with $n=|V(G)| \geq 1$. For each $e \in E(G)$, define $x_{e} \in F\left(G, \mathbb{Z}_{p}\right)$ to be the characteristic function of $\{e\}$. Let $D$ be an arbitrary orientation of $G$. Recall that $Z\left(G, \mathbb{Z}_{p}\right)$ is isomorphic to $\mathbb{Z}_{p}^{n-1}$. Corollary 4.2 .3 reveals a relationship between additive bases in $Z\left(G, \mathbb{Z}_{p}\right)$ and the existence of an $(f, b ; p)$-orientation of $G$.

Corollary 4.2.3. Let $p \geq 3$ be a prime number, and let $G$ be a connected graph with $n=|V(G)|$. The following statements are equivalent.

(i) For any mapping $f \in F\left(G, \mathbb{Z}_{p}^{*}\right)$ and any $\mathbb{Z}_{p}$-boundary $b$ of $G, G$ has an $(f, b ; p)$-orientation.

(ii) For any given orientation $D_{1}$ of $G$ and for any mapping $f \in F\left(G, \mathbb{Z}_{p}^{*}\right)$, the multiset $\left\{f(e) \partial_{D_{1}}\left(x_{e}\right): e \in E(G)\right\}$ is a $\{-1,1\}$-additive basis of $Z\left(G, \mathbb{Z}_{p}\right)$.

(iii) For any given orientation $D_{2}$ of $G$ and for any mapping $f \in F\left(G, \mathbb{Z}_{p}^{*}\right)$, the multiset $\left\{2 f(e) \partial_{D_{2}}\left(x_{e}\right): e \in E(G)\right\}$ is an additive basis of $Z\left(G, \mathbb{Z}_{p}\right)$.

Proof. The equivalence between (ii) and (iii) is an immediate consequence of Lemma 4.2 .2 by letting $D_{1}=D_{2}$.

It remains to show that equivalence between (i) and (ii). Assume that (i) holds. For any mapping $f \in F\left(G, \mathbb{Z}_{p}^{*}\right)$ and any $b \in Z\left(G, \mathbb{Z}_{p}\right)$, by (i), $G$ admits an $(f, b ; p)$-orientation $D$. For each $e \in E(G)$, define $c_{e}=1$ if $e$ has the same orientation in both $D$ and $D_{1}$ and $c_{e}=-1$ if $e$ is oriented differently in $D$ and in $D_{1}$. By definition, we have $\partial_{D}(f)=b$, and so for each $v \in V(G)$,

$$
b(v)=\partial_{D}(f)(v)=\sum_{e \in E_{D}^{+}(v)} f(e)-\sum_{e \in E_{D}^{-}(v)} f(e)=\sum_{e \in E} c_{e} f(e) \partial_{D_{1}}\left(x_{e}\right)(v)
$$

Thus $b$ is a $\{1,-1\}$-linear-combination of vectors in $\left\{f(e) \partial_{D_{1}}\left(x_{e}\right): e \in E(G)\right\}$. By definition, the multiset $\left\{f(e) \partial_{D_{1}}\left(x_{e}\right): e \in E(G)\right\}$ is a $\{-1,1\}$-additive basis of $Z\left(G, \mathbb{Z}_{p}\right)$.

Conversely, we assume that the multiset $\left\{f(e) \partial_{D_{1}}\left(x_{e}\right): e \in E(G)\right\}$ is a $\{-1,1\}$-additive basis of $Z\left(G, \mathbb{Z}_{p}\right)$. For any $b \in Z\left(G, \mathbb{Z}_{p}\right)$, there exists scalars $c_{e} \in\{1,-1\}$ such that $b=$ $\sum_{e \in E(G)} c_{e} f(e) \partial_{D_{1}}\left(x_{e}\right)$. Let $D$ be an orientation obtained from $D_{1}$ such that for any edge $e \in E(G)$, $e$ has the same orientation in $D$ as in $D_{1}$ if $c_{e}=1$ and $e$ has an orientation in $D$ opposite to its orientation in $D_{1}$ if $c_{e}=-1$. It follows from $b=\sum_{e \in E(G)} c_{e} f(e) \partial_{D_{1}}\left(x_{e}\right)$ that $b=\partial_{D}(f)$, and so $D$ is an $(f, b ; p)$-orientation of $G$.

For a multisubset $\left\{x_{1}, \ldots, x_{k}\right\}$ of $\mathbb{Z}_{p}^{*}$, define $\Omega\left(x_{1}, \ldots, x_{k}\right)=\left\{\sum_{i=1}^{k} \ell_{i} x_{i}: \ell_{i} \in\{1,-1\}\right\}$ to be the set of $\{1,-1\}$-linear combinations of $\left\{x_{1}, \ldots, x_{k}\right\}$. By definition and since $p \geq 3$ is an odd 
prime,

$\Omega\left(x_{1}, \ldots, x_{k}\right)=-\Omega\left(x_{1}, \ldots, x_{k}\right)$, and so $\left|\Omega\left(x_{1}, \ldots, x_{k}\right)\right|$ is odd if and only if $0 \in \Omega\left(x_{1}, \ldots, x_{k}\right)$.

For two nonempty subsets $A, B \in \mathbb{Z}_{p}$, let $A+B=\{a+b: a \in A, b \in B\}$. The following result was proved by Cauchy [15] in 1813 and was later rediscovered by Davenport [17] in 1935.

Theorem 4.2.4. (Cauchy [15] and Davenport [17]) Let $p$ be a prime number, and $A$ and $B$ two nonempty subsets of $\mathbb{Z}_{p}$. Then $|A+B| \geq \min \{p,|A|+|B|-1\}$.

Lemma 4.2.5. Let $p$ be an odd prime and let $k$ be a positive integer with $1 \leq k<p$. If $\left\{x_{1}, \ldots, x_{k}\right\}$ is a multisubset of $\mathbb{Z}_{p}^{*}$, then $\left|\Omega\left(x_{1}, \ldots, x_{k}\right)\right| \geq k+1$.

Proof. We proceed by induction on $k$. If $k=1$, then $\Omega\left(x_{1}\right)=\left\{x_{1},-x_{1}\right\}$, and the lemma holds. Let $A=\Omega\left(x_{1}, \ldots, x_{k-1}\right)$. Then by induction, $\left|\Omega\left(x_{1}, \ldots, x_{k-1}\right)\right| \geq k$. Let $B=\left\{x_{k},-x_{k}\right\}$. Note that $\Omega\left(x_{1}, \ldots, x_{k}\right)=A+B$. By Theorem 4.2.4, $|A+B| \geq \min \{p,|A|+|B|-1\}=\min \{p, k+1\}=$ $k+1$, and so $\mid \Omega\left(x_{1}, \ldots, x_{k}\right) \geq k+1$.

\subsubsection{A family of graphs admitting $(f, b ; p)$-orientations}

For a graph $G$ and for each edge $u v \in E(G)$, let $[u v]$ denote the set of all (parallel) edges joining the two vertices $u$ and $v$. If $X \subseteq E(G)$ is an edge subset of a graph $G$, then the contraction $G / X$ is obtained from $G$ by identifying the two ends of each edge in $X$ and then deleting all the resulting loops. If $X=\{e\}$, we use $G / e$ for $G /\{e\}$. If $H$ is a connected subgraph of $G$, then we write $G / H$ for $G / E(H)$.

For a prime $p \geq 3$, let $\mathcal{O}_{p}$ denote the family of connected graphs such that a graph $G \in \mathcal{O}_{p}$ if and only if $G$ admits an $(f, b ; p)$-orientation for any $f \in F\left(G, \mathbb{Z}_{p}^{*}\right)$ and any $\mathbb{Z}_{p}$-boundary $b$. By definition, $K_{1} \in \mathcal{O}_{p}$. For a subgraph $H$ of a graph $G$, let $A_{G}(H)$ denote the vertices in $V(H)$ that are adjacent to some vertices in $V(G)-V(H)$ in $G$. (Vertices in $A_{G}(H)$ are called the vertices of attachment of $H$ in $G$.) We have the following proposition.

Proposition 4.2.1. Let $G$ be a connected graph. Then each of the following holds.

(i) If $G \in \mathcal{O}_{p}$ and $e \in E(G)$, then $G / e \in \mathcal{O}_{p}$.

(ii) If $H \subseteq G$ satisfying $H \in \mathcal{O}_{p}$ and $G / H \in \mathcal{O}_{p}$, then $G \in \mathcal{O}_{p}$.

Proof. (i) Let $e=\{u, v\}, G^{\prime}=G / e$ and $w$ be the vertex in $G^{\prime}$ onto which $e$ is contracted. Let $f^{\prime}: E\left(G^{\prime}\right) \rightarrow \mathbb{Z}_{p}^{*}$ and $b^{\prime}$ be an arbitrary $\mathbb{Z}_{p}$-boundary of $G^{\prime}$. Define mappings $f$ and $b$ as follows:

$$
f(h)=\left\{\begin{array}{ll}
f^{\prime}(h) & \text { if } h \in E\left(G^{\prime}\right)=E(G)-\{e\} \\
1 & \text { if } h=e .
\end{array} \text { and } b(z)= \begin{cases}b^{\prime}(z) & \text { if } z \in V(G)-\{u, v\} \\
b^{\prime}(w) & \text { if } z=u \\
0 & \text { if } z=v .\end{cases}\right.
$$




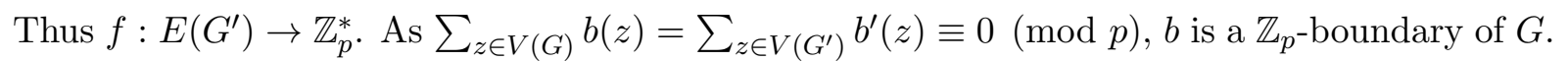
Since $G \in \mathcal{O}_{p}, G$ admits an $(f, b ; p)$-orientation $D$. Let $D^{\prime}$ be the restriction of $D$ to $E(G)-\{e\}$. Then $D^{\prime}$ can be viewed as an orientation of $G^{\prime}$. Since

$$
\begin{aligned}
\partial_{D^{\prime}} f^{\prime}(w) & =\sum_{e^{\prime} \in E_{D}^{+}(v) \cup E_{D}^{+}(u)-\{e\}} f\left(e^{\prime}\right)-\sum_{e^{\prime} \in E_{D}^{-}(v) \cup E_{D}^{-}(u)-\{e\}} f\left(e^{\prime}\right) \\
& =\partial_{D} f(u)+\partial_{D} f(v)=b(u)+b(v)=b^{\prime}(w),
\end{aligned}
$$

it follows that $\partial_{D^{\prime}} f^{\prime}=b^{\prime}$, and so $D^{\prime}$ is an $\left(f^{\prime}, b^{\prime} ; p\right)$-orientation of $G^{\prime}$. By definition, $G / e \in \mathcal{O}_{p}$. (ii) Suppose $H \in \mathcal{O}_{p}$ and $G / H \in \mathcal{O}_{p}$. By the definition of contraction, we may assume that $H$ is an induced subgraph of $G$, and so $E(G)$ is the disjoint union of $E(H)$ and $E(G / H)$. Let $v_{H}$ be the vertex in $G / H$ onto which $H$ is contracted. We verify the definition to show that $G \in \mathcal{O}_{p}$.

Arbitrarily take a $\mathbb{Z}_{p}$-boundary $b$ of $G$ and $f: E(G) \rightarrow \mathbb{Z}_{p}^{*}$. Let $a_{0}=\sum_{v \in V(H)} b(v)$. Define $b_{1}: V(G / H) \rightarrow \mathbb{Z}_{p}$ by

$$
b_{1}(z)= \begin{cases}b(z) & \text { if } z \in V(G / H)-\left\{v_{H}\right\} \\ a_{0} & \text { if } z=v_{H}\end{cases}
$$

As $b$ is a $\mathbb{Z}_{p}$-boundary, we have $\sum_{z \in V(G / H)} b_{1}(z)=\sum_{z \in V(G)} b(z)=0$, and so $b_{1}$ is a $\mathbb{Z}_{p}$-boundary of $G / H$. Let $f_{1}: E(G / H) \rightarrow \mathbb{Z}_{p}^{*}$ be the restriction of $f$ to $E(G / H)$. Since $G / H \in \mathcal{O}_{p}, G / H$ has an $\left(f_{1}, b_{1} ; p\right)$-orientation $D_{1}$. Define $b_{2}: V(H) \rightarrow \mathbb{Z}_{p}$ by

$$
b_{2}(z)= \begin{cases}b(z)+\sum_{e \in E_{D_{1}}^{-}\left(v_{H}\right) \cap E_{D}^{-}(z)} f_{1}(e)-\sum_{e \in E_{D_{1}}^{+}\left(v_{H}\right) \cap E_{D}^{+}(z)} f_{1}(e) & \text { if } z \in A_{G}(H) \\ b(z) & \text { otherwise. }\end{cases}
$$

As $a_{0}=\sum_{v \in V(H)} b(v)$, we have

$$
\sum_{z \in V(H)} b_{2}(z)=\sum_{z \in V(H)} b(z)+\sum_{e \in E_{D_{1}}^{-}\left(v_{H}\right)} f_{1}(e)-\sum_{e \in E_{D_{1}}^{+}\left(v_{H}\right)} f_{1}(e)=a_{0}-\partial_{D_{1}} f_{1}\left(v_{H}\right)=0,
$$

and so $b_{2}$ is a $\mathbb{Z}_{p}$-boundary of $H$. Let $f_{2}: E(H) \rightarrow \mathbb{Z}_{p}^{*}$ be the restriction of $f$ to $E(H)$. Since $H \in \mathcal{O}_{p}, H$ has an $\left(f_{2}, b_{2} ; p\right)$-orientation $D_{2}$. Obtain an orientation $D$ of $G$ by taking the union of $D_{1}$ and $D_{2}$. It remains to show that $D$ is an $(f, b ; p)$-orientation of $G$. For any vertex $z \in V(G)-A_{G}(H)$, by the definition of $D_{1}$ and $D_{2}$, we have $\partial_{D} f(z)=b(z)$. For any vertex $z \in A_{G}(H)$, by (4.5) and (4.6), it follows that

$$
\partial_{D} f(z)=\partial_{D_{1}} f_{1}(z)+\partial_{D_{2}} f_{2}(z)=\partial_{D_{1}} f_{1}(z)+b(z)-\partial_{D_{1}} f_{1}(z)=b(z) .
$$

Therefore $G \in \mathcal{O}_{p}$.

Nonempty families of connected graphs satisfying Proposition 4.2.1 (i) and (ii) are called complete families and investigated in $[13,14,43]$. Complete families have quite a few interesting properties and are associated with certain reduction methods. 
Corollary 4.2.6. Let $G$ be a connected graph and $p$ be an odd prime. Then $G \in \mathcal{O}_{p}$ if and only if every block of $G$ is in $\mathcal{O}_{p}$.

Proof. Let $B_{1}, B_{2}, \ldots, B_{c}$ be blocks of $G$. The corollary holds trivially if $c=1$, and so we assume $c \geq 2$. If $G \in \mathcal{O}_{p}$, then by Proposition 4.2.1 (i), $B_{i}=G /\left(\cup_{j \neq i} B_{j}\right) \in \mathcal{O}_{p}$. Conversely, assume that every $B_{i} \in \mathcal{O}_{p}$, we proceed by induction on $c$ to show that $G \in \mathcal{O}_{p}$. As $G / B_{c}$ has blocks $B_{1}, B_{2}, \ldots, B_{c-1}$ and $B_{i} \in \mathcal{O}_{p}$ for each $i \in\{1, \ldots, c-1\}$. By induction on $c$, we have that $G / B_{c} \in \mathcal{O}_{p}$. As $B_{c} \in \mathcal{O}_{p}$, by Proposition 4.2.1 (ii) we have that $G \in \mathcal{O}_{p}$.

For a given odd prime $p$, a graph $G$ is strongly $\mathbb{Z}_{p}$-connected if for any $f: E(G) \rightarrow$ $\{1,-1\} \subseteq \mathbb{Z}_{p}$, and any $\mathbb{Z}_{p}$-boundary $b, G$ admits an $(f, b ; p)$-orientation. The study of strongly $\mathbb{Z}_{p}$-connected graphs were initiated and investigated in $[42,44,46,48,49]$, among others. By definition, a graph is strongly $\mathbb{Z}_{3}$-connected if and only if it is $\mathbb{Z}_{3}$-connected. Lemma 4.2 .7 (i) follows from the definition, and Lemma 4.2 .7 (iv) follows from Lemma 4.2 .7 (i) and (iii).

Lemma 4.2.7. Let $p$ be an odd prime. Each of the following holds.

(i) Every graph $G \in \mathcal{O}_{p}$ is strongly $\mathbb{Z}_{p}$-connected.

(ii) (Jaeger et al., Proposition 2.2 of [36]) A graph $G$ is $\mathbb{Z}_{3}$-connected if and only if $G \in \mathcal{O}_{3}$.

(iii) (Proposition 3.9 of [49]) Every strongly $\mathbb{Z}_{p}$-connected graph contains $p-1$ edge-disjoint spanning trees.

(iv) Every graph in $\mathcal{O}_{p}$ contains $p-1$ edge-disjoint spanning trees and is thus $(p-1)$-edgeconnected.

For an integer $m>0$ and a graph $H$, define $H^{(m)}$ to be the graph obtained from $H$ by replacing each edge of $H$ by a set of $m$ parallel edges joining the same pair of vertices. In particular, $K_{2}^{(m)}$ is a loopless graph on two vertices and $m$ edges. Lemma 4.2.8 is a consequence of Theorem 4.2.1 (i), Corollary 4.2.3 and Lemma 4.2 .7 (iv).

Lemma 4.2.8. Let $G$ be a graph and $p$ be an odd prime. Then $K_{2}^{(m)} \in \mathcal{O}_{p}$ if and only if $m \geq p-1$.

Lemma 4.2.9. (Jaeger et al. [36]) A graph $G=(V, E)$ is connected if and only if for any $b \in Z\left(G, \mathbb{Z}_{p}\right)$ and for any orientation $D$, there exists and $f \in F\left(G, \mathbb{Z}_{p}\right)$ such that $\partial f=b$.

Let $|V(G)|=n$, and let the underlying simple graph of the graph $G$ be $C_{n}$, where $V(G)=$ $\left\{v_{j}: j \in \mathbb{Z}_{n}\right\}$. We denote $C_{n}$ the cycle with the same vertex set and such that $v_{j} v_{j+1}$ is an edge for each $j \in \mathbb{Z}_{n}$. Similarly, we denote $C_{n}\left(i_{1}, \ldots, i_{n}\right)$ the graph with the same vertex set and such that $i_{j}=\left|\left[v_{j} v_{j+1}\right]\right|$ for each $j \in \mathbb{Z}_{n}$. By definition, $C_{2}\left(i_{1}, i_{2}\right)=K_{2}^{\left(i_{1}+i_{2}\right)}$.

Lemma 4.2.10. Let $G=C_{n}\left(i_{1}, i_{2}, \ldots, i_{n}\right)$. If for each $j \in \mathbb{Z}_{n}, i_{j} \leq p-1$, and if $\sum_{j=1}^{n} i_{j} \geq$ $(n-1)(p-1)$, then $G \in \mathcal{O}_{p}$. 
Proof. Let $f \in F\left(G, \mathbb{Z}_{p}^{*}\right)$ and $b \in Z\left(G, \mathbb{Z}_{p}\right)$ be given. We are going to find an orientation $D$ of $G$ such that $\partial_{D}(f)=b$. Orient the edges of $E\left(C_{n}\right)$ so that for each $j \in \mathbb{Z}_{n}$, the edge $e_{j}$ is oriented from $v_{j}$ to $v_{j+1}$, and let $D_{1}$ denote the resulting orientation of $C_{n}$.

By Lemma 4.2.9, there is a mapping $f_{0}^{\prime} \in F\left(C_{n}, \mathbb{Z}_{p}\right)$ such that $\partial_{D_{1}} f_{0}^{\prime}=b$. For each constant $c \in\{1, \ldots, p-1\}$, let $f_{c}^{\prime}$ be the mapping given by $f_{c}^{\prime}(e)=f_{0}^{\prime}(e)+c$ for any $e \in E\left(C_{n}\right)$. It follows that $\partial_{D_{1}} f_{c}^{\prime}=\partial_{D_{1}} f_{0}^{\prime}=b$.

Fix an arbitrary $j \in \mathbb{Z}_{n}$, and let $\left[e_{j}\right]$ denote the edges parallel to $e_{j}$ in $G$. By assumption, we may denote $\left[e_{j}\right]=\left\{e_{j}^{1}, \ldots, e_{j}^{i_{j}}\right\}$ (with $e_{j}=e_{j}^{1}$ ). Define a bipartite graph $K$ with vertex bipartition $\left(V_{1}, V_{2}\right)$, where $V_{1}=\left\{f_{0}^{\prime}, f_{1}^{\prime}, \ldots, f_{p-1}^{\prime}\right\}$ and $V_{2}=\left\{e_{1}, e_{2}, \ldots, e_{n}\right\}$ such that $f_{c}^{\prime}$ is adjacent to $e_{j}$ in $K$ if and only if $f_{c}^{\prime}\left(e_{j}\right) \notin \Omega\left(f\left(e_{j}^{1}\right), \ldots, f\left(e_{j}^{i_{j}}\right)\right)$. Thus $d_{K}\left(e_{j}\right)=\left|\mathbb{Z}_{p}-\Omega\left(f\left(e_{j}^{1}\right), \ldots, f\left(e_{j}^{i_{j}}\right)\right)\right|$. By Lemma 4.2 .5 and since $i_{j} \leq p-1$ for each $j \in \mathbb{Z}_{n}$, we have $\sum_{j=1}^{n}\left|\Omega\left(f\left(e_{j}^{1}\right), \ldots, f\left(e_{j}^{i_{j}}\right)\right)\right| \geq$ $\sum_{j=1}^{n}\left(i_{j}+1\right)$. It follows by the assumption $\sum_{j=1}^{n} i_{j} \geq(n-1)(p-1)$ that

$$
\begin{aligned}
|E(K)| & =\sum_{j=1}^{n} d_{K}\left(e_{j}\right)=\sum_{j=1}^{n}\left|\mathbb{Z}_{p}-\Omega\left(f\left(e_{j}^{1}\right), \ldots, f\left(e_{j}^{i_{j}}\right)\right)\right| \\
& =\sum_{j=1}^{n}\left|\mathbb{Z}_{p}\right|-\sum_{j=1}^{n}\left|\Omega\left(f\left(e_{j}^{1}\right), \ldots, f\left(e_{j}^{i_{j}}\right)\right)\right| \\
& \leq n p-\sum_{j=1}^{n}\left(i_{j}+1\right) \leq n(p-1)-\sum_{j=1}^{n} i_{j} \leq p-1 .
\end{aligned}
$$

Hence there exists at least one $c \in \mathbb{Z}_{p}$ such that $f_{c}^{\prime}$ is of degree zero in $K$. This implies that for any $j \in \mathbb{Z}_{n}$, we always have $f_{c}^{\prime}\left(e_{j}\right) \in \Omega\left(f\left(e_{j}^{1}\right), \ldots, f\left(e_{j}^{i_{j}}\right)\right)$.

Consider a $c \in \mathbb{Z}_{p}$ such that $f_{c}^{\prime}$ is of degree zero in $K$. We now construct an orientation $D$ of $G$ so that $\partial_{D} f=b$ to complete the proof. For each $j \in \mathbb{Z}_{n}$, we orient the edges $\left\{e_{j}^{1}, \ldots, e_{j}^{i_{j}}\right\}$. Since $f_{c}^{\prime}\left(e_{j}\right) \in \Omega\left(f\left(e_{j}^{1}\right), \ldots, f\left(e_{j}^{i_{j}}\right)\right)$, by the definition of $\Omega\left(f\left(e_{j}^{1}\right), \ldots, f\left(e_{j}^{i_{j}}\right)\right)$, there exist scalars $\ell_{t} \in\{1,-1\} \subset \mathbb{Z}_{p}$ such that $f_{c}^{\prime}\left(e_{j}\right)=\sum_{t=1} i_{j} \ell_{t} f\left(e_{j}^{t}\right)$. For each $t$ with $1 \leq t \leq i_{j}$, orient $e_{j}^{t}$ from $v_{j}$ to $v_{j+1}$ if $\ell_{t}=1$ and from $v_{j+1}$ to $v_{j}$ if $\ell_{t}=-1$. Denote the resulting orientation of $G$ by $D$. By the definition of $D$, we have

$$
\sum_{e \in E_{D}^{+}\left(v_{j}\right) \cap\left[e_{j}\right]} f(e)-\sum_{e \in E_{D}^{-}\left(v_{j}\right) \cap\left[e_{j}\right]} f(e)=f_{c}^{\prime}(e) .
$$

This implies that $\partial_{D} f=\partial_{D_{1}} f_{0}^{\prime}=b$, and so $D$ is an $(f, b ; p)$-orientation of $G$. This proves the lemma.

Corollary 4.2.11. Let $G=C_{n}\left(i_{1}, i_{2}, \ldots, i_{n}\right)$. The following are equivalent.

(i) $G \in \mathcal{O}_{p}$.

(ii) G has $p-1$ edge-disjoint spanning trees. 
Proof. By Lemma 4.2.7 (iv), we have (i) implies (ii). We proceed by induction to prove that (ii) implies (i), and assume that $G$ has $p-1$ edge-disjoint spanning trees. If $n=2$, then (i) follows from Lemma 4.2.8. Assume that $n \geq 3$ and that (ii) implies (i) for smaller values of $n$. If $C_{n}$ has an edge, say $e_{n}=v_{n} v_{1}$ with $\left|\left[e_{n}\right]\right| \geq p-1$, then we induce on $G^{\prime}=G /\left[e_{n}\right]$. As $G^{\prime}=C_{n-1}\left(i_{1}, i_{2}, \ldots, i_{n-1}\right)$ and as $G^{\prime}$ also has $p-1$ edge-disjoint spanning trees, $G^{\prime} \in \mathcal{O}_{p}$. By Lemma 4.2.8 and Proposition 4.2.1, we have $G \in \mathcal{O}_{p}$. Therefore, we may assume that $|[e]| \leq p-2$ for any $e \in E\left(C_{n}\right)$. Since $G$ has $p-1$ edge-disjoint spanning trees, we have $\sum_{j=1}^{n} i_{j}=|E(G)| \geq(n-1)(p-1)$, and so by Lemma 4.2.10, $G \in \mathcal{O}_{p}$.

\subsection{Proof of Theorem 1.3.9}

We first make some remarks before proving Theorem 4.1.1. In the original version of this paper, for a graph with large Euler genus $g$, we proved edge connectivity bound 2gp, roughly, through a different method. A referee of this paper kindly shared his/her ideas to improve the bound from the fact that every simple graph with Euler genus $g$ is $O(\sqrt{g})$-degenerate, which eventually helps us to achieve the current bound $(p-2)\lfloor\sqrt{6 g+0.25}+2.5\rfloor+1$ for $g \geq 3$. Digging deeper on those arguments and ideas, with the help of Theorem 4.3.1 below, we are also able to get a better bound $p \sqrt{4.98 g}$ for a sufficiently large $g$. We would like to thank the referees for very helpful suggestions.

Theorem 4.3.1. (Delcourt and Postle [20]) For a sufficiently large integer n, every simple graph on $n$ vertices with minimum degree at least $0.8274 n$ can be edge-decomposed into triangles if each vertex has degree even and its number of edges is divisible by 3.

The following is a consequence of Theorem 4.3.1.

Lemma 4.3.2. For a sufficiently large integer $n$, every simple graph on $n$ vertices with minimum degree at least $0.8275 n$ can be edge-decomposed into triangles, plus at most $0.5 n+7$ single edges.

Proof. Let $G$ be a graph on $n$ vertices with minimum degree at least $0.8275 n$. Then $G$ has a Hamiltonian cycle $C$ by Dirac's Theorem. Let $T$ be the set of odd degree vertices in $G$. Clearly, $|T|$ is even, and so let $|T|=2 t$, where $t \geq 0$. We label the vertices of $T$ as $v_{1}, v_{2}, \ldots, v_{2 t}$ in the cyclic order along the Hamiltonian cycle $C$. Then for each $1 \leq i \leq t$, there is a path $P_{i}$ in the cyclic order of $C$ from $v_{2 i-1}$ to $v_{2 i}$. Define $X=\cup_{i=1}^{t} E\left(P_{i}\right)$ if $\left|\cup_{i=1}^{t} E\left(P_{i}\right)\right| \leq 0.5 n$, and $X=E(C) \backslash\left(\cup_{i=1}^{t} E\left(P_{i}\right)\right)$ otherwise. Then we have $|X| \leq 0.5 n$ and each vertex of $T$ has degree odd in $X$. Let $G_{1}=G-X$. Then each vertex of $G_{1}$ has degree even. If $\left|E\left(G_{1}\right)\right|$ is divisible by 3 , then let $G_{2}=G_{1}$. If $\left|E\left(G_{1}\right)\right|$ is not divisible by 3 , noting that $G_{1}$ contains both 5-cycles and 7 -cycles by Turán's Theorem, then we delete the edges of a 5 -cycle or a 7 -cycle in $G_{1}$ to obtain a new graph $G_{2}$ whose number of edges is divisible by 3 . Now $G_{2}$ has minimum degree at least 
$0.8275 n-4>0.8274 n$, and each vertex of $G_{2}$ has degree even. So Theorem 4.3.1 is applicable for $G_{2}$ in any case. Hence $E\left(G_{2}\right)$ can be edge-decomposed into triangles by Theorem 4.3.1. As $E(G) \backslash E\left(G_{2}\right)$ has at most $0.5 n+7$ edges, the lemma follows.

Now we are going to prove Theorem 4.1.1. As Theorem 4.1.1 holds trivially if $G=K_{1}$, we assume that $|V(G)| \geq 2$. In the following, we always let $\tilde{G}$ denote the underlying simple graph of $G$. For fixed integer $p \geq 3$, define a function on the interval $[3, \infty)$ as follows.

$$
\phi(x)=\frac{2(x-1)}{x-2} p-\frac{2 x}{x-2} .
$$

As on $[3, \infty)$, the derivative of the function is

$$
\phi^{\prime}(x)=\frac{4-2 p}{(x-2)^{2}}<0,
$$

it follows that

$$
\phi(x) \text { is a decreasing function on }[3, \infty) .
$$

We prove the following equivalent statement of Theorem 4.1.1.

Theorem 4.3.3. Let $p>0$ be an odd prime, and let $G$ be a graph with $\kappa^{\prime}(G) \geq p-1$. Then each of the following holds.

(i) If $G$ has Euler genus $g \leq 2$ and $\kappa^{\prime}(G) \geq 4 p-6+\lfloor g / 2\rfloor$, then $G \in \mathcal{O}_{p}$.

(ii) If $G$ has Euler genus $g \geq 3$ and $\kappa^{\prime}(G) \geq(p-2)\lfloor 2.5+\sqrt{6 g+0.25}\rfloor+1$, then $G \in \mathcal{O}_{p}$.

(iii) If $G$ has sufficiently large Euler genus (independent of $p$ ) and $\kappa^{\prime}(G) \geq p \sqrt{4.98 g}$, then $G \in \mathcal{O}_{p}$.

Proof. To prove Theorem 4.3.3, we argue by contradiction and assume that

$$
G \text { is a counterexample to Theorem } 4.3 .3 \text { with }|V(G)| \text { minimized. }
$$

Thus one of (i), (ii) and (iii) holds but $G \notin \mathcal{O}_{p}$, and so by (4.8), we have the following claim.

Claim 4.3.1. Each of the following holds.

(i) $\kappa(G) \geq 2$.

(ii) $G$ does not have a nontrivial subgraph $H$ such that $H \in \mathcal{O}_{p}$.

(iii) $G$ does not have a subgraph isomorphic to a $K_{2}^{(m)}$ with $m \geq p-1$.

(iv) $G$ does not have a subgraph isomorphic to a $C_{\ell}\left(i_{1}, i_{2}, \ldots, i_{\ell}\right)$ with $\sum_{j=1}^{\ell} i_{j} \geq(\ell-1)(p-1)$.

Since $\kappa^{\prime}(G) \geq p-1 \geq 2, G$ is connected. Let $B_{1}, B_{2}, \ldots, B_{c}$ be blocks of $G$. If $c \geq 2$, then the definition of edge-connectivity implies $\kappa^{\prime}(G)=\min \left\{\kappa^{\prime}\left(B_{i}\right): 1 \leq i \leq c\right\}$, and so by (4.8), each $B_{i} \in \mathcal{O}_{p}$. It follows by Corollary 4.2.6 that $G \in \mathcal{O}_{p}$, a contradiction to (4.8). Thus, $c=1$ and Claim 4.3.1 (i) holds. 
Let $H$ be a subgraph of $G$ such that $|V(H)|>1$ and $H \in \mathcal{O}_{p}$. Let $G^{\prime}=G / H$ with Euler genus $g^{\prime}$. Then by definition, $\kappa^{\prime}\left(G^{\prime}\right) \geq \kappa^{\prime}(G)$ and $g \geq g^{\prime}$. As $|V(H)|>1,\left|V\left(G^{\prime}\right)\right|<|V(G)|$, and so by (4.8), $G^{\prime} \in \mathcal{O}_{p}$. By Lemma 4.2.1 (ii), we have $G \in \mathcal{O}_{p}$, a contradiction to (4.8). Thus Claim 4.3.1 (ii) holds.

By Lemma $4.2 .8, K_{2}^{(m)} \in \mathcal{O}_{p}$ when $m \geq p-1$, and by Lemma $4.2 .10, C_{\ell}\left(i_{1}, i_{2}, \ldots, i_{\ell}\right) \in \mathcal{O}_{p}$ when $\sum_{j=1}^{\ell} i_{j} \geq(\ell-1)(p-1)$. Hence Claim 4.3 .1 (iii) and (iv) are consequences of Claim 4.3.1 (ii), and so the claim holds.

Notice that if $n=|V(G)| \leq 3$, then by Claim 4.3.1 (i), we have that the underling simple graph $\tilde{G}$ is isomorphic to $K_{n}$. When $n=2,3$, the edge connectivity implies that $G$ contains a subgraph in $\mathcal{O}_{p}$ (as in Claim 4.3.1 (iii) or (iv)), contrary to Claim 4.3.1 (ii). Hence we have

Observation 4.3.1. $|V(G)| \geq 4$.

By Claim 4.3.1 (iii), for any edge $e \in E(G)$, there are at most $p-2$ edges parallel to $e$ in $G$; and if $G$ has a subgraph $J$ isomorphic to a $C_{\ell}\left(i_{1}, i_{2}, \ldots, i_{\ell}\right)$, then $|E(J)| \leq(\ell-1)(p-1)-1$. This is a key fact in later proofs.

Let $S$ be a surface of Euler genus $g$ and suppose $G$ is embedded into $S$ in such a way that for each edge $e \in E(G)$, if $[e]=\left\{e^{1}, e^{2}, \ldots, e^{s}\right\}$ with $s=|[e]| \geq 2$, then, re-embedding the edges in $[e]$ if needed, the 2-cycles $\left\{e^{1}, e^{2}\right\},\left\{e^{2}, e^{3}\right\}, \ldots,\left\{e^{s-1}, e^{s}\right\}$ are the boundaries of some 2-faces of the embedding.

Define $F(G)$ to be the set of faces of $G$. For each $f \in F(G)$, we define $d_{G}(f)$ to be the number of edges incident with $f$, and for each integer $i \geq 1$, let $F_{i}$ be the number of faces of degree $i$ in $G$. A face of degree $\ell$ is often called an $\ell$-face. If the two edges of a 2 -face are parallel to or contain an edge of an $\ell$-face for some $\ell \geq 3$, then we say this 2 -face is related to the $\ell$-face, or is a related 2 -face of the $\ell$-face.

Recall Euler's formula that

$$
|V(G)|+|F(G)|-|E(G)|=2-g .
$$

To find a contradiction, we use a discharging argument. Define $k$ as follows,

$$
k= \begin{cases}4 p-6+\lfloor g / 2\rfloor & \text { if } g \leq 2, \\ (p-2)\lfloor\sqrt{6 g+0.25}+2.5\rfloor+1 & \text { if } g \geq 3 \\ p \sqrt{4.98 g} & \text { if } g \text { is sufficiently large }\end{cases}
$$

As in a 2-cell embedding of a graph $G$ on a surface, every edge is incident with one or two faces. It follows that every 2 -face of $G$ in this 2-cell embedding is related to either one or two faces of degree at least 3 . Define, for $i \in\{1,2\}$,

$X_{i}(G)=\{f \in F(G): f$ is a 2 -face and is related to $i$ faces of degree at least 3 in the embedding. $\}$ 
For each face $f \in F(G)$, we assign an initial charge $w(f)$ equaling the degree of $f$ in the embedding. Now we define the discharging rule as follows.

For $\ell \geq 3$ and $i \in 1,2$, every $\ell$-face $f$ gives $\frac{2(3-i)}{k-2}$ to each of the 2 -faces in $X_{i}(G)$ related to $f$.

For any $f \in F(G)$, let $w^{*}(f)$ be the resulting charge of $f$ after recharging. As every 2-face in $F(G)$ is either in $X_{1}(G)$ or in $X_{2}(G)$, by the discharging rule, we conclude that

$$
\text { For any } 2 \text {-face } f \text { of } G, w^{*}(f)=2+\frac{4}{k-2} \text {. }
$$

For an integer $\ell \geq 3$ and for any $f \in F(G)$ with $d_{G}(f)=\ell$, let $\bar{E}(f)$ be the set of edges that are in 2-faces related to $f$ or contained in $f$, and let $E_{1}(f)$ be the set of edges in 2-faces related to $f$ and in $X_{1}(G)$. Let $Y$ be the edge-induced graph by $\bar{E}(f)-E_{1}(f)$ and assume that $Y$ has $c$ components. Note that each component of $\bar{E}(f)-E_{1}(f)$ is a $C_{\ell_{j}}\left(i_{1}^{j}, \ldots, i_{\ell_{j}}^{j}\right)$ for $j \in\{1,2, \ldots, c\}$. Here $C_{\ell_{j}}\left(i_{1}^{j}, \ldots, i_{\ell_{j}}^{j}\right)$ is a single vertex when $\ell_{j}=0$. We may, without loss of generality, assume all those single vertices are $C_{\ell_{j}}\left(i_{1}^{j}, \ldots, i_{\ell_{j}}^{j}\right)$ 's for $j \geq c^{\prime}+1$, where $c^{\prime} \leq c$. Hence $\ell=\sum_{j=1}^{c} \ell_{j}+2(c-1)=\sum_{j=1}^{c^{\prime}} \ell_{j}+2 c-2$, and so $\sum_{j=1}^{c^{\prime}} \ell_{j}=\ell+2-2 c$.

By Claim 3.4 (iii) and (iv), $|\bar{E}(f)| \leq(c-1)(p-2)+\sum_{j=1}^{c^{\prime}}\left(\left(\ell_{j}-1\right)(p-1)-1\right)$. By the discharging rule, for any $\ell$-face $f$ of $G$ with $\ell \geq 3$,

$$
\begin{aligned}
w^{*}(f) & \geq \ell-\frac{2}{k-2}\left[2(c-1)(p-3)+\sum_{j=1}^{c^{\prime}}\left(\left(\ell_{j}-1\right)(p-1)-1-\ell_{j}\right)\right] \\
& =\ell-\frac{2}{k-2}\left[2(c-1)(p-3)+(p-2) \sum_{j=1}^{c^{\prime}} \ell_{j}-p c^{\prime}\right] \\
& =\ell-\frac{2}{k-2}\left[2(c-1)(p-3)+(p-2)(\ell+2-2 c)-p c^{\prime}\right] \\
& =\ell-\frac{2}{k-2}\left[-p c^{\prime}-2 c+\ell(p-2)+2\right] .
\end{aligned}
$$

By the definition of 2-cell embedding and 2-connectivity of $G$, one has $c \geq c^{\prime} \geq 1$. Hence, for any $\ell$-face $f$ of $G$ with $\ell \geq 3$,

$$
w^{*}(f) \geq \ell-\frac{2}{k-2}[-p-2+\ell(p-2)+2]=\ell-(\ell p-2 \ell-p) \frac{2}{k-2} .
$$

By (4.1), we have that $\kappa^{\prime}(G) \geq k$. Then $2|E(G)| \geq \kappa^{\prime}(G)|V(G)| \geq k|V(G)|$. It follows from Euler's formula $|V(G)|+|F(G)|-|E(G)|=2-g$ that $\frac{k}{k-2}(|F(G)|-2+g) \geq|E(G)|$, and so $\sum_{i \geq 2}\left(2+\frac{4}{k-2}\right) f_{i}-\frac{2 k(2-g)}{k-2}=\frac{2 k}{k-2}(|F(G)|-2+g) \geq 2|E(G)|=\sum_{f \in F(G)} w(f)=\sum_{f \in F(G)} w^{*}(f)$.

Case A $g \in\{0,1,2\}$. 
Then $\kappa^{\prime}(G) \geq k=4 p-6+\lfloor g / 2\rfloor \geq 4 p-6$. Let $k^{\prime}=4 p-6$. By (4.7), for any $f \in F(G)$ with $d_{G}(f)=\ell \geq 3$, we have

$$
k \geq k^{\prime}=4 p-6=\phi(3) \geq \phi(\ell)=\frac{2(\ell-1)}{\ell-2} p-\frac{2 \ell}{\ell-2}=\frac{2 \ell p-2 p-2 \ell}{\ell-2},
$$

which is equivalent to $\left(k^{\prime}-2\right) \ell-2(\ell p-2 \ell-p) \geq 2 k^{\prime}$. Hence

$$
\ell-(\ell p-2 \ell-p) \frac{2}{k^{\prime}-2} \geq \frac{2 k^{\prime}}{k^{\prime}-2}
$$

If $g=0,1$, then $k^{\prime}=k$, and so by (4.11) and (4.14) we have for any $f \in F(G)$ with $d_{G}(f)=\ell \geq 3, w^{*}(f) \geq \frac{2 k}{k-2}=2+\frac{4}{k-2}$. This, together with (4.10), implies $\sum_{f \in F(G)} w(f)=$ $\sum_{f \in F(G)} w^{*}(f) \geq \sum_{i \geq 2}\left(2+\frac{4}{k-2}\right) f_{i}$, contrary to (4.12). Thus the theorem must hold in Case A with $g=0,1$.

Now assume that $g=2$. Then $k>k^{\prime}=4 p-6=\phi(3)$. It follows by (4.13) and by $k>k^{\prime}$ that (4.14) holds with strict inequality if we replace $k^{\prime}$ by $k$ in (4.14). This leads to $\ell-(\ell p-2 \ell-p) \frac{2}{k-2}>\frac{2 k}{k-2}$. This, together with (4.11), implies that for any $f \in F(G)$ with $d_{G}(f)=\ell \geq 3, w^{*}(f)>\frac{2 k}{k-2}=2+\frac{4}{k-2}$. Thus, in conjunction with (4.10), we have

$$
\sum_{f \in F(G)} w(f)=\sum_{f \in F(G)} w^{*}(f)>\sum_{i \geq 2}\left(2+\frac{4}{k-2}\right) f_{i},
$$

contrary to (4.12). This settles Case A.

In the rest of the arguments, we let $\delta=\delta(\tilde{G})$ to be the minimum degree of $\tilde{G}$, the underling simple graph of $G$. By Claim 4.3.1 (iii), for any edge $e \in E(G)$ there are at most $p-2$ edges parallel to edge $e$. Hence the minimum degree of $G$ is at most $(p-2) \delta$. This provides the following observation.

Observation 4.3.2. $(p-2) \delta \geq \kappa^{\prime}(G) \geq k$.

Case B $g \geq 3$.

In this case, by (4.9) and Observation 4.3.2, we have

$$
\delta(\tilde{G})=\delta \geq\lfloor\sqrt{6 g+0.25}+2.5\rfloor+\frac{1}{p-2}>\lfloor\sqrt{6 g+0.25}+2.5\rfloor .
$$

Note that $\delta$ is a positive integer. Thus we have

$$
\delta>\sqrt{6 g+0.25}+2.5
$$

Since $\tilde{G}$ is a simple graph, every face of the embedding of $\tilde{G}$ has degree at least 3 , and so $2|E(\tilde{G})|=\sum_{f \in F(\tilde{G})} d(f) \geq 3|F(\tilde{G})|$. Note that the Euler genus of $\tilde{G}$ is the same as the Euler 
genus of $G$. Applying Euler's formula $|V(\tilde{G})|+|F(\tilde{G})|-|E(\tilde{G})|=2-g$ for $\tilde{G}$, we have $\frac{2}{3}|E(\tilde{G})| \geq$ $|F(\tilde{G})|=2-g+|E(\tilde{G})|-|V(\tilde{G})|$, which gives

$$
g-2 \geq \frac{1}{3}|E(\tilde{G})|-|V(\tilde{G})|=\frac{1}{3}|V(\tilde{G})|\left(\frac{|E(\tilde{G})|}{|V(\tilde{G})|}-3\right) \geq \frac{1}{3}(\delta(\tilde{G})+1)\left(\frac{\delta(\tilde{G})}{2}-3\right) .
$$

Combining with (4.15), it follows that $g-2 \geq \frac{1}{6}\left(\delta^{2}-5 \delta-6\right)>\frac{1}{6}\left[(\sqrt{6 g+0.25}+2.5)^{2}-\right.$ $5(\sqrt{6 g+0.25}+2.5)-6]=g-2$, a contradiction. This settles Case B.

Case C $g$ is sufficiently large.

For any $\ell$-face $f$ of $G$ with $\ell \geq 3$, by (4.11), we have

$$
w^{*}(f) \geq \ell\left(1-\frac{2(p-2)}{k-2}\right)+\frac{2 p}{k-2} \geq 3\left(1-\frac{2(p-2)}{k-2}\right)+\frac{2 p}{k-2}=\frac{3 k-4 p+6}{k-2} .
$$

Thus, by (4.10) and (4.12), we have

$$
\sum_{i \geq 2}\left(2+\frac{4}{k-2}\right) f_{i}-\frac{2 k(2-g)}{k-2} \geq \sum_{f \in F(G)} w^{*}(f) \geq\left(2+\frac{4}{k-2}\right) f_{2}+\sum_{i \geq 3} \frac{3 k-4 p+6}{k-2} f_{i},
$$

which gives $\frac{2 k(g-2)}{k-2} \geq \frac{k-4 p+6}{k-2} \sum_{i \geq 3} f_{i}$ and

$$
2 k(g-2) \geq(k-4 p+6) \sum_{i \geq 3} f_{i} .
$$

Notice that, since $G$ is embedded into $S$, the embedding of $\tilde{G}$ on $S$ may be obtained from embedding $G$ by deleting parallel edges. So for any $\ell \geq 3$, each $\ell$-face of $G$ is exactly an $\ell$-face of $\tilde{G}$. Hence we have $\sum_{i \geq 3} f_{i}=|F(\tilde{G})|=2-g+|E(\tilde{G})|-|V(\tilde{G})|$. By (4.16), we have

$$
2 k(g-2) \geq(k-4 p+6)(2-g+|E(\tilde{G})|-|V(\tilde{G})|) .
$$

If $|V(\tilde{G})|>\frac{\delta}{0.828}+6$, then it follows from (4.17) and Obervation 4.3.2 that

$$
\begin{aligned}
2 k(g-2) & \geq(k-4 p+6)(2-g+|E(\tilde{G})|-|V(\tilde{G})|) \\
& \geq(k-4 p+6)\left(2-g+\frac{\delta}{2}|V(\tilde{G})|-|V(\tilde{G})|\right) \\
& \geq(k-4 p+6)\left(2-g+\left(\frac{\delta}{2}-1\right)\left(\frac{\delta}{0.828}+6\right)\right) \\
& \geq(k-4 p+6)\left(2-g+\left(\frac{k}{2(p-2)}-1\right)\left(\frac{k}{0.828(p-2)}+6\right)\right) .
\end{aligned}
$$

Since $k=p \sqrt{4.98 g}, \frac{k}{p-2}>\sqrt{4.98 g}$, and $g$ is sufficiently large, we further obtain from the above inequality that

$$
\begin{aligned}
2 p \sqrt{4.98 g}(g-2) & \geq(k-4 p+6)\left(2-g+\left(\frac{k}{2(p-2)}-1\right)\left(\frac{k}{0.828(p-2)}+6\right)\right) \\
& >(p \sqrt{4.98 g}-4 p+6)\left(2-g+(0.5 \sqrt{4.98 g}-1)\left(\frac{\sqrt{4.98 g}}{0.828}+6\right)\right) \\
& >(p \sqrt{4.98 g}-4 p+6)(2-g+3.007 g) \\
& >2.006 g p \sqrt{4.98 g}
\end{aligned}
$$


a contradiction.

Assume instead that $|V(\tilde{G})| \leq \frac{\delta}{0.828}+6<\frac{\delta}{0.8275}$. Then $\delta(\tilde{G})=\delta \geq 0.8275|V(\tilde{G})|$ and $|V(\tilde{G})| \geq \delta+1 \geq \sqrt{4.98 g}$ is sufficiently large. Hence Lemma 4.3 .2 is applicable to $\tilde{G}$. It follows by Lemma 4.3.2 that $\tilde{G}$ can be decomposed into edge-disjoint triangles, plus at most $0.5|V(G)|+7$ single edges. By Claim 4.3 .1 (iv), each such triangle of $\tilde{G}$ corresponds to at most $2 p-3$ edge of $G$, and each single edge corresponds to at most $p-2$ edge of $G$. As there are at most $\frac{1}{3} \cdot \frac{|V(G)|(|V(G)|-1)}{2}$ such triangles in $\tilde{G}$, this gives an estimation on the number of edges in $G$ as follows:

$$
|E(G)| \leq(2 p-3) \cdot \frac{|V(G)|(|V(G)|-1)}{6}+(p-2) \cdot(0.5|V(G)|+7)<\frac{2 p|V(G)|^{2}}{6} .
$$

Hence we have

$$
|V(\tilde{G})|=|V(G)|>\frac{6|E(G)|}{2 p|V(G)|} \geq \frac{3 k}{2 p}=\frac{3 p \sqrt{4.98 g}}{2 p}=1.5 \sqrt{4.98 g} .
$$

Thus, by (4.17) and since $\frac{\delta}{2} \geq \frac{k}{2(p-2)}>\frac{1}{2} \sqrt{4.98 g}+1$, we obtain a contradiction as follows:

$$
\begin{aligned}
2 p \sqrt{4.98 g}(g-2)=2 k(g-2) & \geq(k-4 p+6)\left(2-g+\frac{\delta}{2}|V(\tilde{G})|-|V(\tilde{G})|\right) \\
& >(k-4 p+6)\left(2-g+\left(\frac{\delta}{2}-1\right) \cdot 1.5 \sqrt{4.98 g}\right) \\
& >(p \sqrt{4.98 g}-4 p+6)(2-g+0.75 \cdot 4.98 g) \\
& >2.5 g p \sqrt{4.98 g},
\end{aligned}
$$

a contradiction. This completes the proof for this case and justifies Theorem 4.3.3. 


\section{Chapter 5}

\section{Weighted Modulo Orientations of Graphs and Signed Graphs}

This chapter includes joint work with Han and Lai, appeared in [57].

\section{$5.1 \quad$ Introduction}

The current study is motivated by Theorems 1.2.7, 1.2.8 and 1.2.9. We are to investigate the relationship between the edge-connectivity of graphs in certain graph families and the $(f, b ; p)$ orientability of these graphs over the finite field $\mathbb{Z}_{p}$. First, we prepare some of the tools for our arguments in the proofs. We then will show improved edge-connectivity bounds in certain graph families in Sections 2-3. Section 4 is devoted to the study of signed graph, in which we introduce the $(f, b ; p)$-orientation of signed graphs and show that every $\left(12 p^{2}-28 p+15\right)$-edge-connected signed graph admits a $(f, b ; p)$-orientation. Further discussions and conjectures are presented in the last section.

For an integer $k>0$, let $\mathbb{Z}_{k}$ denote the (additive) cyclic group of order $k$ with additive identity 0 , and let $\mathbb{Z}_{k}^{*}=\mathbb{Z}_{k}-\{0\}$. A $\mathbb{Z}_{k}$-boundary of a graph $G$ is a mapping $b: V(G) \rightarrow \mathbb{Z}_{k}$ satisfying $\sum_{v \in V(G)} b(v) \equiv 0(\bmod k)$. The set of all $\mathbb{Z}_{k}$-boundaries of $G$ is denoted by $Z\left(G, \mathbb{Z}_{k}\right)$. Let $A \subseteq \mathbb{Z}_{k}$, and define $F(G, A)=\{f: E(G) \rightarrow A\}$. Fix an orientation $D=D(G)$ for a graph $G$. For any $f \in F\left(G, \mathbb{Z}_{k}\right)$, define $\partial_{D}(f): V(G) \rightarrow \mathbb{Z}_{k}$ as

$$
\partial_{D}(f)(v)=\sum_{e \in E_{D}^{+}(v)} f(e)-\sum_{e \in E_{D}^{-}(v)} f(e) .
$$

When the orientation $D$ is understood from the context, we often omit the subscript $D$ in the notation above and write $\partial f$ for $\partial_{D}(f)$. A mapping $f \in F\left(G, \mathbb{Z}_{k}\right)$ if a $Z_{k}$-flow if $\partial f=0$. It is known that for any $f \in F\left(G, \mathbb{Z}_{k}\right), \partial f$ is always a $\mathbb{Z}_{k}$-boundary. Let $\mathbb{F}$ denote a finite field and let $p>1$ denote an odd prime number. It has been noted that the study of modulo orientation 
of graphs is closely related to additive bases over finite fields. The following theorem by Alon et at. [4] indicates the existence of $c(n, p)$, where the logarithm function is of base 2 .

Theorem 5.1.1. (Alon, Linial and Meshulam [4]) $c(n, p) \leq(p-1) \log n+p-2$.

Lemma 5.1.2. (Esperet, de Joannis de Verclos, Le and Thomassé, Lemma 9 of [23]) Let $k \geq 1$ be an integer $p=2 k+1$ be an odd prime, and let $D=(V, A)$ be a digraph. For every arc $e \in A(D)$, let $L(e)$ be a pair of distinct elements of $\mathbb{Z}_{2 k+1}$. For any mapping $f: E \rightarrow \mathbb{Z}_{2 k+1}-\{0\}$ and any $\mathbb{Z}_{2 k+1}$-boundary $b$, the underlying non-oriented graph has an $(f, b ; p)$-orientation if and only if $D$ has a $\mathbb{Z}_{2 k+1}$-flow $g$ satisfying $\partial g=b$ and $g(e) \in L(e)$, for any $e \in A(D)$.

The following lemma is a summary of some basic properties of the graphs admitting $(f, b ; p)$ orientations. The proofs are slight modifications of those in $[42,44]$ justifying the corresponding results for modulo orientations and strong group connectivity of graphs.

Lemma 5.1.3. ( $[56])$ Let $G$ be a graph. Then for any $f \in F\left(G, \mathbb{Z}_{p}^{*}\right)$ and any $b \in Z\left(G, \mathbb{Z}_{p}\right)$, each of the following holds:

(i) $K_{1} \in \mathcal{O}_{p}$.

(ii) If $G \in \mathcal{O}_{p}$ and $e \in E(G)$, then $G / e \in \mathcal{O}_{p}$.

(iii) If $H \subseteq G$ satisfying $H \in \mathcal{O}_{p}$ and $G / H \in \mathcal{O}_{p}$, then $G \in \mathcal{O}_{p}$.

(iv) $G \in \mathcal{O}_{p}$ if and only if every block of $G$ is in $\mathcal{O}_{p}$.

(v) Every graph in $\mathcal{O}_{p}$ contains $(p-1)$ edge-disjoint spanning trees.

(vi) $m K_{2} \in \mathcal{O}_{p}$ if and only if $m \geq p-1$.

Suppose that for given $f \in F\left(G, \mathbb{Z}_{p}^{*}\right)$ and $b \in Z\left(G, \mathbb{Z}_{p}\right), D$ is an $(f, b ; p)$-orientation. Let $e_{0}=u v \in E(G)$ such that $(u, v) \in A(D)$, and $f^{\prime} \in F\left(G, \mathbb{Z}_{p}^{*}\right)$ be a mapping satisfying $f^{\prime}(e)=f(e)$ if $e \neq e_{0}$, and $f^{\prime}\left(e_{0}\right)=-f\left(e_{0}\right)$ in $\mathbb{Z}_{p}$. Define $D^{\prime}$ to be the orientation of $G$ by reverse the orientation of $e_{0}$ from $(u, v)$ to $(v, u)$. Then by definition, $D^{\prime}$ is an $\left(f^{\prime}, b ; p\right)$-orientation of $G$. This leads to the following observation.

Observation 5.1.1. Let $p$ be an odd prime. If for any $f: E(G) \rightarrow\left\{1,2, \ldots, \frac{p-1}{2}\right\}$, and for any $b \in Z\left(G, \mathbb{Z}_{p}\right), G$ always has an $(f, b ; p)$-orientation, then $G \in \mathcal{O}_{p}$.

Definition 5.1.1. Let $H$ be a subgraph of $G$, and let $s>0$ be an integer. The $\mathcal{O}_{p}$-closure of $H$ in $G$, denoted by $\mathrm{cl}(H)$, is the maximal subgraph of $G$ that contains $H$ such that $V(c l(H))-V(H)$ can be ordered as a sequence $\left\{v_{1}, v_{2}, \cdots, v_{t}\right\}$ such that there are at least $p-1$ edges joining $v_{1}$ and vertices in $H$, and for each $i$ with $1 \leq i \leq t-1$, there are at least $p-1$ edges joining $v_{i+1}$ and $V(H) \cup\left\{v_{1}, v_{2}, \cdots, v_{i}\right\}$.

As a corollary of Lemma 5.1 .3 (iii) and (vi), we have the following.

$$
\text { If } H \in \mathcal{O}_{p} \text {, then } c l(H) \in \mathcal{O}_{p} \text {. }
$$


Lemma 5.1.4. Let $T$ be a connected spanning subgraph of $G$. If for each edge e $\in E(T), G$ has a subgraph $H_{e} \in \mathcal{O}_{p}$ with $e \in E\left(H_{e}\right)$, then $G \in \mathcal{O}_{p}$.

Proof. We argue by induction on $|V(G)|$. Since $K_{1} \in \mathcal{O}_{p}$, the lemma holds trivially if $|V(G)|=1$. Assume that $|V(G)|>1$ and pick an arbitrary edge $e^{\prime} \in E(T)$. Then $G$ has a subgraph $H^{\prime} \in \mathcal{O}_{p}$ such that $e^{\prime} \in E\left(H^{\prime}\right)$. Let $G^{\prime}=G / H^{\prime}$ and let $T^{\prime}=T /\left(E\left(H^{\prime}\right) \cap E(T)\right)$. Since $T$ is a connected spanning subgraph of $G, T^{\prime}$ is a connected spanning subgraph of $G^{\prime}$. For each $e$ in $E\left(T^{\prime}\right)$, $e$ is also in $E(T)$, and so by assumption, $G$ has a subgraph $H_{e} \in \mathcal{O}_{p}$ with $e \in E\left(H_{e}\right)$. By Lemma 5.1.3(ii), $H_{e}^{\prime}=H_{e} /\left(E\left(H_{e}\right) \cap E\left(H^{\prime}\right)\right) \in \mathcal{O}_{p}$ and $e \in H_{e}^{\prime}$. Therefore by induction $G^{\prime} \in \mathcal{O}_{p}$. As $H^{\prime} \in \mathcal{O}_{p}$ and $G^{\prime}=G / H^{\prime} \in \mathcal{O}_{p}$, it follows from Lemma 5.1.3(iii) that $G \in \mathcal{O}_{p}$.

\subsection{Weighted Modulo Orientations of Certain Graphs}

In this section, we first investigate the edge connectivity of complete graphs in $\mathcal{O}_{p}$ and then apply it to study chordal graphs. We also determine, in Section 3.3, the sharp edge connectivity of series-parallel graphs in $\mathcal{O}_{p}$.

\subsubsection{Complete Graphs}

The main result of this subsection is the following theorem.

Theorem 5.2.1. If $n \geq 2(p-1)(5+3 \log (p-1))$, then the complete graph $K_{n}$ belongs to $\mathcal{O}_{p}$.

To show Theorem 5.2.1, we start with a lemma.

Lemma 5.2.2. Let $G$ be a graph of order $n$ with $c(n, p)$ edge-disjoint spanning trees. Then $G \in \mathcal{O}_{p}$.

Proof. Given an arbitrary $f \in F\left(G, \mathbb{Z}_{p}^{*}\right)$ and any $\mathbb{Z}_{p}$-boundary $b$. Let $T_{1}, \ldots, T_{c(n, p)}$ be edgedisjoint spanning trees of $G$, and $H=G\left[\cup_{t=1}^{c(n, p)} E\left(T_{i}\right)\right]$ be the subgraph induced by the edge subset $\cup_{t=1}^{c(n, p)} E\left(T_{i}\right)$. As $T_{i}$ 's are spanning trees of $G, H$ is a spanning subgraph of $G$. We shall first show that $H \in \mathcal{O}_{p}$.

Choose $T \in\left\{T_{1}, T_{2}, \ldots, T_{c(n, p)}\right\}$ and assign to $H$ an arbitrary orientation $D=D(H)$. Thus every subgraph of $H$ is a subdigraph of $D$ under this given orientation, and each $e \in E(H)$ is now an arc in $A(D)$. Let $n=|V(H)|$ and denote $A(T)=\left\{e_{1}, \ldots, e_{n-1}\right\}$. For each $e \in A(T)$, set $L(e)=\left\{a_{e}, b_{e}\right\}$ for two distinct elements $a_{e}, b_{e} \in \mathbb{Z}_{p}$.

Define a mapping $f_{0}: E(T) \rightarrow \mathbb{Z}_{p}$ by $f_{0}(e)=a_{e}$ for any $e \in E(T)$, and $f_{0}\left(e^{\prime}\right)=0$ if $e^{\prime} \notin E(T)$. Let $\partial f_{0}=b_{0}$ and $b^{\prime}=b-b_{0}$. As $b$ and $b_{0}$ are $\mathbb{Z}_{p}$-boundaries, $b^{\prime}$ is also a $\mathbb{Z}_{p}$-boundary of $G$. For any $e=\left(v_{i}, v_{j}\right) \in A(T)$, set $L^{\prime}(a)=\left\{0, b_{e}-a_{e}\right\}$ and define $x_{e}=\left(x_{1}^{e}, x_{2}^{e}, \ldots, x_{n}^{e}\right) \in \mathbb{Z}_{p}^{n}$ 
with

$$
x_{t}^{e}= \begin{cases}b_{e}-a_{e} & \text { if } t=i, \\ a_{e}-b_{e} & \text { if } t=j, \\ 0 & \text { otherwise }\end{cases}
$$

As $T$ is a spanning tree, $B(T)=\left\{x_{e}: e \in A(T)\right\}$ is a base of $\mathbb{Z}_{p}^{n}$. For each $i$ with $1 \leq i \leq n$, let $B_{i}=B\left(T_{i}\right)$. Then by the definition of $c(n, p)$, the union $B_{1} \cup \cdots \cup B_{c(n, p)}$ forms an additive basis of $\mathbb{Z}_{p}^{n}$. Thus there exist scalars $\lambda_{e} \in\{0,1\}$, where $e \in E\left(T_{1} \cup \cdots \cup T_{c(n, p)}\right)$, such that $\sum \lambda_{e} x_{e}=b-b_{0}$. Define $g_{0}: E(H) \rightarrow \mathbb{Z}_{p}$ by

$$
g_{0}(e)= \begin{cases}0 & \text { if } \lambda_{e}=0 \\ b_{e}-a_{e} & \text { if } \lambda_{e}=1 .\end{cases}
$$

Hence $\partial g_{0}=b-b_{0}$. Define $g: E\left(T_{1} \cup \cdots \cup T_{c(n, p)}\right) \rightarrow \mathbb{Z}_{p}$ by

$$
g(e)= \begin{cases}a_{e} & \text { if } g_{0}(e)=0, \\ b_{e} & \text { if } g_{0}(e)=b_{e}-a_{e} .\end{cases}
$$

Hence $\partial g=\partial g_{0}+b_{0}=b$ and $g(e) \in\left\{a_{e}, b_{e}\right\}$ for each $e \in E\left(T_{1} \cup \cdots \cup T_{c(n, p)}\right)$. By Lemma 5.1.2, $H$ has an $(f, b ; p)$-orientation. As $f$ and $b$ are arbitrarily given, $H \in \mathcal{O}_{p}$. Since $H$ is spanning in $G$, it follows by Lemma 5.1.3(i) and (iii) that $G \in \mathcal{O}_{p}$.

Proof of Theorem 5.2.1. When $p=3$, a graph $G \in \mathcal{O}_{p}$ which is equivalent to $G$ is $\mathbb{Z}_{3^{-}}$ connected. It is known that $K_{n}$ is $\mathbb{Z}_{3}$-connected if $n \geq 5$ (see Proposition 3.6 of [41]), and so theorem holds for $p=3$. In the following we assume $p \geq 5$.

Let $\phi(p)=2+2 \log (p-1)-\sqrt{2 \log (2 p-2)}$. Then as $\phi(2)=2-\sqrt{2}>0$ and when $p \geq 5$, $\phi^{\prime}(p)>0$, it follows that $2+2 \log (p-1) \geq \sqrt{2 \log (2 p-2)}$, and so algebraic manipulation leads to $5+3 \log (p-1) \geq \log (p-1)+\sqrt{2 \log (2(p-1))}+3=\log (2(p-1))+\sqrt{2 \log (2(p-1))}+2$. Consequently,

$$
n \geq 2(p-1)(5+3 \log (p-1)) \geq 2(p-1)(\log (2(p-1))+\sqrt{2 \log (2(p-1))}+1)+2(p-1) .
$$

Set

$$
x=\frac{n-2(p-1)}{2(p-1)}, \text { and } y=x-\log (2(p-1)) .
$$

By (6.2),

$$
x=\frac{n-2(p-1)}{2(p-1)} \geq \log (2(p-1))+\sqrt{2 \log (2(p-1))}+1, \text { and } y \geq \sqrt{2 \log (2(p-1))}+1 .
$$

By (6.3), $(y-1)^{2} \geq 2 \log (2(p-1))$, and so $1+y+\frac{1}{2}(y-1)^{2} \geq \log (2(p-1))+y+1$. Let $\psi(y)=$ $2^{y}-\left(1+y+\frac{1}{2}(y-1)^{2}\right)$. When $y \geq 3$, we have $\psi(3)=2>0$ and $\psi^{\prime}(y)=2^{y} \ln (2)-y>0$. 
It follows that as long as $y \geq 3,2^{y} \geq 1+y+\frac{1}{2}(y-1)^{2}$. Since $p \geq 5$, it follows by (6.3) that $y \geq \sqrt{2 \log (2(p-1))}+1 \geq \sqrt{6}+1>3$, and so we substitute $y-1$ by $\sqrt{2 \log (2(p-1))}$ in the inequality $2^{y} \geq 1+y+\frac{1}{2}(y-1)^{2}$ to obtain $2^{y} \geq \log (2(p-1))+y+1$. Hence $y \geq$ $\log (\log (2(p-1))+y+1)$, and so, as $x=\log (2(p-1))+y, y \geq \log (\log (2(p-1))+y+1)=\log (1+x)$. This implies that $x=\log (2(p-1))+y \geq \log (2(p-1))+\log (1+x)=\log (2(p-1)(1+x))$. Since $n-2(p-1)=2(p-1) x$ and $n \geq 2(p-1)(5+3 \log (p-1))$, one has $x \geq \log (n)$, and so $n=2(p-1) x+2(p-1) \geq 2(p-1) \log n+2(p-1) \geq 2(p-1) \log n+2(p-2)$. By Theorem 5.1.1, $\frac{n}{2} \geq(p-1) \log n+(p-2) \geq c(n, p)$. As $K_{n}$ has $\frac{n}{2}$ edge-disjoint spanning trees, by Lemma 5.2 .2 , we conclude that if $n \geq 4(p-1)(1+\log (p-1))$, then $K_{n} \in \mathcal{O}_{p}$.

\subsubsection{Chordal Graphs}

A simple graph $G$ is chordal if every cycle of length greater than 3 possesses a chord. Equivalently speaking, a simple graph $G$ is chordal if every induced cycle of $G$ has length 3 . We need the following structure property of chordal graphs.

Lemma 5.2.3. (Lemma 2.1.2 of [38]) A simple graph $G$ is chordal if and only if every minimal vertex-cut induces a clique of $G$.

The rest of this subsection is to show the following theorem.

Theorem 5.2.4. Every simple chordal graph $G$ with $\kappa(G) \geq 2(p-1)(5+3 \log (p-1))-1$ is in $\mathcal{O}_{p}$.

Proof. Let $k \geq 2(p-1)(5+3 \log (p-1))-1$ be an integer and $G$ be a chordal graph with $\kappa(G) \geq k$. If $G$ is a complete graph, say $G \cong K_{n}$, then $n \geq \kappa(G)+1 \geq 2(p-1)(5+3 \log (p-1))$ and $G \in \mathcal{O}_{p}$ by Theorem 5.2.1. Thus we assume $G$ is not a clique.

Let $e=x y \in E(G)$ be an arbitrary edge. By Lemma 5.2.2, it suffices to prove that $e$ lies in a subgraph $H_{e}$ of $G$ with $H_{e} \in \mathcal{O}_{p}$. We shall show that in any case, a subgraph $H_{e} \in \mathcal{O}_{p}$ with $e \in E\left(H_{e}\right)$ can always be found.

In the fist case, we assume that either $N_{G}(x) \neq V(G) \backslash\{x\}$ or $N_{G}(y) \neq V(G) \backslash\{y\}$. Then by symmetry, we assume $N_{G}(x) \neq V(G) \backslash\{x\}$. So there exists a vertex $z \in V(G)-\left(N_{G}(x) \cup\{x\}\right)$. Since $\kappa(G) \geq k \geq 2$ and $G$ is not a clique, $N_{G}(x)$ contains a minimal vertex-cut $X$ of $G$ separating $x$ and $z$. By Lemma $5.2 .3, G[X]$ is a clique, and so $G[X \cup\{x\}] \cong K_{m_{x}}$ with $m_{x}=$ $|X|+1 \geq \kappa(G)+1 \geq 2(p-1)(5+3 \log (p-1))$. By Lemma 5.2.1, $G[X \cup\{x\}] \in \mathcal{O}_{p}$. If $y \in X$, then as $G[X \cup\{x\}] \in \mathcal{O}_{p}$, we are done with $H_{e}=G[X \cup\{x\}]$. Hence we assume that

$$
\text { for any minimal vertex cut } X \subseteq N(x), y \notin X \text {. }
$$

If there exists $t \in N_{G}(y) \cap\left(V(G)-\left(N_{G}(x) \cup\{x\}\right)\right)$ such that $y t \in E(G)$, then there is a minimal vertex cut of $N_{G}(x)$ containing $y$ which separates $x$ and $t$, contrary to (5.4). It follows that 
$N_{G}(y) \subseteq N(x) \cup\{x\}$. Since $z \in V(G)-\left(N_{G}(x) \cup\{x\}\right)$, we have $y z \notin E(G)$, and so $N_{G}(y)$ contains a minimal vertex cut separating $y$ and $z$.

Let $Y$ be an arbitrarily chosen minimal vertex cut in $N_{G}(y)$ separating $y$ and $z$. By Lemma 5.2 .3 and as $\kappa(G) \geq 2(p-1)(5+3 \log (p-1))-1, G[Y \cup y] \cong K_{m_{y}}$ with $m_{y}=|Y|+1 \geq \kappa(G)+1 \geq$ $2(p-1)(5+3 \log (p-1))$. By Lemma 5.2.1, $G[Y \cup\{y\}] \in \mathcal{O}_{p}$. We may further assume that $x \notin Y$, as otherwise we are done with $H_{e}=G[Y \cup\{y\}] \in \mathcal{O}_{p}$. Thus $x y \in E(G-Y)$ and so $x$ and $y$ are in the same component of $G-Y$, and so $Y \subseteq N(y) \subseteq N(x) \cup\{x\}$ and $x \notin Y$. It follows that $H_{e}=G[Y \cup\{x, y\}]$ is a complete graph with order $|Y|+2 \geq \kappa(G)+2 \geq 2(p-1)(5+3 \log (p-1))+1$. By Lemma 5.2.1, $H_{e} \in \mathcal{O}_{p}$, and so this justifies the first case.

Otherwise, we may assume that both $N(x)=V(G) \backslash\{x\}$ and $N(y)=V(G) \backslash\{y\}$. Since $G$ itself is not a complete graph, $G$ contains vertices $v, v^{\prime} \in V(G)-\{x, y\}$ such that $v v^{\prime} \notin E(G)$. Therefore, $N(v)$ contains a minimal vertex cut $X^{\prime}$ separating $v$ and $v^{\prime}$ in $G$. By Lemma 5.2.3 and as $\kappa(G) \geq 2(p-1)(5+3 \log (p-1))-1, G\left[X^{\prime} \cup\{v\}\right]$ is a complete graph of order at least $2(p-1)(5+3 \log (p-1))$, and so by Lemma 5.2.1, it is in $\mathcal{O}_{p}$. Let $H_{e}=G\left[X^{\prime} \cup\{v\}\right]$. Since $N(x)=V(G)-x$ and $N(y)=V(G)-y$, both $x$ and $y$ must be in $X^{\prime}$, and so $e=x y \in E\left(H_{e}\right)$. This completes the proof of the lemma.

\subsubsection{Series-parallel graphs}

For a graph $G$, if $K_{4}$ can not be obtained from $G$ by deleting vertices or edges and by contraction, then $G$ is called $K_{4}$-minor free. In this section, we will present a sharp lower bound of edgeconnectivity for a $K_{4}$-minor free graph to be in $\mathcal{O}_{p}$. The following is a theorem of Dirac [21].

Theorem 5.2.5. (Dirac [21]) If $G$ is a simple $K_{4}$-minor free graph, then $\delta(G) \leq 2$.

Corollary 5.2.6. Let $G$ be a $K_{4}$-minor free graph. If $\kappa^{\prime}(G) \geq 2 p-3$, then $G \in \mathcal{O}_{p}$.

Proof. Let $G$ be a $(2 p-3)$-edge-connected $K_{4}$-minor free graph, and let $G_{0}$ be the underlying simple graph of $G$ (see p. 47 of [8]). By Lemma 5.1.3(i), $K_{1} \in \mathcal{O}_{p}$. Hence we assume that $|V(G)|>1$ and let $G$ be a minimal counterexample with $|V(G)|$ minimized.

Since $G$ is $K_{4}$-minor free, we have $G_{0}$ is also $K_{4}$-minor free. By Theorem 5.2.5, there is a vertex $w \in V\left(G_{0}\right)$ with degree 1 or 2 . If $d_{G_{0}}(w)=1$, since $\kappa^{\prime}(G) \geq 2 p-3$, we have a subgraph $H \subseteq G$ such that $H \cong(2 p-3) K_{2}$. If $d_{G_{0}}(w)=2$, let $e_{1}$ and $e_{2}$ be two edges incident with $w$ in $G_{0}$. By $\kappa^{\prime}(G) \geq 2 p-3$, at least one of $e_{1}$ and $e_{2}$ must be contained in a subgraph $H \subseteq G$ with $H \cong(p-1) K_{2}$. In either case, by Lemma 5.1.3(vi), $H \in \mathcal{O}_{p}$. Since $G$ is $K_{4}$-minor free, we have $G / H$ is also $K_{4}$-minor free. By the property of contractions, we have $\kappa^{\prime}(G / H) \geq \kappa^{\prime}(G)$. By the minimality of $G$, we obtain $G / H \in \mathcal{O}_{p}$. Since $H \in \mathcal{O}_{p}$ and by Lemma 5.1.3(iii), $G \in \mathcal{O}_{p}$, and so the corollary is complete. 


\subsection{Complete Bipartite Graphs and Graphs with Small Match- ing Number}

In this section we will determine sufficient conditions for a complete bipartite graph to be in $\mathcal{O}_{p}$. From definition, a graph $G$ is $\mathbb{Z}_{3}$-connected if and only if it is in $\mathcal{O}_{3}$. Since Theorem 4.6 of [16] characterizes all complete bipartite graphs in $\mathcal{O}_{3}$. Throughout this section, we assume that $p \geq 5$ is an odd prime. Using the arguments similar to those justifying Theorem 3.2 of [45], the following lifting lemma can be routinely verified from the definition of graphs in $\mathcal{O}_{p}$.

Lemma 5.3.1 (Lifting). Let $G$ be a graph and $p>0$ be an odd prime. For every function $f \in F\left(G, \mathbb{Z}_{p}^{*}\right)$ and any $\mathbb{Z}_{p}$-boundary $b$ of $G$, let $v_{1} v_{2}, v_{1} v_{3}$ be two edges of $G$ with $f\left(v_{1} v_{2}\right)=$ $f\left(v_{1} v_{3}\right)$. Let $G_{\left[v_{1}, v_{2} v_{3}\right]}$ be the graph obtained from $G$ by deleting $v_{1} v_{2}, v_{1} v_{3}$ and adding a new edge $e=v_{2} v_{3}$, and $f^{\prime} \in F\left(G_{\left[v_{1}, v_{2} v_{3}\right]}, \mathbb{Z}_{p}^{*}\right)$ be formed from the restriction of $f$ to $E-\left\{v_{1} v_{2}, v_{1} v_{3}\right\}$ by adding $f^{\prime}\left(v_{2} v_{3}\right)=f\left(v_{1} v_{2}\right)$. If $G_{\left[v_{1}, v_{2} v_{3}\right]}$ has an $\left(f^{\prime}, b ; p\right)$-orientation, then $G$ has an $(f, b ; p)$ orientation.

Definition 5.3.1. Let $G$ be a graph, $f \in F\left(G, \mathbb{Z}_{p}^{*}\right)$ and any $\mathbb{Z}_{p}$-boundary $b$ of $G$. Fix two vertices $u_{1}, u_{2} \in V(G)$ with $\left|N_{G}\left(u_{1}\right) \cap N_{G}\left(u_{2}\right)\right| \geq p-1$, (say $W=\left\{v_{1}, \ldots, v_{p-1}\right\} \subseteq N_{G}\left(u_{1}\right) \cap$ $\left.N_{G}\left(u_{2}\right)\right)$, satisfying that $f\left(u_{1} v_{i}\right)=f\left(u_{2} v_{i}\right)$ for each $i \in\{1, \ldots, p-1\}$. We obtain a new graph $G_{u_{1}, u_{2}, W}^{L}$ from $G$ by lifting each edge pair in $\left\{u_{1} v_{1}, u_{2} v_{1}\right\}, \ldots,\left\{u_{1} v_{p-1}, u_{2} v_{p-1}\right\}$. For notational convenience, when $u_{1}, u_{2}$ and $W$ are understood from the context, we simply use $G^{L}$ for $G_{u_{1}, u_{2}, W}^{L}$, and we say that $G^{L}$ is obtained by performing the L-operation on $G$ at $\left\{u_{1}, u_{2}\right\}$. By definition, $G^{L}$ contains a subgraph $L_{u_{1}, u_{2}}$ with vertex set $\left\{u_{1}, u_{2}\right\}$ and with at least $(p-1)$ multiple edges between $u_{1}, u_{2}$.

By Lemmas 5.1.3(vi), $L_{u_{1}, u_{2}} \in \mathcal{O}_{p}$ and so by Lemma 5.3.1,

$$
\text { if } G^{L} / L_{u_{1}, u_{2}} \in \mathcal{O}_{p} \text {, then } G \in \mathcal{O}_{p} \text {. }
$$

In the rest of this section, we define

$$
\begin{aligned}
n_{1} & =\frac{1}{2}(p-1)(p-2)+1, \\
n_{3} & =\frac{1}{2} n_{1}\left(n_{1}-1\right), \\
n_{2} & =n_{3}(p-1) .
\end{aligned}
$$

Theorem 5.3.2. Let $G=K_{n_{1}, n_{2}}$ and $p>0$ be an odd prime. For every function $f \in F\left(G, \mathbb{Z}_{p}^{*}\right)$ and every $\mathbb{Z}_{p}$-boundary $b$ of $G, G$ has an $(f, b ; p)$-orientation. Consequently, $K_{n_{1}, n} \in \mathcal{O}_{p}$ for every $n \geq n_{2}$. 
Proof. Let $b \in Z\left(G, \mathbb{Z}_{p}\right)$ and $f \in F\left(G, \mathbb{Z}_{p}^{*}\right)$ be given. We show that $K_{n_{1}, n_{2}}$ has an $(f, b ; p)$ orientation. By Observation 5.1.1, we may assume that

$$
\text { for any } e \in E(G), f(e) \in\left\{1, \ldots, \frac{p-1}{2}\right\} \text {. }
$$

Let $(U, V)$ denote the bipartition of $G=K_{n_{1}, n_{2}}$ with $U=\left\{u_{1}, \ldots, u_{n_{1}}\right\}$ and $V=\left\{v_{1}, \ldots, v_{n_{2}}\right\}$, and let $K_{n_{1}}$ be the complete graph with $V\left(K_{n_{1}}\right)=U$ and $E\left(K_{n_{1}}\right)=\left\{e_{1}, \ldots, e_{n_{3}}\right\}$.

Claim 5.3.1. Construct a new bipartite graph $B=B(G)$ with vertices partition $\left(W_{1}, W_{2}\right)$, where $W_{1}=V$ and $W_{2}=E\left(K_{n_{1}}\right)$, such that $v_{j}$ is adjacent to $e_{i}=u_{i_{1}} u_{i_{2}}$ if and only if $f\left(v_{j} u_{i_{1}}\right)=f\left(v_{j} u_{i_{2}}\right)$. If $|U|=n_{1}>\frac{p-1}{2}$, then each of the following satisfies.

(i) For any $v_{j} \in V, d_{B}\left(v_{j}\right) \geq 2$.

(ii) There exists an $e_{i} \in W_{2}$ with $d_{B}\left(e_{i}\right) \geq p-1$.

For any $v_{j} \in V$, by (5.7) and as $|U|=n_{1}>\frac{p-1}{2}$, there exist distinct $u_{i_{1}}, u_{i_{2}} \in U$ such that $f\left(v_{j} u_{i_{1}}\right)=f\left(v_{j} u_{i_{2}}\right)$. Hence $d_{B}\left(v_{j}\right) \geq 2$. Counting the number of edges in $H$, we have

$$
\sum_{v \in W_{1}} d_{B}(v)=|E(B)|=\sum_{e \in W_{2}} d_{B}(e)
$$

By (5.6) and as $p \geq 4, n_{2}>n_{3}(p-2)+1$, which, together with (5.8), implies that there exists an $e_{i} \in W_{2}$ with $d_{B}\left(e_{i}\right) \geq p-1$. This justifies Claim 5.3.1.

Assume that $e_{i}=u_{i_{1}} u_{i_{2}}$ is the edge assured in Claim 5.3.1(ii), and $N_{B}\left(e_{i}\right)$ contains $Q_{1}=$ $\left\{v_{j_{1}}, \ldots, v_{j_{p-1}}\right\} \subseteq W_{1}$. By the definition of $B$,

$$
\text { for any } \ell \in\{1, \ldots, p-1\}, f\left(u_{i_{1}} v_{j_{\ell}}\right)=f\left(u_{i_{2}} v_{j_{\ell}}\right) \text {. }
$$

Let $G^{L}=G_{u_{i_{1}}, u_{i_{2}}, Q_{1}}^{L}$ and $L_{u_{i_{1}}, u_{i_{2}}}$ be the graphs arising in the process of performing Loperations to $G$, as defined in Definition 5.3.1. Define $G_{1}=G^{L} / L_{u_{i_{1}}, u_{i_{2}}}, v_{L_{1}}$ be the vertex in $G_{1}$ onto which $L_{u_{i_{1}}, u_{i_{2}}}$ is contracted. and $G_{1}^{\prime}=G_{1}-Q_{1}$. Then $G_{1}^{\prime}$ is again a complete bipartite graph with bipartition $\left(U_{1}, V_{1}\right)$ where $U_{1}=\left(U-\left\{u_{i_{1}}, u_{i_{2}}\right\}\right) \cup\left\{v_{L_{1}}\right\}$ and $V_{1}=V-Q_{1}$, where $\left|U_{1}\right|=n_{1}-1$ and $\left|V_{1}\right|>\frac{1}{2}\left(n_{1}-1\right)\left(n_{2}-1\right)(p-1)$. Assuming that for some $j \geq 1$, the bipartite graph $G_{j}^{\prime}=\left(V_{j}, U_{j}\right)$ is defined. Form the bipartite graph $B\left(G_{j}^{\prime}\right)$ as in Claim 5.3.1. By applying Claim 5.3.1 with $G_{j}^{\prime}$ replacing $G$, there exists a vertex $e_{j}=u_{j_{1}} u_{j_{2}} \in E\left(K_{\left|V_{j}\right|}\right)$ of degree at least $p-1$ in $B\left(G_{j}^{\prime}\right)$, then a subset $Q_{j+1} \subseteq N_{B\left(G_{j}^{\prime}\right)}\left(e_{j}\right) \subseteq V_{j}$ is identified with $\left|Q_{j+1}\right|=p-1$. Let $G_{j}^{L}=\left(G_{j}^{\prime}\right)_{u_{j_{1}}, u_{j_{2}}, Q_{j+1}}^{L}$ with $L_{j+1}=L_{u_{j_{1}}, u_{j_{2}}}$ be the graphs arising in the process of performing L-operations to $G_{j}^{\prime}$. Let $G_{j+1}=\left(G_{j}^{\prime}\right)^{L} / L_{j+1}$, and $G_{j+1}^{\prime}=G_{j+1}-Q_{j+1}$. With the same arguments, $G_{j+1}^{\prime}$ is also a bipartite graph with bipartition $\left(U_{j+1}, V_{j+1}\right)$. As $G$ is finite, this process must ends at $j=\ell$ for some integer $\ell>0$, and so no further L-operations can be performed in the way above on the bipartite graph $G_{\ell}^{\prime}$. Let $\left(U_{\ell}, V_{\ell}\right)$ be the bipartition of $G_{\ell}^{\prime}$. 
Claim 5.3.2. $\left|U_{\ell}\right| \leq \frac{p-1}{2}$.

Assume by contradiction, we have $\left|U_{\ell}\right| \geq \frac{p-1}{2}+1$. Denote the restriction of $f$ to $E\left(G_{\ell}^{\prime}\right)$ as $f^{\prime}: E\left(G_{\ell}^{\prime}\right) \rightarrow \mathbb{Z}_{p}^{*}$. By (5.7), for any $e \in E\left(G_{\ell}^{\prime}\right)$, we have $f^{\prime}(e) \in\left\{1, \ldots, \frac{p-1}{2}\right\}$. Construct the bipartite graph $B\left(G_{\ell}^{\prime}\right)$ as in Claim 5.3.1. As $\left|U_{\ell}\right| \geq \frac{p-1}{2}+1$, both Claim 5.3.1, there exists an $e \in E\left(K_{\left|V_{\ell}\right|}\right)$ with $d_{B\left(G_{\ell}^{\prime}\right)}(e) \geq p-1$. As shown in the paragraph above, it is possible to perform an additional L-operation on the two ends of $e$. This contradict to the fact that no further L-operations can be performed on $G_{\ell}^{\prime}$. This proves Claim 5.3.2.

By Definition 5.3.1, there exists a sequence of ordered pairs $\left(L_{1}, Q_{1}\right),\left(L_{2}, Q_{2}\right), \ldots,\left(L_{\ell}, Q_{\ell}\right)$ arising in the process of the L-operations to obtain $G_{\text {ell }}$, and satisfying both (S1) and (S2) below.

(S1) Let $U_{0}=U$. For $i=1,2, \ldots, \ell$, each $L_{i}$ is spanned by a $(p-1) K_{2}$, with $V\left(L_{i}\right)$ consisting of two vertices in $U_{i-1}$, formed by, for $i>1$, identifying the two vertices in $V\left(L_{i-1}\right)$ in $U_{i-2}$.

(S2) Let $Q_{0}=\emptyset$. For $i=1,2, \ldots, \ell,\left|Q_{i}\right|=p-1, Q_{i} \subseteq V-\left(\cup_{j<i} Q_{j}\right)$, and no edges joining vertices in $Q_{i}$ to the contraction image of $L_{i}$.

Let $G^{\prime}$ be the graph obtained from $G$ by recursively applying the L-operation at the two vertices of each $L_{i}$, and then contract the edges in $E\left(L_{i}\right)$, recursively for each $i=1,2, \ldots, \ell$. As all the contractions are taken with vertices in $U, G^{\prime}$ is a bipartite graph with bipartition $\left(V, U_{\ell}\right)$. Since $|U|=\frac{1}{2}\left(p^{2}-3 p+4\right)=\frac{1}{2}(p-1)(p-2)+1$, by (S1) and Claim 5.3.2, there must be a vertex $u^{\prime} \in U_{\ell}$ which is obtained by identifying at least $p-1$ vertices in $U$. Let $V^{\prime}=V-\left(\cup_{j=1}^{\ell} Q_{j}\right)$. By (S2) and (5.6), as $\ell<n_{3}$, we have $\left|V^{\prime}\right| \geq n_{2}-\ell(p-1) \geq p-1$. It follows that for every $v^{\prime} \in V^{\prime}$, there are at least $(p-1)$ parallel edges joining $u^{\prime}$ and $v^{\prime}$ in $G^{\prime}$. Let $J=\operatorname{cl}\left(\left\{u^{\prime}\right\}\right)$, the $\mathcal{O}_{p^{-}}$-closure of the single vertex $u^{\prime}$ in $G^{\prime}$. By Definition 5.1.1, $V^{\prime} \subseteq V(J)$. By (S2), any vertex in $V^{\prime}$ is adjacent to every vertex in $U_{\ell}$. Since $\left|V^{\prime}\right| \geq p-1$, it follows by Definition 5.1.1, that $U_{\ell} \subseteq V^{\prime}$. By (S2) again, every $v \in V$ is in at most one $Q_{j}$ 's, and so by $p \geq 5, d_{G^{\prime}}\left(v^{\prime}\right) \geq d_{G}(v)-2=n_{1}-2 \geq p-1$. It follows that $G^{\prime}=J$ and so by (5.1), $G^{\prime} \in \mathcal{O}_{p}$.

Let $G^{\prime \prime}$ be the graph obtained from $G$ by recursively performing the L-operation at the two vertices of each $L_{i}$, recursively for each $i=1,2, \ldots, \ell$. Then by Definition 5.3.1, $G^{\prime \prime}$ is a bipartite graph with bipartition $(U, V)$ as $G$ with $E\left(G^{\prime \prime}\right)-\cup_{j=1}^{\ell} E\left(L_{j}\right) \subset E(G)$. Fix $j$ with $1 \leq j \leq \ell$, for each edge $e_{j} \in E\left(L_{j}\right)$, by (5.9), there exists a pair of edges $e_{j}^{\prime}, e_{j}^{\prime \prime} \in E_{G}(v)$ for some $v \in V$ with $f\left(e_{j}^{\prime}\right)=f\left(e_{j}^{\prime \prime}\right)$ such that in the lifting process, $e_{j}^{\prime}$ and $e_{j}^{\prime \prime}$ become $e_{j}$ in $G^{\prime \prime}$. Define $f^{\prime}\left(e_{j}\right)=f\left(e_{j}^{\prime}\right)$, for each edge $e_{j} \in E\left(L_{j}\right)$, where $1 \leq j \leq \ell$.

Recall that $b \in Z\left(G, \mathbb{Z}_{p}\right)$ and $f \in F\left(G, \mathbb{Z}_{p}^{*}\right)$ are given with $f$ satisfying (5.7). Define $b^{\prime}=b \in$ 
$Z\left(G, \mathbb{Z}_{p}\right)$ and $f^{\prime}: E\left(G^{\prime \prime}\right) \rightarrow \mathbb{Z}_{p}^{*}$ as follows:

$$
f^{\prime}(e)= \begin{cases}f(e) & \text { if } e \in E(G)-\cup_{j=1}^{\ell} E\left(L_{j}\right), \\ f^{\prime}(e) & \text { if } e \in \cup_{j=1}^{\ell} E\left(L_{j}\right) .\end{cases}
$$

By Lemma 5.1.3(iii) and (vi), and since $G^{\prime} \in \mathcal{O}_{p}$, we conclude that $G^{\prime \prime} \in \mathcal{O}_{p}$. Hence $G^{\prime \prime}$ has an $\left(f^{\prime}, b ; p\right)$-orientation $D^{\prime}$. By repeated application of Lemma 5.3.1, we conclude that $G$ has an $(f, b ; p)$-orientation, as desired.

For positive integers $m$ and $n$, let $K_{m, n}$ be the complete bipartite graph with bipartition $U=\left\{u_{1}, \ldots, u_{m}\right\}$ and $V=\left\{v_{1}, \ldots, v_{n}\right\}$. For any subset $\left\{t_{1}, t_{2}, \ldots, t_{\ell}\right\}$ of $\mathbb{Z}_{m}$, where $t_{1} \leq$ $t_{2} \ldots \leq t_{\ell}$, let $K\left(t_{1}, t_{2}, \ldots, t_{\ell}\right)$ be the graph obtained from $K_{m, n}$ by identifying $u_{1}, \ldots, u_{t_{1}}$, identifying $u_{t_{i}+1}, \ldots, u_{t_{i+1}}$ for each $1 \leq i \leq \ell-1$ and identifying $u_{t_{\ell}+1}, \ldots, u_{n}$, respectively. Define

$$
\mathcal{K}^{*}(m, n)=\left\{K\left(t_{1}, t_{2}, \ldots, t_{\ell}\right):\left\{t_{1}, t_{2}, \ldots, t_{\ell}\right\} \subseteq \mathbb{Z}_{m}\right\} .
$$

Since identifying two nonadjacent vertices $u, v$ in a graph $G$ amounts to the operation $(G+$ $u v) / u v$. By Lemma 5.1.3(iii) and (ii), $G \in \mathcal{O}_{p}$ implies that $(G+u v) / u v \in \mathcal{O}_{p}$. Combining Theorem 5.3.2, leads to the following seemingly more general corollary.

Corollary 5.3.3. Let $G \in \mathcal{K}^{*}\left(n_{1}, n_{2}\right)$ be a graph and $p>0$ be an odd prime. Then $G \in \mathcal{O}_{p}$.

As an application of corollary above, we present that if a family of graphs has a bounded matching number, then after certain reduction operations, there are only finitely many $\frac{1}{2}\left(p^{2}-\right.$ $3 p+4)$-edge-connected graphs not in $\mathcal{O}_{p}$. To state our theorem formally, we shall first introduce the concept of $\mathcal{O}_{p}$-reduction below.

As $K_{1} \in \mathcal{O}_{p}$ by definition, for every graph $G$, any vertex is contained in a maximal subgraph in $\mathcal{O}_{p}$. Let $H_{1}, H_{2}, \cdots, H_{c}$ be the family of all maximal subgraphs $G$ which all in $\mathcal{O}_{p}$. Define $G^{\prime}=G /\left(\cup_{i=1}^{c} E\left(H_{i}\right)\right)$ to be the $\mathcal{O}_{p}$-reduction of $G$, or $G$ is $\mathcal{O}_{p}$-reduced to $G^{\prime}$. If $G$ does not have any nontrivial subgraph in $\mathcal{O}_{p}$, then $G$ is called $\mathcal{O}_{p}$-reduced. Our main result can be stated below.

Theorem 5.3.4. Let $G$ be a graph, $p>0$ be an odd prime and $s>0$ be an integer. Then for every function $f \in F\left(G, \mathbb{Z}_{p}^{*}\right)$ and every $\mathbb{Z}_{p}$-boundary $b$ of $G$, there is a finite graph family $\mathcal{G}(p, s)$ such that every graph $G$ with $\kappa^{\prime}(G) \geq \frac{1}{2}\left(p^{2}-3 p+4\right)$ and $\alpha^{\prime}(G) \leq s$ has an $(f, b ; p)$-orientation if and only if the $\mathcal{O}_{p}$-reduction of $G$ is not in $\mathcal{G}(p, s)$.

To obtain this theorem, we also need the following elementary counting lemma, see [26, II.5*].

Lemma 5.3.5. ( [26]) Let $\ell, n>0$ be integers. Then there are $\left(\begin{array}{c}n+\ell-1 \\ \ell-1\end{array}\right)$ non-negative integral solutions $\left(x_{1}, x_{2}, \ldots, x_{\ell}\right)$ for the equation $x_{1}+x_{2}+\cdots+x_{\ell}=n$. 
Denote $N(p, s)=n_{2} \cdot\left(\begin{array}{c}2 s+n_{1}-1 \\ 2 s-1\end{array}\right)+2 s$, where $n_{1}=\frac{1}{2}\left(p^{2}-3 p+4\right), n_{2}=\frac{1}{2} n_{1}\left(n_{1}-1\right)(p-1)$. Let $\mathcal{F}(p, s)$ be the family of all $n_{1}$-edge-connected $\mathcal{O}_{p}$-reduced graphs of order between 2 and $N(p, s)$ with matching number at most $s$. Then each graph in $\mathcal{F}(p, s)$ has edge multiplicity at most $p-2$ by Lemma 5.1 .3 (vi). So there are finitely many graphs in $\mathcal{F}(p, s)$. We will show the following stronger theorem, which implies Theorem 5.3.4 by Lemma 5.1.3(1), (3) and Theorem 5.3.4.

Theorem 5.3.6. Let $G$ be a $\frac{1}{2}\left(p^{2}-3 p+4\right)$-edge-connected graph with $\alpha^{\prime}(G) \leq s$. Then $G \in \mathcal{O}_{p}$ if and only if $G$ cannot be $\mathcal{O}_{p}$-reduced to a member in $\mathcal{F}(p, s)$.

Proof. If $G \in \mathcal{O}_{p}$, then $G$ is $\mathcal{O}_{p}$-reduced to $K_{1} \notin \mathcal{F}(p, s)$ by Lemma 5.1.3(vi). We shall show the converse that if $G$ cannot be $\mathcal{O}_{p}$-reduced to a member in $\mathcal{F}(p, s)$, then $G \in \mathcal{O}_{p}$.

Let $G$ be a counterexample and let $G^{\prime}$ be the $\mathcal{O}_{p}$-reduction of $G$. Then $G^{\prime} \notin \mathcal{F}(p, s)$ and it leads to

$$
\left|V\left(G^{\prime}\right)\right|>N(p, s)=n_{2} \cdot\left(\begin{array}{c}
2 s+n_{1}-1 \\
2 s-1
\end{array}\right)+2 s .
$$

By the definition of $G^{\prime}$, we achieve $\alpha^{\prime}\left(G^{\prime}\right) \leq \alpha^{\prime}(G) \leq s$. Let $M=\left\{w_{1} w_{2}, w_{3} w_{4}, \ldots\right.$, $\left.w_{2 d-1} w_{2 d}\right\}$ be a maximum matching of $G^{\prime}$, where $d \leq s$. Denote $W=\left\{w_{1}, \ldots, w_{2 d}\right\}$. Then $Z=V\left(G^{\prime}\right)-W$ is an independent vertex set of $G^{\prime}$. Since $G^{\prime}$ is $n_{1}$-edge-connected, we have $\left|[z, W]_{G^{\prime}}\right| \geq n_{1}$ for any $z \in Z$. Pick arbitrary $n_{1}$ edges from $[z, W]_{G^{\prime}}$, denoted by $H(z)$, for each $z \in Z$. Let $G_{1}^{\prime}=\cup_{z \in Z} H(z)$ be the graph induced by the edge set $\cup_{z \in Z} H(z)$ in $G^{\prime}$.

We claim that there exists a member of $\mathcal{K}^{*}\left(n_{1}, n_{2}\right)$ in $G_{1}^{\prime}$, therefore in $G^{\prime}$. This will lead to a contradiction to the fact that $G^{\prime}$ is a $\mathcal{O}_{p}$-reduced graph by Theorem 5.3.3.

For any $w \in W$ and $z \in Z$, denote $x(w, z)=\left|[w, z]_{G_{1}^{\prime}}\right|$ to be the number of edges between $w$ and $z$ in $H(z)$. Note that $x(w, z)=0$ if $w$ is not in the graph $H(z)$. Since $H(z)$ consists of $n_{1}$ edges, we have, for each $z \in Z$,

$$
x\left(w_{1}, z\right)+x\left(w_{2}, z\right)+\cdots+x\left(w_{2 d}, z\right)=n_{1} .
$$

By (5.10) and $d \leq s,|Z|=\left|V\left(G^{\prime}\right)\right|-2 d>N(p, s)-2 s \geq n_{2}\left(\begin{array}{c}2 s+n_{1}-1 \\ 2 s-1\end{array}\right)$. By Lemma 5.3.5 and the Pigeon-Hole Principle, there exists a subset $Z_{1} \subset Z$ of size $n_{2}$ such that, for any $z, z^{\prime} \in Z_{1}$,

$$
\left(x\left(w_{1}, z\right), x\left(w_{2}, z\right), \ldots, x\left(w_{2 d}, z\right)\right)=\left(x\left(w_{1}, z^{\prime}\right), x\left(w_{2}, z^{\prime}\right), \ldots, x\left(w_{2 d}, z^{\prime}\right)\right) .
$$

Denote $x_{1}, \ldots, x_{\ell+1}$ to be all the nonzero coordinates in $\left(x\left(w_{1}, z\right), x\left(w_{2}, z\right), \ldots, x\left(w_{2 d}, z\right)\right)$. Then the graph $\left[S_{1}, Y\right]_{G_{1}^{\prime}} \cong K\left(t_{1}, t_{2}, \ldots, t_{\ell}\right)$ is a member of $\mathcal{K}^{*}\left(n_{1}, n_{2}\right)$, where $t_{1}=x_{1}, t_{\ell+1}=\left(n_{1}\right)-t_{\ell}$ and $t_{i}-t_{i-1}=x_{i}$ for $2 \leq i \leq \ell$. This proves the claim as well as the theorem.

\subsection{Signed graphs}

A signed graph is an ordered pair $(G, \sigma)$ consisting of a graph $G$ with a mapping $\sigma: E(G) \rightarrow$ $\{1,-1\}$. An edge $e \in E(G)$ is positive if $\sigma(e)=1$ and negative if $\sigma(e)=-1$. The mapping $\sigma$, 
called the signature of $G$, is sometimes implicit in the notation of a signed graph and will be specified when needed. Both negative and positive loops are allowed in signed graphs. Define $E^{+}(G)=\sigma^{-1}(1)$ and $E^{-}(G)=\sigma^{-1}(-1)$. If no confusion occurs, we simply use $E^{+}$for $E^{+}(G)$ and $E^{-}$for $E^{-}(G)$. An orientation $\tau$ assigns each edge of $(G, \sigma)$ as follows: if $e=x y \in E^{+}(G)$, then $e$ is either oriented from $x$ and to $y$ or from $y$ and to $x$; if $e=x y \in E^{-}(G)$, then $e$ is oriented either away from both $x$ and $y$ or towards both $x$ and $y$. We call $e=x y$ a sink edge (a source edge, respectively) if it is oriented away from (towards, respectively) both $x$ and $y$.

Let $\tau$ be an orientation of $(G, \sigma)$. For each vertex $v \in V(G)$, let $H_{G}(v)$ be the set of half edges incident with $v$. Define $\tau(h)=1$ if the half edge $h \in H_{G}(v)$ is oriented away from $v$, and $\tau(h)=-1$ if the half edge $h \in H_{G}(v)$ is oriented towards $v$. Denote $d_{\tau}^{+}(v)=\left|E^{+}(v)\right|\left(d_{\tau}^{-}(v)=\right.$ $\left|E^{-}(v)\right|$, respectively) to be the outdegree (indegree, respectively) of $(G, \sigma)$ under orientation $\tau$, where $E_{\tau}^{+}(v)\left(E_{\tau}^{-}(v)\right.$, respectively) denotes the set of outgoing (ingoing, respectively) half edges incident with $v$.

An edge cut of $(G, \sigma)$ is just an edge cut of $G$. The switch operation $\zeta=\zeta_{S}$ on an edge-cut $S$ is a mapping $\zeta: E(G) \rightarrow\{-1,1\}$ such that $\zeta(e)=-1$ if $e \in S$ and $\zeta(e)=1$ otherwise. Two signatures $\sigma$ and $\sigma^{\prime}$ are equivalent if there exists an edge-cut $S$ such that $\sigma(e)=\sigma^{\prime}(e) \zeta(e)$ for every edge $e \in E(G)$, where $\zeta$ is the switch operation on some edge-cut $S$ of $G$. For a signed graph $(G, \sigma)$, let $\chi$ denote the collection of all signatures equivalent to $\sigma$. The negativeness of $(G, \sigma)$ is denoted by $\epsilon_{N}(G, \sigma)=\min \left\{\left|E_{\sigma^{\prime}}^{-}(G)\right|: \forall \sigma^{\prime} \in \chi\right\}$. We use $\epsilon_{N}$ for short if the signed graph $(G, \sigma)$ is understood from the context. A signed graph is called $k$-unbalanced if $\epsilon_{N} \geq k$, and a 1-unbalanced signed graph is also known as an unbalanced signed graph.

We follow [50], to define signed graph contractions. For an edge $e \in E(G)$, the contraction $G / e$ is the signed graph obtained from $G$ by identifying the two ends of $e$, and then deleting the resulting positive loop if $e \in E^{+}$, but keeping the resulting negative loop if $e \in E^{-}$. For $X \subseteq E(G)$, the contraction $G / X$ is the signed graph obtained from $G$ by contracting each edge in $X$. If $H$ is a subgraph of $G$, then we use $G / H$ for $G / E(H)$. By definition, for any edge subset $X$ of $G, \epsilon_{N}(G / X) \leq \epsilon_{N}(G)$.

Let $A$ be an abelian (additive) group. Define $2 A=\{2 \alpha: \forall \alpha \in A\}$, and $A^{*}=A-\{0\}$. For a signed graph $(G, \sigma)$, we still denote $F(G, A)=\{f \mid f: E(G) \rightarrow A\}$. Let $\tau$ be an orientation of $(G, \sigma)$. For each $f \in F\left(G, A^{*}\right)$, the boundary of $f$ is the function $\partial f: V(G) \rightarrow A$ defined by

$$
\partial f=\sum_{h \in H_{G}(v)} \tau(h) f\left(e_{h}\right),
$$

where $e_{h}$ is the edge of $G$ containing $h$ and the summation is taken in $A$. If $\partial f=0$, then $(\tau, f)$ is an $A$-flow of $G$. In addition, $(\tau, f)$ is a nowhere-zero $A$-flow if both $f \in F\left(G, A^{*}\right)$ and $\partial f=0$. For any $f \in F\left(G, A^{*}\right)$, each positive edge contributes 0 , each sink edge $e$ contributes $2 f(e)$, and 
each source edge $e$ contributes $-2 f(e)$ to $\sum_{v \in V(G)} \partial f(v)$. Thus one has

$$
\sum_{v \in V(G)} \partial f(v)=\sum_{e \text { is a sink edge }} 2 f(e)-\sum_{e \text { is a source edge }} 2 f(e) \in 2 A .
$$

In [50], the authors introduced the definition of group connectivity of signed graphs. We extend this notation to a mod $k f$-weighted $b$-orientation (an $(f, b ; k)$-orientation) of signed graphs.

Let $(G, \sigma)$ be a 2 -unbalanced signed graph. A mapping $b: V(G) \rightarrow \mathbb{Z}_{k}$ is called an $\mathbb{Z}_{k^{-}}$ boundary of $(G, \sigma)$ if

$$
\sum_{v \in V(G)} b(v)=2 \alpha \text { for some } \alpha \in \mathbb{Z}_{k} .
$$

Let $Z\left(G, \mathbb{Z}_{k}\right)$ be the collection of all $\mathbb{Z}_{k}$-boundaries. Given a signed graph $(G, \sigma)$, for every $b \in Z\left(G, \mathbb{Z}_{k}\right)$ and every $f \in F\left(G, \mathbb{Z}_{k}^{*}\right)$, an orientation $\tau$ of $(G, \sigma)$ is an $(f, b ; k)$-orientation if for every vertex $v \in V(G)$,

$$
\partial f(v)=\sum_{h \in H_{G}(v)} \tau(v) f\left(e_{h}\right)=b(v) .
$$

As graphs are signed graphs with negativeness zero, it is again necessary to assume $k$ to be a prime when studying $(f, b ; k)$-orientations of signed graphs. Let $p>1$ be a prime. For notational simplification, we continue using $\mathcal{O}_{p}$ to denote the signed graph family $\mathcal{O}_{p}$ such that $(G, \sigma) \in \mathcal{O}_{p}$ if and only if $(G, \sigma)$ admits an $(f, b ; p)$-orientation for any $f \in F\left(G, \mathbb{Z}_{p}^{*}\right)$ and any $b \in Z\left(G, \mathbb{Z}_{k}\right)$. To avoid triviality, throughout the rest of this section, we always assume signed graphs under discussion with negativeness at least one.

Lemma 5.4.1. Weighted modulo orientability is invariant under the switch operation.

Proof. Let $(G, \sigma)$ be a 2-unbalanced signed graph. As every switching operation can be composed from the switching operations on trivial edge-cut, it suffices to verify this lemma for the switch operation $\zeta_{u}$ on the trivial edge-cut $S=E_{G}(u)$ for any given vertex $u$. We fix a vertex $u$ and let $\zeta=\zeta_{u}$ in the discussion below. Then $\sigma^{\prime}=\sigma \zeta$ is an signature equivalent to $\sigma$. We are to show that for any $f^{\prime} \in F\left(G, \mathbb{Z}_{k}^{*}\right)$ and any $b^{\prime} \in Z\left(G, \mathbb{Z}_{k}\right)$, the signed graph $\left(G, \sigma^{\prime}\right)$ also admits an $\left(f^{\prime}, b^{\prime} ; k\right)$-orientation.

Let $f=f^{\prime}$ and define $b: V(G) \rightarrow \mathbb{Z}_{k}$ by setting $b(u)=-b^{\prime}(u)$ and $b(v)=b^{\prime}(v)$ for any $v \in V(G) \backslash\{u\}$. As $b^{\prime} \in Z\left(G, \mathbb{Z}_{k}\right)$, we also have

$$
\sum_{v \in V(G)} b(v)=-b^{\prime}(u)+\sum_{v \in V(G) \backslash\{u\}} b^{\prime}(v)=\sum_{v \in V(G)} b^{\prime}(v)-2 b^{\prime}(u) \in 2 \mathbb{Z}_{k} .
$$

Thus $b \in Z\left(G, \mathbb{Z}_{k}\right)$ is also an $\mathbb{Z}_{k}$-boundary of $(G, \sigma)$. Since $(G, \sigma)$ admits an $(f, b ; k)$-orientation, there exists an orientation $\tau$ such that, for every vertex $v \in V(G)$,

$$
\partial f(v)=\sum_{h \in H_{G}(v)} \tau(h) f\left(e_{h}\right)=b(v) .
$$


Let $\tau^{\prime}$ be the orientation of $\left(G, \sigma^{\prime}\right)$ such that $\tau^{\prime}(h)=-\tau(h)$ if $h \in H_{G}(u)$ and $\tau^{\prime}(h)=\tau(h)$ otherwise. Hence, we have $\partial f^{\prime}(v)=\partial f(v)=\sum_{h \in H_{G}(v)} \tau^{\prime}(h) f\left(e_{h}\right)=b(v)=b^{\prime}(v)$ for any vertex $v \in V(G) \backslash\{u\}$. In addition,

$$
\partial f^{\prime}(u)=-\partial f(u)=\sum_{h \in H_{G}(u)} \tau^{\prime}(h) f\left(e_{h}\right)=\sum_{h \in H_{G}(u)}-\tau(h) f\left(e_{h}\right)=-b(u)=b^{\prime}(u) .
$$

Therefore, $\partial f^{\prime}=b^{\prime}$ in the signed graph $\left(G, \sigma^{\prime}\right)$ with orientation $\tau^{\prime}$.

Lemma 5.4.2. Let $K_{1}^{-t}$ be the graph obtained from $K_{1}$ by attaching $t$ negative loops to it. Then $K_{1}^{-t} \in \mathcal{O}_{p}$ if and only if $t \geq p-1$.

Proof. Let $V\left(K_{1}^{-t}\right)=\{v\}, H=t K_{2}$ be the signed graph with $V(H)=\left\{v, v^{\prime}\right\}$ such that there are $t$ positive edges joining $v$ and $v^{\prime}$. As $K_{1}^{-t}$ can be obtained from $H$ by identifying $v$ and $v^{\prime}$, we view that $E(H)=E\left(K_{1}^{-t}\right)$.

Assume first that $t \geq p-1$. Let $f \in F\left(K_{1}^{-t}, \mathbb{Z}_{p}^{*}\right)$ be an arbitrary mapping and $b(v) \in 2 \mathbb{Z}_{p}$ by an arbitrary $\mathbb{Z}_{p}$-boundary of $K_{1}^{-t}$. Since $b(v) \in 2 \mathbb{Z}_{p}$, there exists an element $\beta \in \mathbb{Z}_{p}$ such that $b(v)=2 \beta$. Define $b_{H} \in Z\left(H, \mathbb{Z}_{p}\right)$ by setting $b_{H}(v)=\beta$ and $b_{H}\left(v^{\prime}\right)=-\beta$. As $t \geq p-1$, by Lemma 5.1.3(vi), there exists an orientation $\tau$ of $H$ such that $\sum_{h \in H_{G}(v)} \tau(h) f\left(e_{h}\right)=\beta$ and $\sum_{h \in H_{G}\left(v^{\prime}\right)} \tau(h) f\left(e_{h}\right)=-\beta$. Since $K_{1}^{-t}$ can be obtained from $H$ by identifying $v$ and $v^{\prime}$, the orientation of $K_{1}^{-t}$ is obtained from $\tau$ of $H$ by taking the oppositive direction of every half edge in $H_{G}\left(v^{\prime}\right)$. Thus $K_{1}^{-t} \in \mathcal{O}_{p}$.

Conversely, we argue by contradiction and assume $K_{1}^{-t} \in \mathcal{O}_{p}$ but $t<p-1$. By Lemma 5.1.3(iv), there exists an element $\beta \in \mathbb{Z}_{p}$, a mapping $b^{\prime} \in Z\left(H, \mathbb{Z}_{p}\right)$ with $b^{\prime}(v)=\beta$ and $b^{\prime}\left(v^{\prime}\right)=-\beta$, and a mapping $f \in F\left(H, \mathbb{Z}_{p}^{*}\right)$ such that $H$ admits no $\left(f, b^{\prime} ; p\right)$-orientations. Let $b \in Z\left(K_{1}^{-t}, \mathbb{Z}_{p}\right)$ be the mapping with $b(v)=2 \beta$. As $f \in F\left(K_{1}^{-t}, \mathbb{Z}_{p}^{*}\right)$ also, if $K_{1}^{-t}$ has an $(f, b ; p)$ orientation $\tau^{\prime}$, then $\tau^{\prime}$ also give rise to an $\left(f, b^{\prime} ; p\right)$-orientation of $H$, contrary to the fact that $H$ admits no $\left(f, b^{\prime} ; p\right)$-orientations. This contradiction indicates that we must have $t \geq p-1$.

Thus we have the following observation immediately.

Observation 5.4.1. If $(G, \sigma) \in \mathcal{O}_{k}$ is an unbalanced signed graph, then $\epsilon_{N} \geq k-1$.

Lemma 5.4.3. Let $k$ be a positive integer and let $(H, \sigma)$ be a signed graph. Assume that either $E_{\sigma}^{-}(H)=\emptyset$ and $H \in \mathcal{O}_{k}$ is as an ordinary graph or $(H, \sigma) \in \mathcal{O}_{k}$ is as a $(k-1)$-unbalanced signed graph. If $\left(G, \sigma^{\prime}\right)$ is a $(k-1)$-unbalanced signed graph containing $(H, \sigma)$ as a subgraph, then $\left(G, \sigma^{\prime}\right) \in \mathcal{O}_{k}$ if and only if $\left(G / H, \sigma^{\prime \prime}\right) \in \mathcal{O}_{k}$.

Proof. The necessity follows from Lemma 5.1.3(ii). It remains to prove the sufficiency.

Let $f \in F\left(G, \mathbb{Z}_{k}^{*}\right)$ and $b \in Z\left(G, \mathbb{Z}_{k}\right)$ be given, and let $v_{H}$ be the vertex in $G / H$ onto which $H$ is contracted. For notational convenience, let $E_{\sigma}^{-}(H)$ denote the set of all negative edges of 
$(H, \sigma)$, as well as the set of negative loops incident with $v_{H}$ in $G / H$ obtained by contracting $H$. Let $f_{1} \in F\left(G / H, \mathbb{Z}_{k}^{*}\right)$ be the restriction of $f$ on $E(G / H)$, and define $b_{1}\left(v_{H}\right)=\sum_{v \in V(H)} b(v)$ and $b_{1}(v)=b(v)$ if $v \in V(G / H)-\left\{v_{H}\right\}$. Direct verification shows that $b_{1} \in Z\left(G / H, \mathbb{Z}_{k}\right)$. Since $G / H \in \mathcal{O}_{k}$, there exists an $\left(f_{1}, b_{1} ; p\right)$-orientation $\tau_{1}$ of $G / H$, and so $\partial f_{1}=b_{1}$.

For each vertex $v \in V(H)$, let $X_{1}(v)$ be the set of half edges incident with $v$ in $E(G)-E(H)$, and $X_{2}(v)$ be the set of half edges incident with $v$ in $E_{\sigma}^{-}(H)$. Define $b_{2}: V(H) \rightarrow \mathbb{Z}_{k}$ by

$$
b_{2}(v)=b(v)-\sum_{h \in X_{1}(v)} \tau(h) f_{1}\left(e_{h}\right)
$$

Since $\partial f_{1}=b_{1}$ in $G / H$, we have

$$
\sum_{v \in V(H)} \sum_{h \in X_{1}(v) \cup X_{2}(v)} \tau(h) f_{1}\left(e_{h}\right)=\partial f_{1}\left(v_{H}\right)=b_{1}\left(v_{H}\right)=\sum_{v \in V(H)} b(v) .
$$

By (6.5),

$$
\begin{aligned}
\sum_{v \in V(H)} b_{2}(v) & =\sum_{v \in V(H)} b(v)-\sum_{v \in V(H)} \sum_{h \in X_{1}(v)} \tau(h) f_{1}\left(e_{h}\right) \\
& =\sum_{v \in V(H)} \sum_{h \in X_{2}(v)} \tau(h) f_{1}\left(e_{h}\right)=\sum_{e \in E_{\sigma}^{-}(H)} \pm 2 f_{1}(e) \in 2 \mathbb{Z}_{k} .
\end{aligned}
$$

In the case when $E_{\sigma}^{-}(H)=\emptyset, b_{2}$ is a zero sum function, and so we always have $b_{2} \in Z\left(H, \mathbb{Z}_{k}\right)$. Let $f_{2} \in F\left(H, \mathbb{Z}_{k}^{*}\right)$ be the restriction of $f$ in $E(H)$. Since $H \in \mathcal{O}_{k}$, there exists an orientation $\tau_{2}$ of $H$ such that $\partial f_{2}=b_{2}$. Let $\tau=\tau_{1} \cup \tau_{2}$ be the orientation of $G$ formed by combing the orientation $\tau_{2}$ of $H$ and the orientation $\tau_{1}$ of $G / H$. Then, for each vertex $v \in V(H)$, it follows from (6.5) that

$$
\begin{aligned}
\partial f(v) & =\partial f_{1}(v)+\partial f_{2}(v) \\
& =\sum_{h \in X_{1}(v)} \tau(h) f_{1}\left(e_{h}\right)+b_{2}(v) \\
& =\sum_{h \in X_{1}(v)} \tau(h) f_{1}\left(e_{h}\right)+\left[b(v)-\sum_{h \in X_{1}(v)} \tau(h) f_{1}\left(e_{h}\right)\right]=b(v) .
\end{aligned}
$$

Therefore, $\tau$ is an $(f, b ; k)$-orientation of $\left(G, \sigma^{\prime}\right)$. By definition, $\left(G, \sigma^{\prime}\right) \in \mathcal{O}_{k}$.

Lemma 5.4.3 leads to a reduction method for verifying weighted modulo orientability of unbalanced signed graphs, which is an extension of Lemma 5.1.3(iii) for unsigned graphs. The following lemma follows Lemma 5.4.2 and Lemma 5.4.3.

Lemma 5.4.4. A unbalanced signed graph $(G, \sigma) \in \mathcal{O}_{p}$ if and only if it can be contracted to $K_{1}^{-t}$ for some integer $t \geq p-1$ by contracting its subgraphs in $\mathcal{O}_{p}$ recursively.

Lemma 5.4.5 below is a consequence by combining Lemma 5.4.3 and Lemma 5.4.4. 
Lemma 5.4.5. Let $(G, \sigma)$ be a $(p-1)$-unbalanced signed graph. If $G\left[E^{+}\right]$is spanning and $G\left[E^{+}\right] \in \mathcal{O}_{p}$ is as an ordinary graph, then $(G, \sigma) \in \mathcal{O}_{p}$.

The following theorems are our main results of this section.

Theorem 5.4.6. Let $p$ be an odd prime and let $(G, \sigma)$ be a $(p-1)$-unbalanced signed graph with $\kappa^{\prime}(G) \geq 12 p^{2}-28 p+15$. Then $(G, \sigma) \in \mathcal{O}_{p}$.

Proof. Given any $f \in F\left(G, \mathbb{Z}_{p}^{*}\right)$ and any $\mathbb{Z}_{p}$-boundary $b$. Since $p$ is prime, we have $2 \mathbb{Z}_{p}=\mathbb{Z}_{p}$ and $\sum_{v \in V(G)} b(v)$ can be any element in $\mathbb{Z}_{p}$. By Lemma 6.2.1, we may assume that $\left|E_{\sigma}^{-}(G)\right|=\epsilon_{N}$. Since $(G, \sigma)$ is a $\left(12 p^{2}-28 p+15\right)$-edge-connected signed graph with minimal number of negative edges in the switch equivalent class, $\left|S \cap E_{\sigma}^{-}(G)\right| \leq \frac{1}{2}|S|$ for each edge-cut $S$. Therefore $E_{\sigma}^{+}$is $\left(6 p^{2}-14 p+8\right)$-edge-connected and hence $G\left[E^{+}\right] \in \mathcal{O}_{p}$ by Theorem 1.2.9. By Lemma 5.4.5, one has $(G, \sigma) \in \mathcal{O}_{p}$.

Theorem 5.4.7. Let $p$ be an odd prime and let $(G, \sigma)$ be a $(p-1)$-unbalanced signed seriesparallel graph with $\kappa^{\prime}(G) \geq 4 p-7$. Then $(G, \sigma) \in \mathcal{O}_{p}$.

Proof. We prove by induction on $|V(G)|$. The statement clearly holds for $|V(G)|=1$ by Lemma 5.4.2. Assume $|V(G)| \geq 2$. The underlining simple graph $H$ of $G$ is $K_{4}$-minor-free, and so contains a vertex $v$ of degree at most 2. Denote $N_{H}(v)=\{x, y\}$ if $v$ has two neighbors and $N_{H}(v)=\{x\}$ if $v$ has a unique neighbor. In the signed graph $G$, by the edge connectivity $\kappa^{\prime}(G) \geq 4 p-7$, we have $\left|[v, x]_{G}\right|+\left|[v, y]_{G}\right| \geq 4 p-7$. Hence $\max \left\{\left|[v, x]_{G},\right|[v, y]_{G} \mid\right\} \geq 2 p-3$. We may, with out loss of generality, assume $\left|[v, x]_{G}\right| \geq 2 p-3$. (In the case $N_{H}(v)=\{x\}$, we have $\left|[v, x]_{G}\right| \geq 4 p-7 \geq 2 p-3$ as well.) By Lemma 6.2.1, by possible some switching operation at least half of edges in $[v, x]_{G}$ are positive, and so there are at least $p-1$ parallel positive edges, denoted by $H$, in $[v, x]_{G}$. Thus by Lemma $5.1 .3(\mathrm{iv})$, those parallel positive edges $H$ in $[v, x]_{G}$ is in $\mathcal{O}_{p}$. Moreover, $G / H \in \mathcal{O}_{p}$ by induction, and so $(G, \sigma) \in \mathcal{O}_{p}$ by Lemma 5.4.3. 


\section{Chapter 6}

\section{Almost all 9-Regular Graphs admit a Modulo 5-Orientation}

This chapter is a complete manuscript, but not yet published.

\subsection{Introduction}

We show that the assertion of Conjecture 1.2.4 holds asymptotically almost surely for random 9-regular graphs. It is known that a typical $(4 p+1)$-regular graph is $(4 p+1)$-edge connected. Alon and Prałat [7] showed that Jaeger's Circular Flow Conjecture holds for almost all $(4 p+1)$ regular graphs, provided that $p$ is large enough. Prałat and Wormald [68] showed that this conjecture with $p=1$ holds for almost all 5-regular graphs. It is thus natural to try and prove that this conjecture with $p=2$ holds for almost all 9-regular graphs.

Theorem 6.1.1. Almost all 9-regular graph $G$ on $n$ vertices admit modulo 5-orientations.

\subsection{Proof of Theorem 6.1.1}

The pairing model for investigating properties of random regular graphs was instigated by Bollobàs [3]. This consists of $d n$ points that are arranged in $n$ groups (called vertices) of $d$ each, arranged in pairs uniformly at random. The pairs induce a multigraph in the obvious way, and we refer to pairs as edges. This pairing model, called $\mathcal{P}_{n, d}$, is useful because simple graphs occur with equal probabilities, and the probability that it is simple for fixed $\mathrm{d}$ is bounded away from 0 . Hence, to show that the random regular graph has a property a.a.s.(asymptotically almost surely), it is enough to show that the random member of the multigraph corresponding to $\mathcal{P}_{n, d}$ a.a.s. has the same property or is non-simple. (See [12] for more information on this and other claims we make about $\left.\mathcal{P}_{n, d}.\right)$ We will work with orientations of (the pairs of) a pairing in $\mathcal{P}_{n, 9}$ 
in which each vertex has in-degree 2 or 7 . We call such orientations valid. Given an orientation, vertices of in-degree 2 will be called in-vertices, and those of out-degree 2 out-vertices, and each point contained in an edge oriented towards an in-vertex, or away from an out-vertex, is called special. Moreover, a point is an in-point if the edge containing it is oriented towards it, and an out-point otherwise.

Let $Y=Y(n)$ be the number of valid orientations of a random element of $\mathcal{P}_{n, 5}$. It is easy to see that

$$
\mathbb{E}[Y]=\frac{\left(\begin{array}{c}
n \\
n / 2
\end{array}\right)\left(\begin{array}{c}
9 \\
2
\end{array}\right)^{n}(9 n / 2) !}{M(9 n)},
$$

where

$$
M(s)=\frac{s !}{(s / 2) !\left(2^{s / 2}\right)}
$$

is the number of perfect matchings of $s$ points. Indeed, there are $\left(\begin{array}{c}n \\ n / 2\end{array}\right)$ ways to select in-vertices (since exactly half of the vertices must be such), $\left(\begin{array}{l}9 \\ 2\end{array}\right)^{n}$ ways to select two special points in each vertex, which determines each point to be either in or out, $(9 n / 2)$ ! ways to pair up the points so that each in is paired with an out and $M(9 n)$ pairings in total. Using Stirling's formula $s ! \sim \sqrt{2 \pi s}(s / e)^{s}$, we get

$$
\mathbb{E}[Y]=\frac{n ! \cdot 36^{n} \cdot(9 n / 2) !^{2} \cdot 2^{9 n / 2}}{(9 n) ! \cdot(n / 2) !^{2}} \sim 3 \cdot\left(\frac{81}{8}\right)^{n / 2} .
$$

This tells us that there are plenty of valid orientations per pairing, on average. To show that pairings a.a.s. have at least one valid orientation, i.e. that $\mathbb{P}(Y>0) \sim 1$, a common method would be to estimate $\mathbb{E}[Y(Y-1)]$, show that it is asymptotic to $(\mathbb{E}[Y])^{2}$, and then apply Chebyshev's inequality.

Lemma 6.2.1. (Second Moment Method) If $Y$ is a non-negative random variable and $\frac{\mathbb{E}\left[Y^{2}\right]}{\mathbb{E}[Y]^{2}} \rightarrow 0$ as $n \rightarrow \infty$, then a.s.s. $\mathbb{P}(Y=Y(n)>0) \rightarrow 1$.

First we estimate $\mathbb{E}[Y(Y-1)]$, considering any two orientations of the same 9-regular graph. Suppose that precisely $k$ vertices are in-vertices in both orientations, and that, of these, precisely $k_{20}$ have the same two special points and $k_{10}$ have the same exact one special point in both orientations. Since the first orientation induces $n / 2$ in-vertices, exactly $(n / 2-k)$ vertices are in-vertices in the first orientation but out-vertices in the second one. Of these, suppose that $k_{21}$ have the both two special points coinciding and $k_{11}$ have the exact one same special point. Similarly, there are $k$ vertices that are out-vertices in both orientations; suppose that $k_{22}$ of them have the both two special points coinciding and $k_{21}$ have exact one same special point. Finally, there are $(n / 2-k)$ vertices that are out-in the first and in-in the second orientation; suppose that $k_{23}$ of them have coinciding both two special points and $k_{13}$ have exact one same special point. Note that $k_{20}+k_{10} \leq k, k_{22}+k_{12} \leq k, k_{21}+k_{11} \leq n / 2-k$ and $k_{23}+k_{13} \leq n / 2-k$. It 
turns out that there are no additional restrictions on these parameters, other than integrality and non-negativity. We define

$$
\begin{aligned}
I= & \left\{\left(k, k_{10}, k_{20}, k_{11}, k_{21}, k_{12}, k_{22}, k_{13}, k_{23}\right) \in \mathbb{N}_{0}^{9}: k \leq \frac{n}{2}, \max \left\{k_{10}+k_{20}, k_{12}+k_{22}\right\} \leq k,\right. \\
& \left.\max \left\{k_{11}+k_{21}, k_{13}+k_{23}\right\} \leq \frac{n}{2}-k\right\},
\end{aligned}
$$

where $\mathbb{N}_{0}=\mathbb{N} \cup\{0\}$. Fix $\mathbf{k}=\left(k, k_{10}, k_{20}, k_{11}, k_{21}, k_{12}, k_{22}, k_{13}, k_{23}\right) \in I$. We next calculate the number of configurations, i.e. pairings with two given orientations, corresponding to this vector. Letting $n_{1}=k_{10} ! k_{20} ! k_{11} ! k_{21} ! k_{12} ! k_{22} ! k_{13} ! k_{23} !\left(k-k_{10}-k_{20}\right) !\left(\frac{n}{2}-k-k_{11}-k_{21}\right) !\left(k-k_{12}-k_{22}\right) !\left(\frac{n}{2}-\right.$ $\left.k-k_{13}-k_{23}\right)$ !, there are

$$
\frac{n !}{n_{1}}
$$

ways to partition the vertices into the twelve groups. There are then

$$
\begin{aligned}
& \left(\begin{array}{l}
9 \\
2
\end{array}\right)^{k_{20}+k_{21}+k_{22}+k_{23}} \cdot\left(\left(\begin{array}{l}
9 \\
1
\end{array}\right)\left(\begin{array}{l}
8 \\
1
\end{array}\right)\left(\begin{array}{l}
7 \\
1
\end{array}\right)\right)^{k_{10}+k_{11}+k_{12}+k_{13}} \\
& \cdot\left(\left(\begin{array}{l}
9 \\
2
\end{array}\right)\left(\begin{array}{l}
7 \\
2
\end{array}\right)\right)^{\left(k-k_{10}-k_{20}\right)+\left(\frac{n}{2}-k-k_{11}-k_{21}\right)+\left(k-k_{12}-k_{22}\right)+\left(\frac{n}{2}-k-k_{13}-k_{23}\right)}
\end{aligned}
$$

ways to assign special points in the two orientations, which is equal to

$$
36^{k_{20}+k_{21}+k_{22}+k_{23}} 504^{k_{10}+k_{11}+k_{12}+k_{13}} 756^{n-k_{10}-k_{20}-k_{11}-k_{21}-k_{12}-k_{22}-k_{13}-k_{23}} .
$$

Next, we need to pair (in,in)-points with (out,out)-points (where the first in refers to the first orientation, and so on). and (in,out)- with (out,in)-points. The number of (in,in)-points is equal to

$$
\begin{aligned}
& 2 k_{20}+k_{10}+k_{11}+7 k_{22}+6 k_{12}+k_{13}+5\left(k-k_{12}-k_{22}\right) \\
& +2\left(\frac{n}{2}-k-k_{11}-k_{21}\right)+2\left(\frac{n}{2}-k-k_{13}-k_{23}\right) \\
& =2 n+k+k_{10}+2 k_{20}-k_{11}-2 k_{21}+k_{12}+2 k_{22}-k_{13}-2 k_{23}
\end{aligned}
$$

and the same applies for (out,out). These two sets must be paired with each other. Half of the remaining points, or

$$
\frac{9 n}{2}-\left(2 n+k+k_{10}+2 k_{20}-k_{11}-2 k_{21}+k_{12}+2 k_{22}-k_{13}-2 k_{23}\right)
$$

will be in-out, and an equal number will be out-in. Hence, with $n_{2}=2 n+k+k_{10}+2 k_{20}-k_{11}-$ $2 k_{21}+k_{12}+2 k_{22}-k_{13}-2 k_{23}$, there are

$$
n_{2} !\left(\frac{9 n}{2}-n_{2}\right) !
$$

ways to legally pair the points. The number of configurations is the product of (6.2), (6.3) and (6.4). To obtain the expected number of pairs of orientations, we must divide by the number $M(9 n)$ of pairings. Putting $\mathbf{z}=\mathbf{z}(\mathbf{k})=\mathbf{k} / n$ and applying Stirling's formula again, we can write

$$
\mathbb{E}[Y(Y-1)]=\sum_{\mathbf{k} \in \mathbf{I}} r(\mathbf{z}) g(\mathbf{z}) \exp (n f(\mathbf{z}))
$$


where the various factors are defined as follows. The function $r$, which is the error factor in the applications of Stirling's formula, has the property that $r=O(1)$ for all $\mathbf{z}$, and $r \sim 1$ if $z$ is bounded away from the boundary of

$$
\begin{aligned}
J= & \left\{\left(z, z_{10}, z_{20}, z_{11}, z_{21}, z_{12}, z_{22}, z_{13}, z_{23}\right) \in \mathbb{R}_{0}^{9}: z \leq \frac{1}{2}, \max \left\{z_{10}+z_{20}, z_{12}+z_{22}\right\} \leq z\right. \\
& \left.\max \left\{z_{11}+z_{21}, z_{13}+z_{23}\right\} \leq \frac{1}{2}-z\right\}
\end{aligned}
$$

where $\mathbb{R}_{0}$ is the set of non-negative reals. With $b=z+z_{10}+2 z_{20}-z_{11}-2 z_{21}+z_{12}+2 z_{22}-z_{13}-2 z_{23}$, $c=z_{10} z_{20} z_{11} z_{21} z_{12} z_{22} z_{13} z_{23}\left(z-z_{10}-z_{20}\right)\left(1 / 2-z-z_{11}-z_{21}\right)\left(z-z_{12}-z_{22}\right)\left(1 / 2-z-z_{13}-z_{23}\right)$ and $h(x)=x \log x$, we have

$$
g=\frac{1}{48} \cdot \sqrt{\frac{(b+2)(5 / 2-b)}{2 c(\pi n)^{9}}}
$$

from the polynomial factors in Stirling's formula, and

$$
\begin{aligned}
f & =\left(z_{20}+z_{21}+z_{22}+z_{23}\right) \log 36+\left(z_{10}+z_{11}+z_{12}+z_{13}\right) \log 504+\frac{9}{2} \log 2 \\
& +\left(1-z_{10}-z_{20}-z_{11}-z_{21}-z_{12}-z_{22}-z_{13}-z_{23}\right) \log 756 \\
& +h(b+2)+h\left(\frac{5}{2}-b\right)+h\left(\frac{9}{2}\right)-h(9)-h\left(z_{10}\right)-h\left(z_{20}\right)-h\left(z_{11}\right) \\
& -h\left(z_{21}\right)-h\left(z_{12}\right)-h\left(z_{22}\right)-h\left(z_{13}\right)-h\left(z_{23}\right)-h\left(z-z_{10}-z_{20}\right) \\
& -h\left(1 / 2-z-z_{11}-z_{21}\right)-h\left(z-z_{12}-z_{22}\right)-h\left(1 / 2-z-z_{13}-z_{23}\right)
\end{aligned}
$$

from the rest.

Note that we can extend the definition of $f$ continuously to the boundary of $J$ by defining $x \log x=0$ at $x=0$. Then $f$ achieves its maximum on $J$. By Matlab (see Appendix), we determine the unique global maximum $f$ on $J$ at point

$$
\tilde{\mathbf{z}}=(0.1669,0.0596,0.0037,0.1402,0.0116,0.1572,0.0097,0.1402,0.0116) .
$$

Next part of the proof consists of a routine computation and argument. Set $\mathbf{y}=\mathbf{z}-\tilde{\mathbf{z}}$. Then expand $f(\mathbf{z})$ about the global maximum point $\tilde{\mathbf{z}}$, to obtain

$$
f=2.122+A^{T} \mathbf{y}+\frac{1}{2} \mathbf{y}^{T} B \mathbf{y}+O\left(x^{3}\right),
$$

where $y^{T}$ denotes the transpose of $y$, and

$$
\begin{gathered}
A \sim(-39.0936,0,0,0,0,39.0936,39.0936,0,0), \\
B \sim\left(B_{1} \mid B_{2}\right)
\end{gathered}
$$


with

$$
B_{1}=\left(\begin{array}{ccccc}
1.9215 \times 10^{17} & 10.5424 & 11.4362 & -6.4092 & -7.3029 \\
10.5424 & -25.5403 & -7.8610 & -0.8938 & -1.7876 \\
11.4362 & -7.8610 & -278.6243 & -1.7876 & -3.5751 \\
-6.4092 & -0.8938 & -1.7876 & -11.7548 & -3.7278 \\
-7.3029 & -1.7876 & -3.5751 & -3.7278 & -88.0466 \\
-1.9215 \times 10^{17} & 0.8938 & 1.7876 & -0.8938 & -1.7876 \\
-5.7646 \times 10^{17} & 1.7876 & 3.5751 & -1.7876 & -3.5751 \\
-6.4092 & -0.8938 & -1.7876 & 0.8938 & 1.7876 \\
-7.3029 & -1.7876 & -3.5751 & 1.7876 & 3.5751
\end{array}\right)
$$

and

$$
B_{2}=\left(\begin{array}{cccc}
-1.9215 \times 10^{17} & -1.9215 \times 10^{17} & -6.4092 & -7.303 \\
0.8938 & 1.7876 & -0.8938 & -1.7876 \\
1.7876 & 3.5751 & -1.7876 & -3.5751 \\
-0.8938 & -1.7876 & 0.8938 & 1.7876 \\
-1.7876 & -3.5751 & 1.7876 & 3.5751 \\
1.9215 \times 10^{17} & 1.9215 \times 10^{17} & -0.8938 & -1.7876 \\
1.9215 \times 10^{17} & 1.9215 \times 10^{17} & -1.7876 & -3.5751 \\
-0.8938 & -1.7876 & -11.7548 & -3.7278 \\
-1.7876 & -3.5751 & -3.7278 & -88.0467
\end{array}\right)
$$

and $x=x(\mathbf{z})=\|\mathbf{y}\|$, with $\|\cdot\|$ denoting the $L^{2}$ norm (say). The error term in this expansion is valid by Taylor's theorem provided that $x=o(1)$.

Let $J_{0}:=\left\{\mathbf{z}: x=o\left(n^{-2 / 9}\right)\right\}$. For $\mathbf{z} \in J_{0}$, we have $r(\mathbf{z}) g(\mathbf{z}) \sim g(\tilde{\mathbf{z}})$ and $x^{3}=o(n-6 / 9)$. Thus

$$
\sum_{\mathbf{k} / \mathbf{n} \in \mathbf{J}_{\mathbf{0}}} r(\mathbf{z}) g(\mathbf{z}) \exp (n f(\mathbf{z})) \sim \exp (2.122 n) g(\tilde{\mathbf{z}}) \sum_{\mathbf{k} / \mathbf{n} \in \mathbf{J}_{\mathbf{0}}} \exp \left(n \mathbf{y}^{T} B \mathbf{y}\right) .
$$

The eigenvalues of $B$ are

$$
-2.7369,-88.4123,-64.6932,-34.7083,-22.4605,-15.8890,10.5265,53.4065,5.7646 \times 10^{17} .
$$

Thus

$$
\sum_{\mathbf{k} / \mathbf{n} \in \mathbf{J}_{\mathbf{0}}} \exp \left(n \mathbf{y}^{T} B \mathbf{y}\right) \sim n^{9} \int_{J_{0}} \exp \left(n \mathbf{y}^{T} B \mathbf{y}\right) d^{n} \mathbf{y}
$$

and

$$
\int_{J_{0}} \exp \left(n \mathbf{y}^{T} B \mathbf{y}\right) d^{n} \mathbf{y} \sim \int_{\mathbb{R}^{9}} \exp \left(n \mathbf{y}^{T} B \mathbf{y}\right) d^{n} \mathbf{y}=\frac{\pi^{9 / 2}}{n^{9 / 2} \sqrt{|\operatorname{det} B|}} .
$$

A simple computing allows us to estimate the summation in Eq. (6.5), and we obtain

$$
\mathbb{E}[Y(Y-1)] \sim \exp (2.122 n) \frac{g(\tilde{\mathbf{z}})(\pi n)^{9 / 2}}{\sqrt{|\operatorname{det} B|}} .
$$


We find that $g(\tilde{\mathbf{z}})=\frac{1.7944 \times 10^{16}}{48 \sqrt{2}(\pi n)^{9 / 2}}$ and $\sqrt{|\operatorname{det} B|}=1.7895 \times 10^{15}$. So

$$
\mathbb{E}[Y(Y-1)] \sim \exp (2.122 n) \cdot \frac{1.7944 \times 10^{16}}{1.2146 \times 10^{17}} \sim \frac{\exp (2.122 n)}{12} .
$$

Combining this with Eq. (6.1), we have

$$
\frac{\mathbb{E}\left[Y^{2}\right]}{\mathbb{E}[Y]^{2}} \sim \frac{1}{108} \cdot\left(\frac{8 \exp (2.122)}{81}\right)^{n} \rightarrow 0 .
$$

Hence one can have $\mathbb{P}(Y=Y(n)>0) \rightarrow 1$ as $n \rightarrow \infty$, that is, almost all 9-regular graphs admit modulo 5-orientations.

Appendix

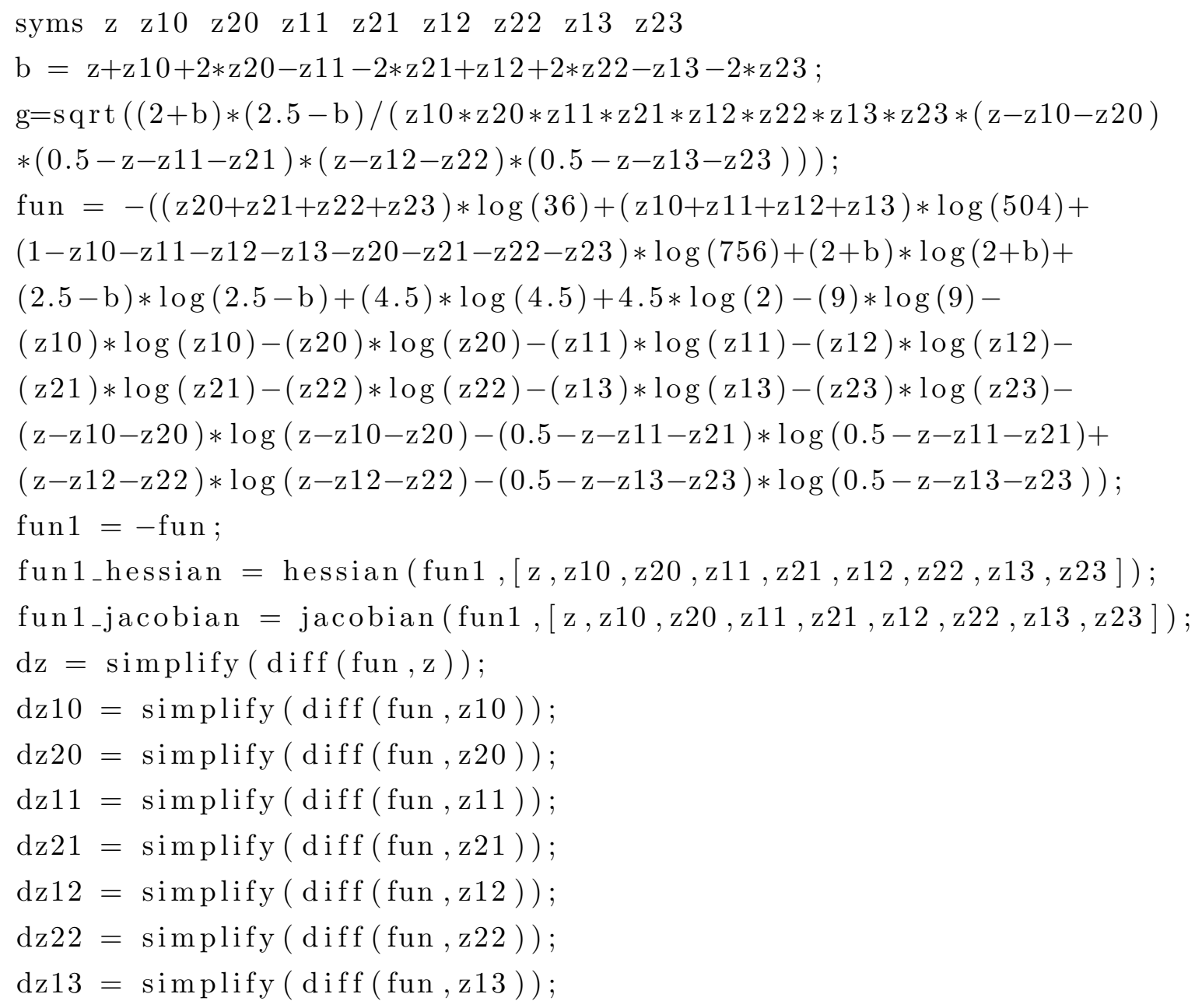




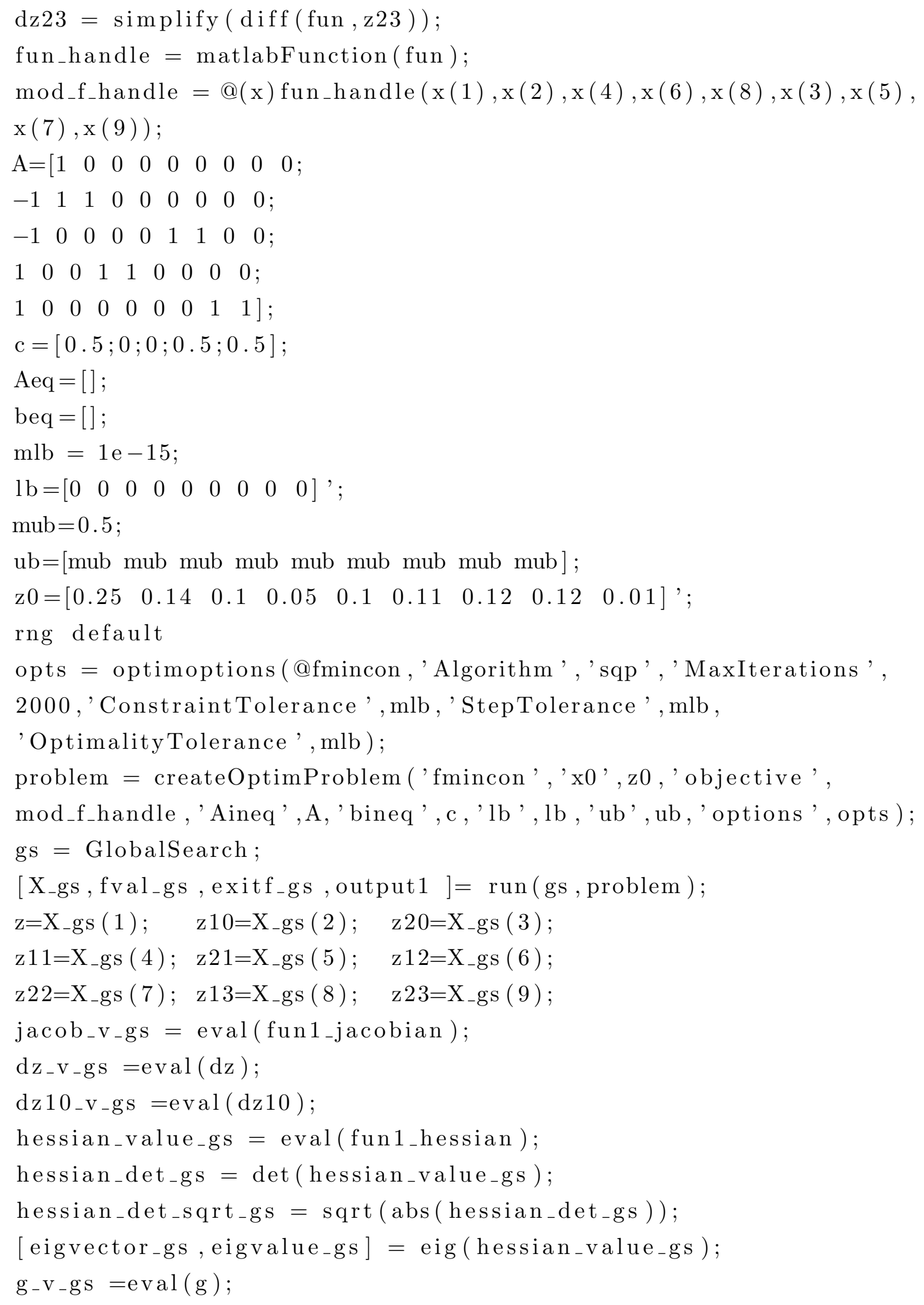




\section{Chapter 7}

\section{Final Remarks}

We conclude this dissertation with some future research problems on the flow of graphs.

\subsection{Validity of flow conjectures on Cayley graphs}

Let $\Gamma$ be a finite group. The Cayley graph on $\Gamma$ with a generating set $S$ of $\Gamma$, denoted by $\operatorname{Cay}(\Gamma, S)$, is the undirected graph with vertex set $\Gamma$ and edge set containing an edge joining $g$ to $g s$ whenever $g \in \Gamma$ and $s \in S$. Many researchers verified the validity of Tutte's 3-flow conjecture on Cayley graphs of certain classes of finite groups.

Theorem 7.1.1. (Alspach, Liu and Zhang, [6]) Every Cayley graph on a finite solvable group admits a nowhere-zero 4-flow. In particular, every cubic Cayley graph on a solvable group is 3-edge-colorable.

Theorem 7.1.2. (Potočnik, Škoviera and Škrekovski, [67]) Every Cayley graph of an abelian group and of valence at least 4 admits a Nowhere-zero 3-flow.

Theorem 7.1.3. (Yang,Li, [81]) Every Cayley graph of a dihedral group and of valence at least 4 admits a Nowhere-zero 3 -flow.

Theorem 7.1.4. (Li, [54]) Every Cayley graph of valence at least 4 on a generalized dihedral (or quaternion) group admits a Nowhere-zero 3-flow.

For other results, see $[3,53,54,65,66,81]$. In addition, Li et al. verified the validity of Conjecture 1.2.3 (2) on Cayley graphs on abelian groups.

Theorem 7.1.5. (Li et al., [58]) Every connected Cayley graph of degree at least 5 on an Abelian group is $\mathbb{Z}_{3}$-connected.

Based on above known results. The following problem is in our consideration. 
Problem 1. Verify the validity of Conjectures 1.2.2, 1.2.3, 1.2.4 and 1.2.5 on Cayley graphs.

In 1983, Bouchet [11] proposed the following conjecture.

Conjecture 7.1.1. (Bouchet [11]) Every flow-admissible signed graph admits a nowhere-zero 6-flow.

Similarly, we considered the following problem.

Problem 2. Verify the validity of Conjecture 7.1.1 on signed Cayley graphs.

\subsection{Weighted modulo orientation of graphs}

In this dissertation, we reduced the edge-connectivity $\left(6 p^{2}-14 p+8\right)$ in Theorem 1.2.9 for some graph families, and we extend the $(f, b ; p)$-orientation framework to signed graph. Viewing the results in this paper and in literatures, we believe that it is possible that a linear function of $p$ would suffice for such $(f, b ; p)$-orientations. We conclude the following conjectures.

Conjecture 7.2.1. There exists a constant c independent of p such that every cp-edge-connected graph is in $\mathcal{O}_{p}$.

Conjecture 7.2.2. There exists a constant $c$ independent of $p$ such that every cp-edge-connected $(p-1)$-unbalanced signed graph is in $\mathcal{O}_{p}$. 


\section{Bibliography}

[1] K. Appel and W. Haken, Every Planar Map is Four Colorable, Part I: Discharging, Illinois J. Math. 21 (1977), 429-490.

[2] K. Appel, W. Haken and J. Koch, Every Planar Map is Four Colorable, Part II: Reducibility, Illinois J. Math., 21 (1977), 491-567.

[3] M. Ahanjideh, A. Iranmanesh, The validity of Tutte's 3-flow conjecture for some Cayley graphs, ARS Math. Contemp., 16 (2019), 203-213.

[4] N. Alon, N. Linial and R. Meshulam, Additive bases of vector spaces over prime fields, J. Combin. Theory Ser. A, 57(1991), 203-210.

[5] N. Alon, M. Nathanson and I. Ruzsa, The polynomial method and restricted sums of congruence classes, J. Number Theory, 56(2) (1996), 404-417.

[6] B. Alspach, Y. Liu and C. Zhang, Nowhere-zero 4-flows and Cayley graphs on solvable groups, SIAM J. Discrete Math., 9 (1996), 151-154.

[7] N. Alon and P. Prałat, Modular orientations of random and quasi-random regular graphs, Combinatorics, Probability and Computing 20 (2011), 321-329.

[8] J.A. Bondy and U.S.R. Murty, Graph theory, Springer, New York, 2008.

[9] B. Bollobás, A probabilistic proof of an asymptotic formula for the number of labelled regular graphs, European J. Combin., 1 (1980), 311-316.

[10] F. Boesch and F. Harary, Line removal algorithms for graphs and their degree lists, IEEE Transactions on Circuits and Systems, 23 (1976), 778-782.

[11] A. Bouchet, Nowhere-zero integral flows on a bidirected graph, J. Combin. Theory Ser. B, 34(1983), 279-292.

[12] A.E. Brouwer and H.J. Veldman, Contractibility and NP-completeness, J. Graph Theory, 11 (1987), 71-79. 
[13] P.A. Catlin, The reduction of graph families closed under contraction, Discrete Math., 160 (1996), 67-80.

[14] P.A. Catlin, A. M. Hobbs and H.-J. Lai, Graph families operations, Discrete Math., 230 (2001), 71-97.

[15] A.L. Cauchy, Recherches sur les nombres, J. École Polytech., 9 (1813), 99-123.

[16] J. Chen, E. Eschen and H.-J. Lai, Group connectivity of certain graphs, Ars Combin., 89 (2008), 141-158.

[17] H. Davenport, On the addition of residue classes, J. London Math. Soc., 10 (1935), 30-32.

[18] X. Dai and J. Yin, A complete characterization of graphic sequences with a $\mathbb{Z}_{3}$-connected realization, European J. Combin., 51 (2016), 215-221.

[19] M. DeVos, R. Xu and G. Yu, Nowhere-zero $\mathbb{Z}_{3}$-flows through $\mathbb{Z}_{3}$-connectivity, Discrete Math., 306 (2006), 26-30.

[20] M. Delcourt and L. Postle, Progress towards Nash-Williams' Conjecture on Triangle Decompositions, arXiv:1909.00514.

[21] G.A. Dirac, A property of 4-chromatic graphs and some remarks on critical graphs, J. Lond. Math. Soc., 27 (1952), 85-92.

[22] K. Edwards, D. P. Sanders, P.D. Seymour and R. Thomas, Three-edge-colouring doublecross cubic graphs, J. Combin. Theory Ser. B, 119 (2016) 66-95.

[23] L. Esperet, R.J. De Verclos, T.N. Le and S. Thomassé, Additive Bases and Flows in Graphs, SIAM J. Discrete Math., 32(1) (2018), 534-542.

[24] L. Esperet, M. Montassier, P. Ochem and A. Pinlou, A Complexity Dichotomy for the Coloring of Sparse Graphs, J. Graph Theory, 73 (2013), 85-102.

[25] G. Fan and C. Zhou, Ore condition and nowhere-zero 3-flows, SIAM J. Discrete Math., 22 (2008), 288-294.

[26] W. Feller, An Introduction to Probability Theory and Its Applications Vol. 1 (3rd Edition), John Wiley \& Sons, Inc., 1968.

[27] B. Grübaum, Grötzsch's theorem on 3-colorings, Mich. Math. J., 10 (1963), 303-310.

[28] J.L. Gross and T.W. Tucker, Topological Graph Theory, Wiley-Interscience, New York, 1987. 
[29] S.L. Hakimi, On the degrees of the vertices of a directed graph, J. Franklin Inst., 279 (1965), 290-308.

[30] S.L. Hakimi, On the Realizability of a Set of Integers as Degrees of the Vertices of a Graph, SIAM J. Appl. Math., 10 (1962), 496-506.

[31] M. Han, J. Li, Y. Wu and C.-Q. Zhang. Counterexamples to Jaeger's circular flow conjecture, J. Combin. Theory, Ser. B, 131 (2018), 1-11.

[32] M. Han, H.-J. Lai and J.-B. Liu, Modulo 5-Orientations and Degree Sequences, Discrete Appl. Math., 260 (2019), 155-163.

[33] F. Jaeger, Flows and generalized coloring theorems in graphs, J. Combin. Theory Ser. B, 26 (1979), 205-216.

[34] F. Jaeger, On circular flows in graphs, in: Finite and Infinite Sets (Eger, 1981), in: Colloq. Math. Soc. Janos Bolyai, vol. 37, North-Holland, Amsterdam, 1984, 391-402.

[35] F. Jaeger, Nowhere-zero flow problems, in: Selected Topics in Graph Theory, vol. 3, L. Beineke and R. Wilson, eds., Academic Press, London, New York, 1988, 91-95.

[36] F. Jaeger, N. Linial, C. Payan and M. Tarsi, Group connectivity of graphs-a nonhomogeneous analogue of nowhere-zero flow properties, J. Combin. Theory Ser. B, 56(1992), 165-182.

[37] P.A. Kilpatrick, Tutte's First Colour-Cycle Conjecture, Ph.D. thesis, Cape Town, 1975.

[38] T. Kloks, Treewidth, Computations and Approximations, Springer-Verlag, New York, 1994.

[39] M. Kochol, Superposition and constructions of graphs without nowhere-zero $k$-flows, European J. Combin., 23 (2002), 281-306.

[40] M. Kamiński, D. Paulusma and D.M. Thilikos, Contractions of planar graphs in polynomial time, In: "Proceedings of ESA 2010", Lecture Notes in Computer Science 6346(2010), 122133.

[41] H.-J. Lai, Group Connectivity of 3-Edge-Connected Chordal Graphs, Graphs Combin., 16 (2000), 165-176.

[42] H.-J. Lai, Mod $(2 p+1)$-orientations and $K_{1,2 p+1}$-decompositions, SIAM J. Discrete Math., 21 (2007), 844-850.

[43] H.-J. Lai and H. Lai, Duality of graph families, Discrete Math., 110 (1992), 165-177. 
[44] H.-J. Lai, Y. Liang, J. Liu, Z. Miao, J. Meng, Y. Shao and Z. Zhang, On Strongly $\mathbb{Z}_{2 s+1^{-}}$ connected Graphs, Discrete Appl. Math., 174 (2014), 73-80.

[45] H.-J. Lai, X. Li , Y. H. Shao and M. Zhan, Group Connectivity and Group Colorings of Graphs-A survey, Acta Math. Sinica, English Series, 27 (2011), 405-434.

[46] H.-J. Lai, Y. Shao, H. Wu and J. Zhou, On mod $(2 p+1)$-orientations of graphs, J. Combin. Theory, Ser. B, 99 (2009), 399-406.

[47] J. Li, H.-J. Lai and R. Luo, Group Connectivity, Strongly $\mathbb{Z}_{m}$-Connectivity and Edge Disjoint Spanning Trees, SIAM J. Discrete Math., 31 (2017), 1909-1922.

[48] J. Li, Group Connectivity and Modulo Orientations of Graphs, PhD Dissertation, West Virginia University, 2018.

[49] J. Li, H.-J. Lai and R. Luo, Group Connectivity, Strongly $\mathbb{Z}_{m}$-Connectivity, and Edge Disjoint Spanning Trees, SIAM J. Discrete Math. 31(3) (2017), 1909-1922.

[50] J. Li, R. Luo, H. Ma and C.-Q. Zhang, Flow-contractible configurations and group connectivity of signed graphs, Discrete Math., 341(2018), 3227-3236.

[51] J. Li, R. Luo and Y. Wang, Nowhere-zero 3-flow of graphs with small independence number, Discrete Math., 341(1) (2018), 42-50.

[52] A. Levin, D. Paulusma and G.J. Woeginger, The computational complexity of graph contractions I: Polynomially solvable and NP-complete cases, Networks, 51 (2008), 178-189.

[53] X. Li and S. Zhou, Nowhere-zero 3-flows in graphs admitting solvable arc-transitive groups of automorphisms, arXiv preprint arXiv:1310.5317 (2013).

[54] L. Li and X. Li, Nowhere-zero 3-flows in Cayley graphs on generalized dihedral group and generalized quaternion group, Front. Math. China, 10 (2015), 293-302.

[55] J.-B. Liu, M. Han, H.-J. Lai and J. Li, Modulo Orientations and Matchings in Graphs, submitted.

[56] J.-B. Liu, P. Li, J. Li and H.-J. Lai, On weighted modulo orientation of graphs, Europ. J. Combinatorics, to be appeared.

[57] J.-B. Liu, M. Han and H.-J. Lai, Weighted modulo orientations of graphs and signed graphs, submitted.

[58] H. Li, P. Li, M. Zhan, T. Zhang and J. Zhou, $\mathbb{Z}_{3}$-connectivity in Abelian Cayley graphs, Discrete Math., 313(16) (2013), 1666-1676. 
[59] H.-J. Lai and C.-Q. Zhang, Nowhere-zero 3-flows of highly connected graphs, Discrete Math., 110 (1992), 179-183.

[60] L.M. Lovasz, C. Thomassen, Y. Wu and C.-Q. Zhang, Nowhere-zero 3-flows and modulo $k$-orientations, J. Combin. Theory, Ser. B, 103 (2013), 587-598.

[61] R. Luo, R. Xu, W. Zang and C.-Q. Zhang, Realizing degree sequences with graphs having nowhere-zero 3-flows, SIAM J. Discrete Math., 22 (2008), 500-519.

[62] R. Luo, W. Zang and C.-Q. Zhang, Nowhere-zero 4-flows, simultaneous edge-colorings and critical partial Latin squares, Combinatorica, 24 (2004), 641-657.

[63] H.B. Mann and Y.F. Wou, An addition theorem for the elementary abelian group of type ( $p, p)$, Monatsh Math., 102 (1986), 273-308.

[64] G. MacGillivray and M.H. Siggers, On the complexity of $H$-colouring planar graphs, Discrete Math., 309(18) (2009), 5729-5738.

[65] R. Nedela and M. Škoviera, Cayley snarks and almost simple groups, Combinatorica, 21 (2001), 583-590.

[66] M. Nanásiová and M. Śkoviera, Nowhere-zero 3-flows in Cayley graphs and Sylow 2subgroups, J. Algebraic Combin., 30 (2009), 103-111.

[67] P. Potočnik, M. Škoviera and R. Škrekovski, Nowhere-zero 3-flows in Abelian Cayley Graphs, Discrete Math., 297 (2005), 119-127.

[68] P. Prałat, N. Wormald. Almost all 5-regular graphs have a 3-flow. J. Graph Theory, 93(2) (2020), 147-56.

[69] N. Robertson and P.D. Seymour, Graph minors. XIII. The disjoint paths problem, J. Combin. Theory Ser. B, 63 (1995), 65-110.

[70] N. Robertson, D.P. Sanders, P.D Seymour and R. Thomas, The Four-Colour Theorem, J. Combin. Theory Ser. B, 70 (1997), 2-44.

[71] N. Robertson, P.D. Seymour and R. Thomas, Tutte's edge-coloring conjecture, J. Combin. Theory Ser. B, 70 (1997), 166-183.

[72] N. Robertson, P.D. Seymour and R. Thomas, Excluded minors in cubic graphs, arXiv:1403.2118.

[73] N. Robertson, P.D. Seymour and R. Thomas, Cyclically five-connected cubic graphs, J. Combin. Theory Ser. B, 125 (2017), 132-167. 
[74] P.D. Seymour, Tutte's three-edge-coloring conjecture, Proceedings of Graph Theory@ Georgia Tech, a conference honouring the 50th birhday of Robin Thomas, May 2012, https://smartech.gatech.edu/handle/1853/44224.

[75] R. Steinberg and D.H. Younger, Grötzsch's theorem for the projective plane, Ars Comb., 28 (1989), 15-31.

[76] P.D. Seymour, Nowhere-zero 6-flows, J. Combin. Theory Ser. B., 30(1981) 130-136.

[77] C. Thomassen, The weak 3-Flow Conjecture and the weak circular flow conjecture, J. Combin. Theory Ser. B, 102 (2012), 521-529.

[78] W.T. Tutte, On the embedding of linear graphs in surfaces, Proc. London Math. Soc. Ser., $2(51)(1949), 474-483$.

[79] W.T. Tutte, A contribution to the theory of chromatical polynomials, Can. J. Math., 6 (1954), 80-91.

[80] W.T. Tutte, On the algebraic theory of graph colourings, J. Combin. Theory, 1 (1966) $15-50$.

[81] F. Yang and X. Li, Nowhere-zero 3-flows in dihedral Cayley graphs, Inform. Process. Lett., 111 (2011), 416-419.

[82] N.C. Wormald, Models of random regular graphs, Surveys in Combinatorics, 1999, J.D. Lamb and D. A. Preece, eds. London Mathematical Society Lecture Note Series, vol 276, pp. 239 — 298, Cambridge University Press, Cambridge, 1999.

[83] Y. Wu, R. Luo, D. Ye and C.-Q. Zhang, A note on an extremal problem for group connectivity, European J. Combin., 40 (2014), 137-141.

[84] F. Yang, X. Li and H.-J. Lai, Realizing degree sequences as $\mathbb{Z}_{3}$-connected graphs, Discrete Math., 333 (2014), 110-119.

[85] W.T. Tutte, On the embedding of linear graphs in surfaces, Proc. London Math. Soc. Ser., $2(51)(1949), 474-483$.

[86] W.T. Tutte, A contribution to the theory of chromatical polynomials, Can. J. Math., 6(1954), 80-91.

[87] C.-Q. Zhang, Integer Flows and Cycle Covers of Graphs, Marcel Dekker Inc., New York, ISBN: 0-8247-9790-6, 1997. 